\title{
Do methodological choices in environmental modeling bias rebound effects? A case study on electric cars
}

\author{
David Font Vivanco, Arnold Tukker and René Kemp
}

Supporting information S1: Complete life cycle physical inventory for the full-battery electric, hydrogen fuel cell and internal combustion passenger cars

Supporting information S2: Total cost of ownership calculations

Supporting information S3: Model description, background data and results for the AIDS model

Supporting information S4: Results for the attributional life cycle assessment

Supporting information S5: Method for input-output-based hybrid life cycle assessment

Supporting information S6: Short description of the E3IOT, WIOD and Exiobase databases

Supporting information S7: Results of the two-way ANOVA test

Number of pages: 56

Number of figures: 0

Number of tables: 18 
1 Supporting information S1: Complete life cycle physical inventory for the full-battery electric,

2 hydrogen fuel cell and internal combustion passenger cars

Table S1.1 presents the complete physical life cycle inventory (production, use and end-of-life) for the full-battery electric, hydrogen fuel cell and internal combustion engine passenger car technologies.

Table S1.1. Physical inventory for the full-battery electric (FBE), hydrogen fuel cell (HFC) and internal combustion engine (ICE) passenger car technologies. Sources: Hawkins et al. ${ }^{1}$ and Bartolozzi, et al. ${ }^{2}$.

\begin{tabular}{|c|c|c|c|c|}
\hline Technology & Stage & Physical flow (as in Ecoinvent 2.2) & Value & Unit \\
\hline FBE & Construction & milling, steel, average[RER] & 56.5 & $\mathrm{~kg}$ \\
\hline FBE & Construction & milling, steel, average[RER] & 2.43 & $\mathrm{~kg}$ \\
\hline FBE & Construction & copper, primary, at refinery[ID] & 46.7 & $\mathrm{~kg}$ \\
\hline FBE & Construction & injection moulding[RER] & 43.5 & $\mathrm{~kg}$ \\
\hline FBE & Construction & $\begin{array}{l}\text { aluminium, production mix, wrought alloy, at } \\
\text { plant[RER] }\end{array}$ & 92.2 & $\mathrm{~kg}$ \\
\hline FBE & Construction & $\begin{array}{l}\text { aluminium product manufacturing, average metal } \\
\text { working[RER] }\end{array}$ & 92.2 & $\mathrm{~kg}$ \\
\hline FBE & Construction & milling, steel, average[RER] & 1.27 & $\mathrm{~kg}$ \\
\hline FBE & Construction & synthetic rubber, at plant[RER] & 30.5 & $\mathrm{~kg}$ \\
\hline FBE & Construction & polyester resin, unsaturated, at plant[RER] & 3.7 & $\mathrm{~kg}$ \\
\hline FBE & Construction & steel, converter, low-alloyed, at plant[RER] & 74.9 & $\mathrm{~kg}$ \\
\hline FBE & Construction & injection moulding[RER] & 12.6 & $\mathrm{~kg}$ \\
\hline FBE & Construction & $\begin{array}{l}\text { steel product manufacturing, average metal } \\
\text { working[RER] }\end{array}$ & 67.8 & $\mathrm{~kg}$ \\
\hline FBE & Construction & milling, steel, average[RER] & 0.51 & $\mathrm{~kg}$ \\
\hline FBE & Construction & $\begin{array}{l}\text { steel product manufacturing, average metal } \\
\text { working[RER] }\end{array}$ & 63.4 & $\mathrm{~kg}$ \\
\hline FBE & Construction & wire drawing, copper[RER] & 54.9 & $\mathrm{~kg}$ \\
\hline FBE & Construction & tempering, flat glass[RER] & 15.2 & $\mathrm{~kg}$ \\
\hline FBE & Construction & flat glass, uncoated, at plant[RER] & 15.2 & $\mathrm{~kg}$ \\
\hline FBE & Construction & copper, secondary, at refinery[RER] & 8.23 & $\mathrm{~kg}$ \\
\hline FBE & Construction & electricity, medium voltage, at grid $[\mathrm{CH}]$ & 443 & $\mathrm{kWh}$ \\
\hline FBE & Construction & water, completely softened, at plant[RER] & 14.7 & $\mathrm{~kg}$ \\
\hline FBE & Construction & $\begin{array}{l}\text { polyethylene terephthalate, granulate, bottle grade, at } \\
\text { plant[RER] }\end{array}$ & 25 & $\mathrm{~kg}$ \\
\hline FBE & Construction & steel, electric, un- and low-alloyed, at plant[RER] & 51 & $\mathrm{~kg}$ \\
\hline FBE & Construction & steel, converter, low-alloyed, at plant[RER] & 31.7 & $\mathrm{~kg}$ \\
\hline FBE & Construction & zinc coating, pieces[RER] & 192 & $\mathrm{~m} 2$ \\
\hline FBE & Construction & copper, primary, at refinery[ID] & 4.03 & $\mathrm{~kg}$ \\
\hline FBE & Construction & $\begin{array}{l}\text { steel product manufacturing, average metal } \\
\text { working[RER] }\end{array}$ & 23.6 & $\mathrm{~kg}$ \\
\hline FBE & Construction & alkyd paint, white, $60 \%$ in solvent, at plant[RER] & 7.86 & $\mathrm{~kg}$ \\
\hline FBE & Construction & milling, cast iron, average[RER] & 0.17 & $\mathrm{~kg}$ \\
\hline FBE & Construction & synthetic rubber, at plant[RER] & 5.66 & $\mathrm{~kg}$ \\
\hline FBE & Construction & hot rolling, steel[RER] & 20.2 & $\mathrm{~kg}$ \\
\hline FBE & Construction & steel, electric, un- and low-alloyed, at plant[RER] & 31.7 & $\mathrm{~kg}$ \\
\hline
\end{tabular}




\begin{tabular}{|c|c|c|c|c|}
\hline FBE & Construction & $\begin{array}{l}\text { steel product manufacturing, average metal } \\
\text { working[RER] }\end{array}$ & 18 & $\mathrm{~kg}$ \\
\hline FBE & Construction & steel, converter, low-alloyed, at plant[RER] & 15.9 & $\mathrm{~kg}$ \\
\hline FBE & Construction & $\begin{array}{l}\text { steel product manufacturing, average metal } \\
\text { working[RER] }\end{array}$ & 15.9 & $\mathrm{~kg}$ \\
\hline FBE & Construction & ferrite, at plant $[\mathrm{GLO}]$ & 4.52 & $\mathrm{~kg}$ \\
\hline FBE & Construction & powder coating, steel[RER] & 3.23 & $\mathrm{~m} 2$ \\
\hline FBE & Construction & injection moulding[RER] & 2.5 & $\mathrm{~kg}$ \\
\hline FBE & Construction & electricity, medium voltage, at grid $[\mathrm{CH}]$ & 146 & $\mathrm{kWh}$ \\
\hline FBE & Construction & $\begin{array}{l}\text { steel product manufacturing, average metal } \\
\text { working[RER] }\end{array}$ & 13.6 & $\mathrm{~kg}$ \\
\hline FBE & Construction & milling, steel, average[RER] & 0.1 & $\mathrm{~kg}$ \\
\hline FBE & Construction & steel, converter, low-alloyed, at plant[RER] & 12.7 & $\mathrm{~kg}$ \\
\hline FBE & Construction & $\begin{array}{l}\text { aluminium, production mix, wrought alloy, at } \\
\text { plant[RER] }\end{array}$ & 5.02 & $\mathrm{~kg}$ \\
\hline FBE & Construction & synthetic rubber, at plant[RER] & 3 & $\mathrm{~kg}$ \\
\hline FBE & Construction & $\begin{array}{l}\text { heat, natural gas, at boiler fan burner low-NOx non- } \\
\text { modulating }<100 \mathrm{~kW}[\mathrm{RER}]\end{array}$ & $1.03 \mathrm{E}+03$ & MJ \\
\hline FBE & Construction & cast iron, at plant[RER] & 8.56 & $\mathrm{~kg}$ \\
\hline FBE & Construction & polyethylene, HDPE, granulate, at plant[RER] & 8.16 & $\mathrm{~kg}$ \\
\hline FBE & Construction & polyurethane, rigid foam, at plant[RER] & 4.04 & $\mathrm{~kg}$ \\
\hline FBE & Construction & steel, electric, un- and low-alloyed, at plant[RER] & 15.2 & $\mathrm{~kg}$ \\
\hline FBE & Construction & water, completely softened, at plant[RER] & 3.28 & $\mathrm{~kg}$ \\
\hline FBE & Construction & sheet rolling, steel[RER] & 9.07 & $\mathrm{~kg}$ \\
\hline FBE & Construction & copper, primary, at refinery[ID] & 1.34 & $\mathrm{~kg}$ \\
\hline FBE & Construction & alkyd paint, white, $60 \%$ in solvent, at plant[RER] & 2.9 & $\mathrm{~kg}$ \\
\hline FBE & Construction & cold impact extrusion, aluminium, 3 strokes[RER] & 4.58 & $\mathrm{~kg}$ \\
\hline FBE & Construction & steel, electric, un- and low-alloyed, at plant[RER] & 12.8 & $\mathrm{~kg}$ \\
\hline FBE & Construction & polypropylene, granulate, at plant[RER] & 6.12 & $\mathrm{~kg}$ \\
\hline FBE & Construction & milling, chromium steel, average[RER] & 0.05 & $\mathrm{~kg}$ \\
\hline FBE & Construction & glass fibre, at plant[RER] & 3.17 & $\mathrm{~kg}$ \\
\hline FBE & Construction & thermoforming, with calendering[RER] & 1.29 & $\mathrm{~kg}$ \\
\hline FBE & Construction & powder coating, steel[RER] & 1.2 & $\mathrm{~m} 2$ \\
\hline FBE & Construction & transport, freight, rail[CH] & 44.2 & tkm \\
\hline FBE & Construction & $\begin{array}{l}\text { acrylonitrile-butadiene-styrene copolymer, ABS, at } \\
\text { plant[RER] }\end{array}$ & 2.27 & $\mathrm{~kg}$ \\
\hline FBE & Construction & wire drawing, copper[RER] & 4.74 & $\mathrm{~kg}$ \\
\hline FBE & Construction & carbon black, at plant[GLO] & 5.21 & $\mathrm{~kg}$ \\
\hline FBE & Construction & aluminium, production mix, cast alloy, at plant[RER] & 2.02 & $\mathrm{~kg}$ \\
\hline FBE & Construction & water, completely softened, at plant[RER] & 1.57 & $\mathrm{~kg}$ \\
\hline FBE & Construction & $\begin{array}{l}\text { heat, natural gas, at boiler fan burner low-NOx non- } \\
\text { modulating }<100 \mathrm{~kW}[\mathrm{RER}]\end{array}$ & 391 & MJ \\
\hline FBE & Construction & transport, freight, rail $[\mathrm{CH}]$ & 34.3 & tkm \\
\hline FBE & Construction & $\begin{array}{l}\text { aluminium product manufacturing, average metal } \\
\text { working[RER] }\end{array}$ & 2.12 & $\mathrm{~kg}$ \\
\hline FBE & Construction & transport, freight, rail $[\mathrm{CH}]$ & 26.6 & $\mathrm{tkm}$ \\
\hline FBE & Construction & powder coating, steel[RER] & 0.69 & $\mathrm{~m} 2$ \\
\hline
\end{tabular}




\begin{tabular}{|c|c|c|c|c|}
\hline FBE & Construction & chromium steel 18/8, at plant[RER] & 2.85 & $\mathrm{~kg}$ \\
\hline FBE & Construction & transport, lorry 16-32t, EURO5[RER] & 19.6 & tkm \\
\hline FBE & Construction & $\begin{array}{l}\text { aluminium, production mix, wrought alloy, at } \\
\text { plant[RER] }\end{array}$ & 1.25 & $\mathrm{~kg}$ \\
\hline FBE & Construction & steel, converter, low-alloyed, at plant[RER] & 2.49 & $\mathrm{~kg}$ \\
\hline FBE & Construction & $\begin{array}{l}\text { steel product manufacturing, average metal } \\
\text { working[RER] }\end{array}$ & 2.48 & $\mathrm{~kg}$ \\
\hline FBE & Construction & chromium steel 18/8, at plant[RER] & 2.08 & $\mathrm{~kg}$ \\
\hline FBE & Construction & transport, freight, rail[CH] & 19.2 & tkm \\
\hline FBE & Construction & sheet rolling, steel[RER] & 2.28 & $\mathrm{~kg}$ \\
\hline FBE & Construction & $\begin{array}{l}\text { aluminium product manufacturing, average metal } \\
\text { working[RER] }\end{array}$ & 1.25 & $\mathrm{~kg}$ \\
\hline FBE & Construction & wire drawing, steel[RER] & 2.08 & $\mathrm{~kg}$ \\
\hline FBE & Construction & electricity, medium voltage, at grid[CH] & 18.5 & $\mathrm{kWh}$ \\
\hline FBE & Construction & nylon 6 , at plant [RER] & 0.4 & $\mathrm{~kg}$ \\
\hline FBE & Construction & neodymium oxide, at plant[CN] & 1.67 & $\mathrm{~kg}$ \\
\hline FBE & Construction & powder coating, steel[RER] & 0.357 & $\mathrm{~m} 2$ \\
\hline FBE & Construction & zinc oxide, at plant[RER] & 1 & $\mathrm{~kg}$ \\
\hline FBE & Construction & wire drawing, copper[RER] & 1.57 & $\mathrm{~kg}$ \\
\hline FBE & Construction & polystyrene, high impact, HIPS, at plant[RER] & 1.59 & $\mathrm{~kg}$ \\
\hline FBE & Construction & steel, electric, un- and low-alloyed, at plant[RER] & 2.49 & $\mathrm{~kg}$ \\
\hline FBE & Construction & gold, at regional storage[RER] & $6.00 \mathrm{E}-05$ & $\mathrm{~kg}$ \\
\hline FBE & Construction & transport, lorry 16-32t, EURO5[RER] & 8.84 & tkm \\
\hline FBE & Construction & tap water, at user[RER] & 0.47 & $\mathrm{~kg}$ \\
\hline FBE & Construction & copper, primary, at refinery[ID] & 0.2 & $\mathrm{~kg}$ \\
\hline FBE & Construction & copper, secondary, at refinery[RER] & 0.24 & $\mathrm{~kg}$ \\
\hline FBE & Construction & plywood, outdoor use, at plant[RER] & 0.002 & $\mathrm{~m} 3$ \\
\hline FBE & Construction & milling, chromium steel, average[RER] & 0.009 & $\mathrm{~kg}$ \\
\hline FBE & Construction & $\begin{array}{l}\text { heat, natural gas, at boiler fan burner low-NOx non- } \\
\text { modulating }<100 \mathrm{~kW}[\mathrm{RER}]\end{array}$ & 104 & MJ \\
\hline FBE & Construction & $\begin{array}{l}\text { acrylonitrile-butadiene-styrene copolymer, ABS, at } \\
\text { plant[RER] }\end{array}$ & 0.5 & $\mathrm{~kg}$ \\
\hline FBE & Construction & polymethyl methacrylate, beads, at plant[RER] & 0.39 & $\mathrm{~kg}$ \\
\hline FBE & Construction & transport, lorry 16-32t, EURO5[RER] & 6.87 & tkm \\
\hline FBE & Construction & tap water, at user[RER] & 0.37 & $\mathrm{~kg}$ \\
\hline FBE & Construction & copper, secondary, at refinery[RER] & 0.2 & $\mathrm{~kg}$ \\
\hline FBE & Construction & $\begin{array}{l}\text { copper product manufacturing, average metal } \\
\text { working[RER] }\end{array}$ & 0.2 & $\mathrm{~kg}$ \\
\hline FBE & Construction & extrusion, plastic pipes[RER] & 0.32 & $\mathrm{~kg}$ \\
\hline FBE & Construction & synthetic rubber, at plant[RER] & 0.23 & $\mathrm{~kg}$ \\
\hline FBE & Construction & silicon, electronic grade, at plant[DE] & 0.09 & $\mathrm{~kg}$ \\
\hline FBE & Construction & milling, aluminium, average[RER] & 0.14 & $\mathrm{~kg}$ \\
\hline FBE & Construction & cast iron, at plant[RER] & 0.62 & $\mathrm{~kg}$ \\
\hline FBE & Construction & magnesium-alloy, AZ91, diecasting, at plant[RER] & 0.15 & $\mathrm{~kg}$ \\
\hline FBE & Construction & nylon 66, at plant[RER] & 0.17 & $\mathrm{~kg}$ \\
\hline FBE & Construction & copper, primary, at refinery[ID] & 0.11 & $\mathrm{~kg}$ \\
\hline FBE & Construction & chromium steel 18/8, at plant[RER] & 0.62 & $\mathrm{~kg}$ \\
\hline
\end{tabular}




\begin{tabular}{|c|c|c|c|c|}
\hline FBE & Construction & $\begin{array}{l}\text { polyethylene terephthalate, granulate, amorphous, at } \\
\text { plant[RER] }\end{array}$ & 0.5 & $\mathrm{~kg}$ \\
\hline FBE & Construction & brass, at plant $[\mathrm{CH}]$ & 0.12 & $\mathrm{~kg}$ \\
\hline FBE & Construction & steel, converter, low-alloyed, at plant[RER] & 0.62 & $\mathrm{~kg}$ \\
\hline FBE & Construction & sheet rolling, aluminium[RER] & 0.34 & $\mathrm{~kg}$ \\
\hline FBE & Construction & paraffin, at plant[RER] & 0.94 & $\mathrm{~kg}$ \\
\hline FBE & Construction & transport, lorry 3.5-7.5t, EURO5[RER] & 3.83 & tkm \\
\hline FBE & Construction & chromium steel $18 / 8$, at plant[RER] & 0.45 & $\mathrm{~kg}$ \\
\hline FBE & Construction & zinc coating, coils[RER] & 0.13 & $\mathrm{~m} 2$ \\
\hline FBE & Construction & sinter, iron, at plant[GLO] & 6.25 & $\mathrm{~kg}$ \\
\hline FBE & Construction & lead, at regional storage[RER] & 0.19 & $\mathrm{~kg}$ \\
\hline FBE & Construction & steel, electric, un- and low-alloyed, at plant[RER] & 0.62 & $\mathrm{~kg}$ \\
\hline FBE & Construction & $\begin{array}{l}\text { heat, natural gas, at industrial furnace low-NOx } \\
>100 \mathrm{~kW}[\mathrm{RER}]\end{array}$ & 35.7 & MJ \\
\hline FBE & Construction & chemicals organic, at plant[GLO] & 0.08 & $\mathrm{~kg}$ \\
\hline FBE & Construction & naphtha, at refinery[RER] & 0.5 & $\mathrm{~kg}$ \\
\hline FBE & Construction & polyethylene, HDPE, granulate, at plant[RER] & 0.26 & $\mathrm{~kg}$ \\
\hline FBE & Construction & $\begin{array}{l}\text { metal product manufacturing, average metal } \\
\text { working[RER] }\end{array}$ & 0.26 & $\mathrm{~kg}$ \\
\hline FBE & Construction & glass fibre, at plant[RER] & 0.13 & $\mathrm{~kg}$ \\
\hline FBE & Construction & tap water, at user[RER] & 0.08 & $\mathrm{~kg}$ \\
\hline FBE & Construction & transport, freight, rail[CH] & 1.83 & tkm \\
\hline FBE & Construction & $\begin{array}{l}\text { polyethylene terephthalate, granulate, bottle grade, at } \\
\text { plant[RER] }\end{array}$ & 0.16 & $\mathrm{~kg}$ \\
\hline FBE & Construction & transport, lorry 16-32t, EURO5[RER] & 1.3 & tkm \\
\hline FBE & Construction & $\begin{array}{l}\text { heat, natural gas, at boiler fan burner low-NOx non- } \\
\text { modulating }<100 \mathrm{~kW}[\mathrm{RER}]\end{array}$ & 17.4 & MJ \\
\hline FBE & Construction & $\begin{array}{l}\text { heat, natural gas, at industrial furnace low-NOx } \\
>100 \mathrm{~kW}[\mathrm{RER}]\end{array}$ & 17 & MJ \\
\hline FBE & Construction & zinc, primary, at regional storage[RER] & 0.06 & $\mathrm{~kg}$ \\
\hline FBE & Construction & silicon, electronic grade, at plant[DE] & 0.016 & $\mathrm{~kg}$ \\
\hline FBE & Construction & tin, at regional storage[RER] & 0.007 & $\mathrm{~kg}$ \\
\hline FBE & Construction & milling, aluminium, average[RER] & 0.02 & $\mathrm{~kg}$ \\
\hline FBE & Construction & chemicals inorganic, at plant[GLO] & 0.08 & $\mathrm{~kg}$ \\
\hline FBE & Construction & copper, secondary, at refinery[RER] & 0.02 & $\mathrm{~kg}$ \\
\hline FBE & Construction & epoxy resin, liquid, at plant[RER] & 0.05 & $\mathrm{~kg}$ \\
\hline FBE & Construction & transport, lorry 16-32t, EURO5[RER] & 0.37 & tkm \\
\hline FBE & Construction & chromium steel 18/8, at plant[RER] & 0.05 & $\mathrm{~kg}$ \\
\hline FBE & Construction & bronze, at plant $[\mathrm{CH}]$ & 0.007 & $\mathrm{~kg}$ \\
\hline FBE & Construction & boric oxide, at plant[GLO] & 0.06 & $\mathrm{~kg}$ \\
\hline FBE & Construction & carbon dioxide liquid, at plant[RER] & 0.26 & $\mathrm{~kg}$ \\
\hline FBE & Construction & silver, at regional storage[RER] & $6.00 \mathrm{E}-05$ & $\mathrm{~kg}$ \\
\hline FBE & Construction & $\begin{array}{l}\text { natural gas, burned in industrial furnace low-NOx } \\
>100 \mathrm{~kW}[\mathrm{RER}]\end{array}$ & 1.8 & MJ \\
\hline FBE & Construction & zinc coating, pieces[RER] & 0.12 & $\mathrm{~m} 2$ \\
\hline FBE & Construction & secondary sulphur, at refinery[RER] & 0.23 & $\mathrm{~kg}$ \\
\hline FBE & Construction & printed wiring board, surface mounted, unspec., $\mathrm{Pb}$ & 0.4 & $\mathrm{~kg}$ \\
\hline
\end{tabular}




\begin{tabular}{|c|c|c|c|c|}
\hline & & free, at plant[GLO] & & \\
\hline FBE & Construction & degreasing, metal part in alkaline bath[RER] & 30.5 & $\mathrm{~m} 2$ \\
\hline FBE & Construction & nickel, $99.5 \%$, at plant[GLO] & 0.0006 & $\mathrm{~kg}$ \\
\hline FBE & Construction & $\begin{array}{l}\text { heat, natural gas, at industrial furnace low-NOx } \\
>100 \mathrm{~kW}[\mathrm{RER}]\end{array}$ & 1 & MJ \\
\hline FBE & Construction & carbon dioxide liquid, at plant[RER] & 0.12 & $\mathrm{~kg}$ \\
\hline FBE & Construction & lead, at regional storage[RER] & 0.004 & $\mathrm{~kg}$ \\
\hline FBE & Construction & nitrogen, liquid, at plant[RER] & 0.12 & $\mathrm{~kg}$ \\
\hline FBE & Construction & $\begin{array}{l}\text { compressed air, average generation, }>30 \mathrm{~kW}, 6 \text { bar } \\
\text { gauge, at compressor[RER] }\end{array}$ & 419 & $\mathrm{~m} 3$ \\
\hline FBE & Construction & resistor, unspecified, at plant[GLO] & 0.11 & $\mathrm{~kg}$ \\
\hline FBE & Construction & chromium, at regional storage[RER] & 0.0002 & $\mathrm{~kg}$ \\
\hline FBE & Construction & $\begin{array}{l}\text { natural gas, burned in industrial furnace low-NOx } \\
>100 \mathrm{~kW} \text { [RER] }\end{array}$ & 0.48 & MJ \\
\hline FBE & Construction & oxygen, liquid, at plant[RER] & 0.06 & $\mathrm{~kg}$ \\
\hline FBE & Construction & acetylene, at regional storehouse $[\mathrm{CH}]$ & 0.004 & $\mathrm{~kg}$ \\
\hline FBE & Construction & dolomite, at plant[RER] & 0.25 & $\mathrm{~kg}$ \\
\hline FBE & Construction & $\begin{array}{l}\text { compressed air, average generation, }<30 \mathrm{~kW}, 12 \text { bar } \\
\text { gauge, at compressor[RER] }\end{array}$ & 98.5 & $\mathrm{~m} 3$ \\
\hline FBE & Construction & degreasing, metal part in alkaline bath[RER] & 3.29 & $\mathrm{~m} 2$ \\
\hline FBE & Construction & $\begin{array}{l}\text { compressed air, average generation, }>30 \mathrm{~kW}, 6 \text { bar } \\
\text { gauge, at compressor[RER] }\end{array}$ & 69.6 & $\mathrm{~m} 3$ \\
\hline FBE & Construction & acetylene, at regional storehouse $[\mathrm{CH}]$ & 0.001 & $\mathrm{~kg}$ \\
\hline FBE & Construction & degreasing, metal part in alkaline bath[RER] & 1.34 & $\mathrm{~m} 2$ \\
\hline FBE & Construction & oxygen, liquid, at plant[RER] & 0.006 & $\mathrm{~kg}$ \\
\hline$\overline{\text { FBE }}$ & Construction & degreasing, metal part in alkaline bath[RER] & 0.69 & $\mathrm{~m} 2$ \\
\hline FBE & Construction & $\begin{array}{l}\text { compressed air, average generation, }<30 \mathrm{~kW}, 12 \text { bar } \\
\text { gauge, at compressor[RER] }\end{array}$ & 15.3 & $\mathrm{~m} 3$ \\
\hline FBE & Construction & zinc coating, pieces[RER] & 0.001 & $\mathrm{~m} 2$ \\
\hline FBE & Construction & $\begin{array}{l}\text { compressed air, average generation, }>30 \mathrm{~kW}, 6 \text { bar } \\
\text { gauge, at compressor[RER] }\end{array}$ & 8.49 & $\mathrm{~m} 3$ \\
\hline FBE & Construction & $\begin{array}{l}\text { compressed air, average generation, }<30 \mathrm{~kW}, 12 \text { bar } \\
\text { gauge, at compressor[RER] }\end{array}$ & 1.7 & $\mathrm{~m} 3$ \\
\hline FBE & Construction & acetylene, at regional storehouse $[\mathrm{CH}]$ & $1.50 \mathrm{E}-05$ & $\mathrm{~kg}$ \\
\hline FBE & Construction & oxygen, liquid, at plant[RER] & 0.0001 & $\mathrm{~kg}$ \\
\hline FBE & Construction & degreasing, metal part in alkaline bath[RER] & 0.01 & $\mathrm{~m} 2$ \\
\hline FBE & Construction & transport, lorry 16-32t, EURO5[RER] & $1.80 \mathrm{E}-05$ & tkm \\
\hline FBE & Construction & degreasing, metal part in alkaline bath[RER] & 0.006 & $\mathrm{~m} 2$ \\
\hline FBE & Construction & barite, at plant[RER] & $1.00 \mathrm{E}-08$ & $\mathrm{~kg}$ \\
\hline FBE & Use & electricity $\operatorname{mix}[\mathrm{UCTE}]$ & $2.60 \mathrm{E}+04$ & $\mathrm{kWh}$ \\
\hline FBE & Use & $\begin{array}{l}\text { electricity, low voltage, production UCTE, at } \\
\text { grid[UCTE] }\end{array}$ & 733 & $\mathrm{kWh}$ \\
\hline FBE & Use & tap water, at user[RER] & $1.50 \mathrm{E}+01$ & $\mathrm{~kg}$ \\
\hline FBE & EOL & $\begin{array}{l}\text { electricity, low voltage, production UCTE, at } \\
\text { grid[UCTE] }\end{array}$ & 324 & $\mathrm{kWh}$ \\
\hline FBE & EOL & $\begin{array}{l}\text { disposal, emulsion paint remains, } 0 \% \text { water, to } \\
\text { hazardous waste incineration[CH] }\end{array}$ & 100 & $\mathrm{~kg}$ \\
\hline FBE & EOL & $\begin{array}{l}\text { electricity, medium voltage, production UCTE, at } \\
\text { grid[UCTE] }\end{array}$ & 85.8 & $\mathrm{kWh}$ \\
\hline
\end{tabular}




\begin{tabular}{|c|c|c|c|c|}
\hline FBE & EOL & $\begin{array}{l}\text { disposal, plastics, mixture, } 15.3 \% \text { water, to municipal } \\
\text { incineration }[\mathrm{CH}]\end{array}$ & 65 & $\mathrm{~kg}$ \\
\hline FBE & EOL & transport, lorry 16-32t, EURO5[RER] & 32.6 & tkm \\
\hline FBE & EOL & $\begin{array}{l}\text { disposal, glass, } 0 \% \text { water, to municipal } \\
\text { incineration }[\mathrm{CH}]\end{array}$ & 30.1 & $\mathrm{~kg}$ \\
\hline FBE & EOL & $\begin{array}{l}\text { disposal, rubber, unspecified, } 0 \% \text { water, to municipal } \\
\text { incineration }[\mathrm{CH}]\end{array}$ & 30 & $\mathrm{~kg}$ \\
\hline FBE & EOL & $\begin{array}{l}\text { disposal, zinc in car shredder residue, } 0 \% \text { water, to } \\
\text { municipal incineration }[\mathrm{CH}]\end{array}$ & 5.89 & $\mathrm{~kg}$ \\
\hline FBE & EOL & $\begin{array}{l}\text { disposal, wire plastic, } 3.55 \% \text { water, to municipal } \\
\text { incineration }[\mathrm{CH}]\end{array}$ & 2 & $\mathrm{~kg}$ \\
\hline FBE & EOL & $\begin{array}{l}\text { disposal, antifreezer liquid, } 51.8 \% \text { water, to } \\
\text { hazardous waste incineration }[\mathrm{CH}]\end{array}$ & 1 & $\mathrm{~kg}$ \\
\hline FBE & EOL & transport, lorry $3.5-20 \mathrm{t}$, fleet average $[\mathrm{CH}]$ & 0.7 & $\mathrm{tkm}$ \\
\hline FBE & EOL & nitrogen, liquid, at plant[RER] & 1.12 & $\mathrm{~kg}$ \\
\hline FBE & EOL & chemicals inorganic, at plant[GLO] & 0.025 & $\mathrm{~kg}$ \\
\hline FBE & EOL & sulphuric acid, liquid, at plant[RER] & 0.23 & $\mathrm{~kg}$ \\
\hline FBE & EOL & $\begin{array}{l}\text { electricity, medium voltage, production UCTE, at } \\
\text { grid[UCTE] }\end{array}$ & 0.14 & $\mathrm{kWh}$ \\
\hline FBE & EOL & lime, hydrated, packed, at plant $[\mathrm{CH}]$ & 0.116 & $\mathrm{~kg}$ \\
\hline FBE & EOL & electricity, low voltage, at grid[CH] & 0.07 & $\mathrm{kWh}$ \\
\hline FBE & EOL & shredding, electrical and electronic scrap[GLO] & 1 & $\mathrm{~kg}$ \\
\hline FBE & EOL & disposal, electronics for control units[RER] & 5 & $\mathrm{~kg}$ \\
\hline FBE & EOL & $\begin{array}{l}\text { disposal, catalytic converter for cars, } 0 \% \text { water, to } \\
\text { underground deposit[DE] }\end{array}$ & 3 & $\mathrm{~kg}$ \\
\hline HFC & Construction & milling, steel, average[RER] & 56.5 & $\mathrm{~kg}$ \\
\hline HFC & Construction & injection moulding[RER] & 43.5 & $\mathrm{~kg}$ \\
\hline HFC & Construction & milling, steel, average[RER] & 1.27 & $\mathrm{~kg}$ \\
\hline HFC & Construction & ferrite, at plant[GLO] & 35.6 & $\mathrm{~kg}$ \\
\hline HFC & Construction & synthetic rubber, at plant[RER] & 30.5 & $\mathrm{~kg}$ \\
\hline HFC & Construction & graphite, at plant[RER] & 14 & $\mathrm{~kg}$ \\
\hline HFC & Construction & steel, converter, low-alloyed, at plant[RER] & 74.9 & $\mathrm{~kg}$ \\
\hline HFC & Construction & injection moulding[RER] & 12.6 & $\mathrm{~kg}$ \\
\hline HFC & Construction & $\begin{array}{l}\text { steel product manufacturing, average metal } \\
\text { working[RER] }\end{array}$ & 67.8 & $\mathrm{~kg}$ \\
\hline HFC & Construction & milling, steel, average[RER] & 0.51 & $\mathrm{~kg}$ \\
\hline HFC & Construction & $\begin{array}{l}\text { steel product manufacturing, average metal } \\
\text { working[RER] }\end{array}$ & 63.4 & $\mathrm{~kg}$ \\
\hline HFC & Construction & tempering, flat glass[RER] & 15.2 & $\mathrm{~kg}$ \\
\hline HFC & Construction & flat glass, uncoated, at plant[RER] & 15.2 & $\mathrm{~kg}$ \\
\hline HFC & Construction & electricity, medium voltage, at grid[CH] & 443 & $\mathrm{kWh}$ \\
\hline HFC & Construction & water, completely softened, at plant[RER] & 14.7 & $\mathrm{~kg}$ \\
\hline HFC & Construction & copper, at regional storage[RER] & 7.6 & $\mathrm{~kg}$ \\
\hline HFC & Construction & glass fibre, at plant[RER] & 18 & $\mathrm{~kg}$ \\
\hline HFC & Construction & $\begin{array}{l}\text { polyethylene terephthalate, granulate, bottle grade, at } \\
\text { plant[RER] }\end{array}$ & 25 & $\mathrm{~kg}$ \\
\hline HFC & Construction & steel, electric, un- and low-alloyed, at plant[RER] & 51 & $\mathrm{~kg}$ \\
\hline HFC & Construction & steel, converter, low-alloyed, at plant[RER] & 31.7 & $\mathrm{~kg}$ \\
\hline
\end{tabular}




\begin{tabular}{|c|c|c|c|c|}
\hline HFC & Construction & sheet rolling, steel[RER] & 28.2 & $\mathrm{~kg}$ \\
\hline HFC & Construction & zinc coating, pieces[RER] & 192 & $\mathrm{~m} 2$ \\
\hline HFC & Construction & copper, primary, at refinery[ID] & 4.03 & $\mathrm{~kg}$ \\
\hline HFC & Construction & chromium steel 18/8, at plant[RER] & 22 & $\mathrm{~kg}$ \\
\hline HFC & Construction & steel, low-alloyed, at plant[RER] & 35 & $\mathrm{~kg}$ \\
\hline HFC & Construction & ethylene glycol, at plant[RER] & 37 & $\mathrm{~kg}$ \\
\hline $\mathrm{HFC}$ & Construction & $\begin{array}{l}\text { steel product manufacturing, average metal } \\
\text { working[RER] }\end{array}$ & 23.6 & $\mathrm{~kg}$ \\
\hline $\mathrm{HFC}$ & Construction & alkyd paint, white, $60 \%$ in solvent, at plant[RER] & 7.86 & $\mathrm{~kg}$ \\
\hline HFC & Construction & electricity mix[UCTE] & 160 & $\mathrm{kWh}$ \\
\hline HFC & Construction & milling, cast iron, average[RER] & 0.17 & $\mathrm{~kg}$ \\
\hline HFC & Construction & synthetic rubber, at plant[RER] & 5.66 & $\mathrm{~kg}$ \\
\hline HFC & Construction & hot rolling, steel[RER] & 20.2 & $\mathrm{~kg}$ \\
\hline HFC & Construction & steel, electric, un- and low-alloyed, at plant[RER] & 31.7 & $\mathrm{~kg}$ \\
\hline HFC & Construction & aluminium alloy, $\mathrm{AlMg} 3$, at plant[RER] & 10 & $\mathrm{~kg}$ \\
\hline HFC & Construction & platinum, secondary, at refinery[RER] & 0.006 & $\mathrm{~kg}$ \\
\hline HFC & Construction & steel, converter, low-alloyed, at plant[RER] & 15.9 & $\mathrm{~kg}$ \\
\hline HFC & Construction & $\begin{array}{l}\text { steel product manufacturing, average metal } \\
\text { working[RER] }\end{array}$ & 15.9 & $\mathrm{~kg}$ \\
\hline HFC & Construction & powder coating, steel[RER] & 3.23 & $\mathrm{~m} 2$ \\
\hline HFC & Construction & injection moulding[RER] & 2.5 & $\mathrm{~kg}$ \\
\hline HFC & Construction & electricity, medium voltage, at grid[UCTE] & 146 & $\mathrm{kWh}$ \\
\hline HFC & Construction & $\begin{array}{l}\text { steel product manufacturing, average metal } \\
\text { working[RER] }\end{array}$ & 13.6 & $\mathrm{~kg}$ \\
\hline HFC & Construction & milling, steel, average[RER] & 0.1 & $\mathrm{~kg}$ \\
\hline HFC & Construction & steel, converter, low-alloyed, at plant[RER] & 12.7 & $\mathrm{~kg}$ \\
\hline HFC & Construction & tetrafluoroethylene, at plant[RER] & 8 & $\mathrm{~kg}$ \\
\hline $\mathrm{HFC}$ & Construction & $\begin{array}{l}\text { aluminium, production mix, wrought alloy, at } \\
\text { plant[RER] }\end{array}$ & 5.02 & $\mathrm{~kg}$ \\
\hline HFC & Construction & synthetic rubber, at plant[RER] & 3 & $\mathrm{~kg}$ \\
\hline HFC & Construction & $\begin{array}{l}\text { heat, natural gas, at boiler fan burner low-NOx non- } \\
\text { modulating }<100 \mathrm{~kW}[\mathrm{RER}]\end{array}$ & $1.03 \mathrm{E}+03$ & MJ \\
\hline HFC & Construction & cast iron, at plant[RER] & 8.56 & $\mathrm{~kg}$ \\
\hline HFC & Construction & polyurethane, rigid foam, at plant[RER] & 4.04 & $\mathrm{~kg}$ \\
\hline HFC & Construction & water, completely softened, at plant[RER] & 3.28 & $\mathrm{~kg}$ \\
\hline HFC & Construction & sheet rolling, steel[RER] & 9.07 & $\mathrm{~kg}$ \\
\hline HFC & Construction & copper, primary, at refinery[ID] & 1.34 & $\mathrm{~kg}$ \\
\hline HFC & Construction & cold impact extrusion, aluminium, 3 strokes[RER] & 4.58 & $\mathrm{~kg}$ \\
\hline HFC & Construction & steel, electric, un- and low-alloyed, at plant[RER] & 12.8 & $\mathrm{~kg}$ \\
\hline HFC & Construction & polypropylene, granulate, at plant[RER] & 6.12 & $\mathrm{~kg}$ \\
\hline HFC & Construction & milling, chromium steel, average[RER] & 0.05 & $\mathrm{~kg}$ \\
\hline $\mathrm{HFC}$ & Construction & thermoforming, with calendering[RER] & 1.29 & $\mathrm{~kg}$ \\
\hline HFC & Construction & tube insulation, elastomere, at plant[DE] & 0.5 & $\mathrm{~kg}$ \\
\hline HFC & Construction & powder coating, steel[RER] & 1.2 & $\mathrm{~m} 2$ \\
\hline $\mathrm{HFC}$ & Construction & transport, freight, rail[CH] & 44.2 & tkm \\
\hline $\mathrm{HFC}$ & Construction & $\begin{array}{l}\text { acrylonitrile-butadiene-styrene copolymer, ABS, at } \\
\text { plant[RER] }\end{array}$ & 2.27 & $\mathrm{~kg}$ \\
\hline
\end{tabular}




\begin{tabular}{|c|c|c|c|c|}
\hline HFC & Construction & wire drawing, copper[RER] & 4.74 & $\mathrm{~kg}$ \\
\hline HFC & Construction & carbon black, at plant[GLO] & 5.21 & $\mathrm{~kg}$ \\
\hline HFC & Construction & aluminium, production mix, cast alloy, at plant[RER] & 2.02 & $\mathrm{~kg}$ \\
\hline HFC & Construction & water, completely softened, at plant[RER] & 1.57 & $\mathrm{~kg}$ \\
\hline HFC & Construction & $\begin{array}{l}\text { heat, natural gas, at boiler fan burner low-NOx non- } \\
\text { modulating }<100 \mathrm{~kW}[\mathrm{RER}]\end{array}$ & 391 & MJ \\
\hline HFC & Construction & transport, freight, rail $[\mathrm{CH}]$ & 34.3 & tkm \\
\hline HFC & Construction & $\begin{array}{l}\text { aluminium product manufacturing, average metal } \\
\text { working[RER] }\end{array}$ & 2.12 & $\mathrm{~kg}$ \\
\hline $\mathrm{HFC}$ & Construction & transport, freight, rail[CH] & 26.6 & tkm \\
\hline HFC & Construction & powder coating, steel[RER] & 0.69 & $\mathrm{~m} 2$ \\
\hline $\mathrm{HFC}$ & Construction & transport, lorry 16-32t, EURO5[RER] & 19.6 & tkm \\
\hline HFC & Construction & $\begin{array}{l}\text { aluminium, production mix, wrought alloy, at } \\
\text { plant[RER] }\end{array}$ & 1.25 & $\mathrm{~kg}$ \\
\hline HFC & Construction & steel, converter, low-alloyed, at plant[RER] & 2.49 & $\mathrm{~kg}$ \\
\hline HFC & Construction & chromium steel 18/8, at plant[RER] & 2.08 & $\mathrm{~kg}$ \\
\hline HFC & Construction & transport, freight, rail $[\mathrm{CH}]$ & 19.2 & tkm \\
\hline HFC & Construction & sheet rolling, steel[RER] & 2.28 & $\mathrm{~kg}$ \\
\hline HFC & Construction & $\begin{array}{l}\text { aluminium product manufacturing, average metal } \\
\text { working[RER] }\end{array}$ & 1.25 & $\mathrm{~kg}$ \\
\hline HFC & Construction & wire drawing, steel[RER] & 2.08 & $\mathrm{~kg}$ \\
\hline HFC & Construction & nylon 6 , at plant[RER] & 0.4 & $\mathrm{~kg}$ \\
\hline HFC & Construction & powder coating, steel[RER] & 0.357 & $\mathrm{~m} 2$ \\
\hline HFC & Construction & zinc oxide, at plant[RER] & 1 & $\mathrm{~kg}$ \\
\hline HFC & Construction & wire drawing, copper[RER] & 1.57 & $\mathrm{~kg}$ \\
\hline HFC & Construction & polystyrene, high impact, HIPS, at plant[RER] & 1.59 & $\mathrm{~kg}$ \\
\hline HFC & Construction & steel, electric, un- and low-alloyed, at plant[RER] & 2.49 & $\mathrm{~kg}$ \\
\hline HFC & Construction & transport, lorry 16-32t, EURO5[RER] & 8.84 & tkm \\
\hline HFC & Construction & tap water, at user[RER] & 0.47 & $\mathrm{~kg}$ \\
\hline HFC & Construction & copper, primary, at refinery[ID] & 0.2 & $\mathrm{~kg}$ \\
\hline HFC & Construction & copper, secondary, at refinery[RER] & 0.24 & $\mathrm{~kg}$ \\
\hline HFC & Construction & reinforcing steel, at plant[RER] & 1 & $\mathrm{~kg}$ \\
\hline HFC & Construction & milling, chromium steel, average[RER] & 0.009 & $\mathrm{~kg}$ \\
\hline HFC & Construction & $\begin{array}{l}\text { heat, natural gas, at boiler fan burner low-NOx non- } \\
\text { modulating }<100 \mathrm{~kW}[\mathrm{RER}]\end{array}$ & 104 & MJ \\
\hline HFC & Construction & $\begin{array}{l}\text { acrylonitrile-butadiene-styrene copolymer, } \mathrm{ABS} \text {, at } \\
\text { plant[RER] }\end{array}$ & 0.5 & $\mathrm{~kg}$ \\
\hline HFC & Construction & polymethyl methacrylate, beads, at plant[RER] & 0.39 & $\mathrm{~kg}$ \\
\hline HFC & Construction & transport, lorry 16-32t, EURO5[RER] & 6.87 & tkm \\
\hline $\mathrm{HFC}$ & Construction & tap water, at user[RER] & 0.37 & $\mathrm{~kg}$ \\
\hline HFC & Construction & copper, secondary, at refinery[RER] & 0.2 & $\mathrm{~kg}$ \\
\hline HFC & Construction & $\begin{array}{l}\text { copper product manufacturing, average metal } \\
\text { working[RER] }\end{array}$ & 0.2 & $\mathrm{~kg}$ \\
\hline HFC & Construction & extrusion, plastic pipes[RER] & 0.32 & $\mathrm{~kg}$ \\
\hline HFC & Construction & synthetic rubber, at plant[RER] & 0.23 & $\mathrm{~kg}$ \\
\hline HFC & Construction & silicon, electronic grade, at plant[DE] & 0.09 & $\mathrm{~kg}$ \\
\hline HFC & Construction & milling, aluminium, average[RER] & 0.14 & $\mathrm{~kg}$ \\
\hline
\end{tabular}




\begin{tabular}{|c|c|c|c|c|}
\hline HFC & Construction & magnesium-alloy, AZ91, diecasting, at plant[RER] & 0.15 & $\mathrm{~kg}$ \\
\hline HFC & Construction & nylon 66, at plant[RER] & 0.17 & $\mathrm{~kg}$ \\
\hline HFC & Construction & copper, primary, at refinery[ID] & 0.11 & $\mathrm{~kg}$ \\
\hline HFC & Construction & silica sand, at plant[DE] & 0.5 & $\mathrm{~kg}$ \\
\hline HFC & Construction & $\begin{array}{l}\text { polyethylene terephthalate, granulate, amorphous, at } \\
\text { plant[RER] }\end{array}$ & 0.5 & $\mathrm{~kg}$ \\
\hline HFC & Construction & sheet rolling, aluminium[RER] & 0.34 & $\mathrm{~kg}$ \\
\hline HFC & Construction & paraffin, at plant[RER] & 0.94 & $\mathrm{~kg}$ \\
\hline HFC & Construction & transport, lorry 3.5-7.5t, EURO5[RER] & 3.83 & $\mathrm{tkm}$ \\
\hline HFC & Construction & chromium steel 18/8, at plant[RER] & 0.45 & $\mathrm{~kg}$ \\
\hline HFC & Construction & zinc coating, coils[RER] & 0.13 & $\mathrm{~m} 2$ \\
\hline HFC & Construction & lead, at regional storage[RER] & 0.19 & $\mathrm{~kg}$ \\
\hline $\mathrm{HFC}$ & Construction & $\begin{array}{l}\text { heat, natural gas, at industrial furnace low-NOx } \\
>100 \mathrm{~kW}[\mathrm{RER}]\end{array}$ & 35.7 & MJ \\
\hline HFC & Construction & naphtha, at refinery[RER] & 0.5 & $\mathrm{~kg}$ \\
\hline HFC & Construction & polyethylene, HDPE, granulate, at plant[RER] & 0.26 & $\mathrm{~kg}$ \\
\hline HFC & Construction & glass fibre, at plant[RER] & 0.13 & $\mathrm{~kg}$ \\
\hline $\mathrm{HFC}$ & Construction & tap water, at user[RER] & 0.08 & $\mathrm{~kg}$ \\
\hline HFC & Construction & $\begin{array}{l}\text { polyethylene terephthalate, granulate, bottle grade, at } \\
\text { plant[RER] }\end{array}$ & 0.16 & $\mathrm{~kg}$ \\
\hline HFC & Construction & transport, lorry 16-32t, EURO5[RER] & 1.3 & tkm \\
\hline HFC & Construction & $\begin{array}{l}\text { heat, natural gas, at industrial furnace low-NOx } \\
>100 \mathrm{~kW}[\mathrm{RER}]\end{array}$ & 17 & $\mathrm{MJ}$ \\
\hline HFC & Construction & zinc, primary, at regional storage[RER] & 0.06 & $\mathrm{~kg}$ \\
\hline $\mathrm{HFC}$ & Construction & milling, aluminium, average[RER] & 0.02 & $\mathrm{~kg}$ \\
\hline HFC & Construction & copper, secondary, at refinery[RER] & 0.02 & $\mathrm{~kg}$ \\
\hline HFC & Construction & chromium steel $18 / 8$, at plant[RER] & 0.05 & $\mathrm{~kg}$ \\
\hline HFC & Construction & electronics for control units[RER] & 4 & $\mathrm{~kg}$ \\
\hline HFC & Construction & carbon dioxide liquid, at plant[RER] & 0.26 & $\mathrm{~kg}$ \\
\hline HFC & Construction & $\begin{array}{l}\text { natural gas, burned in industrial furnace low-NOx } \\
>100 \mathrm{~kW}[\mathrm{RER}]\end{array}$ & 1.8 & $\mathrm{MJ}$ \\
\hline HFC & Construction & zinc coating, pieces[RER] & 0.12 & $\mathrm{~m} 2$ \\
\hline HFC & Construction & secondary sulphur, at refinery[RER] & 0.23 & $\mathrm{~kg}$ \\
\hline HFC & Construction & degreasing, metal part in alkaline bath[RER] & 30.5 & $\mathrm{~m} 2$ \\
\hline HFC & Construction & $\begin{array}{l}\text { heat, natural gas, at industrial furnace low-NOx } \\
>100 \mathrm{~kW}[\mathrm{RER}]\end{array}$ & 1 & $\mathrm{MJ}$ \\
\hline HFC & Construction & carbon dioxide liquid, at plant[RER] & 0.12 & $\mathrm{~kg}$ \\
\hline HFC & Construction & nitrogen, liquid, at plant[RER] & 0.12 & $\mathrm{~kg}$ \\
\hline HFC & Construction & $\begin{array}{l}\text { compressed air, average generation, }>30 \mathrm{~kW}, 6 \text { bar } \\
\text { gauge, at compressor[RER] }\end{array}$ & 419 & $\mathrm{~m} 3$ \\
\hline HFC & Construction & $\begin{array}{l}\text { natural gas, burned in industrial furnace low-NOx } \\
>100 \mathrm{~kW}[\mathrm{RER}]\end{array}$ & 0.48 & $\mathrm{MJ}$ \\
\hline HFC & Construction & oxygen, liquid, at plant[RER] & 0.06 & $\mathrm{~kg}$ \\
\hline HFC & Construction & acetylene, at regional storehouse $[\mathrm{CH}]$ & 0.004 & $\mathrm{~kg}$ \\
\hline HFC & Construction & dolomite, at plant[RER] & 0.25 & $\mathrm{~kg}$ \\
\hline HFC & Construction & $\begin{array}{l}\text { compressed air, average generation, }<30 \mathrm{~kW}, 12 \text { bar } \\
\text { gauge, at compressor[RER] }\end{array}$ & 98.5 & $\mathrm{~m} 3$ \\
\hline $\mathrm{HFC}$ & Construction & degreasing, metal part in alkaline bath[RER] & 3.29 & $\mathrm{~m} 2$ \\
\hline
\end{tabular}




\begin{tabular}{|c|c|c|c|c|}
\hline HFC & Construction & $\begin{array}{l}\text { compressed air, average generation, }>30 \mathrm{~kW}, 6 \text { bar } \\
\text { gauge, at compressor[RER] }\end{array}$ & 69.6 & $\mathrm{~m} 3$ \\
\hline HFC & Construction & acetylene, at regional storehouse $[\mathrm{CH}]$ & 0.001 & $\mathrm{~kg}$ \\
\hline HFC & Construction & degreasing, metal part in alkaline bath[RER] & 1.34 & $\mathrm{~m} 2$ \\
\hline HFC & Construction & oxygen, liquid, at plant[RER] & 0.006 & $\mathrm{~kg}$ \\
\hline HFC & Construction & degreasing, metal part in alkaline bath[RER] & 0.69 & $\mathrm{~m} 2$ \\
\hline HFC & Construction & $\begin{array}{l}\text { compressed air, average generation, }<30 \mathrm{~kW}, 12 \text { bar } \\
\text { gauge, at compressor[RER] }\end{array}$ & 15.3 & $\mathrm{~m} 3$ \\
\hline HFC & Construction & $\begin{array}{l}\text { compressed air, average generation, }>30 \mathrm{~kW}, 6 \text { bar } \\
\text { gauge, at compressor[RER] }\end{array}$ & 8.49 & $\mathrm{~m} 3$ \\
\hline HFC & Construction & $\begin{array}{l}\text { compressed air, average generation, }<30 \mathrm{~kW}, 12 \text { bar } \\
\text { gauge, at compressor[RER] }\end{array}$ & 1.7 & $\mathrm{~m} 3$ \\
\hline HFC & Construction & acetylene, at regional storehouse $[\mathrm{CH}]$ & $1.50 \mathrm{E}-05$ & $\mathrm{~kg}$ \\
\hline HFC & Construction & oxygen, liquid, at plant[RER] & 0.0001 & $\mathrm{~kg}$ \\
\hline HFC & Construction & degreasing, metal part in alkaline bath[RER] & 0.01 & $\mathrm{~m} 2$ \\
\hline HFC & Construction & transport, lorry 16-32t, EURO5[RER] & $1.80 \mathrm{E}-05$ & tkm \\
\hline HFC & Construction & barite, at plant[RER] & $1.00 \mathrm{E}-08$ & $\mathrm{~kg}$ \\
\hline HFC & EOL & $\begin{array}{l}\text { electricity, low voltage, production UCTE, at } \\
\text { grid[UCTE] }\end{array}$ & 324 & $\mathrm{kWh}$ \\
\hline HFC & EOL & $\begin{array}{l}\text { disposal, emulsion paint remains, } 0 \% \text { water, to } \\
\text { hazardous waste incineration }[\mathrm{CH}]\end{array}$ & 100 & $\mathrm{~kg}$ \\
\hline HFC & EOL & $\begin{array}{l}\text { electricity, medium voltage, production UCTE, at } \\
\text { grid[UCTE] }\end{array}$ & 85.8 & $\mathrm{kWh}$ \\
\hline HFC & EOL & $\begin{array}{l}\text { disposal, plastics, mixture, } 15.3 \% \text { water, to municipal } \\
\text { incineration[CH] }\end{array}$ & 65 & $\mathrm{~kg}$ \\
\hline HFC & EOL & transport, lorry 16-32t, EURO5[RER] & 32.6 & tkm \\
\hline HFC & EOL & $\begin{array}{l}\text { disposal, glass, } 0 \% \text { water, to municipal } \\
\text { incineration }[\mathrm{CH}]\end{array}$ & 30.1 & $\mathrm{~kg}$ \\
\hline HFC & EOL & $\begin{array}{l}\text { disposal, rubber, unspecified, } 0 \% \text { water, to municipal } \\
\text { incineration }[\mathrm{CH}]\end{array}$ & 30 & $\mathrm{~kg}$ \\
\hline HFC & EOL & $\begin{array}{l}\text { disposal, zinc in car shredder residue, } 0 \% \text { water, to } \\
\text { municipal incineration }[\mathrm{CH}]\end{array}$ & 5.89 & $\mathrm{~kg}$ \\
\hline HFC & EOL & $\begin{array}{l}\text { disposal, wire plastic, } 3.55 \% \text { water, to municipal } \\
\text { incineration }[\mathrm{CH}]\end{array}$ & 2 & $\mathrm{~kg}$ \\
\hline HFC & EOL & $\begin{array}{l}\text { disposal, antifreezer liquid, } 51.8 \% \text { water, to } \\
\text { hazardous waste incineration }[\mathrm{CH}]\end{array}$ & 1 & $\mathrm{~kg}$ \\
\hline HFC & EOL & transport, lorry 3.5-20t, fleet average $[\mathrm{CH}]$ & 0.7 & tkm \\
\hline HFC & EOL & nitrogen, liquid, at plant[RER] & 1.12 & $\mathrm{~kg}$ \\
\hline HFC & EOL & chemicals inorganic, at plant[GLO] & 0.025 & $\mathrm{~kg}$ \\
\hline HFC & EOL & sulphuric acid, liquid, at plant[RER] & 0.23 & $\mathrm{~kg}$ \\
\hline HFC & EOL & $\begin{array}{l}\text { electricity, medium voltage, production UCTE, at } \\
\text { grid[UCTE] }\end{array}$ & 0.14 & $\mathrm{kWh}$ \\
\hline HFC & EOL & lime, hydrated, packed, at plant $[\mathrm{CH}]$ & 0.116 & $\mathrm{~kg}$ \\
\hline HFC & EOL & electricity, low voltage, at grid[UCTE] & 0.07 & $\mathrm{kWh}$ \\
\hline HFC & EOL & shredding, electrical and electronic scrap[GLO] & 1 & $\mathrm{~kg}$ \\
\hline HFC & EOL & disposal, electronics for control units[RER] & 5 & $\mathrm{~kg}$ \\
\hline HFC & EOL & $\begin{array}{l}\text { disposal, catalytic converter for cars, } 0 \% \text { water, to } \\
\text { underground deposit[DE] }\end{array}$ & 3 & $\mathrm{~kg}$ \\
\hline
\end{tabular}


Supporting information S2: Total cost of ownership calculations

Table S2.1. Disaggregated total cost of ownership (TCO) for the year 2020 and segment C/D, excluding all taxes (in thousand euros)

\begin{tabular}{|c|c|c|c|c|c|c|}
\hline Technology & Purchase $^{a}$ & Maintenance $^{\mathrm{a}}$ & Fuel $^{\mathrm{a}}$ & Infrastructure ${ }^{a}$ & Subsidy $^{b}$ & TCO \\
\hline FBE & 28.9 & 3.7 & 3.4 & 2.5 & 3.2 & 35.3 \\
\hline HFC & 30.9 & 4.5 & 5.6 & 2.7 & 3.2 & 40.5 \\
\hline ICE - gasoline & 21.4 & 5.5 & 4.7 & 0.6 & 0 & 32.2 \\
\hline ICE - diesel & 21.9 & 5.7 & 4.7 & 0.5 & 0 & 32.8 \\
\hline$\overline{I C E}-\operatorname{mix}^{\mathrm{c}}$ & 21.7 & 5.6 & 4.7 & 0.5 & 0 & 32.5 \\
\hline
\end{tabular}

${ }^{\mathrm{a}}$ Obtained from McKensey and Company ${ }^{3}$.

${ }^{\mathrm{b}}$ See Table S2.2.

${ }^{\mathrm{c}}$ Considering market diffusion for the year 2020 from European Commission ${ }^{4}$.

Table S2.2. National purchasing subsidies (electric car compared to internal combustion engine car) for electric vehicles in the EU-27 member states (as of January 2014)

\begin{tabular}{|l|c|c|c|}
\hline Member state & $\begin{array}{l}\text { One-time benefit } \\
\left(\boldsymbol{\epsilon} / \text { vehicle }^{\mathbf{a}}\right.\end{array}$ & $\begin{array}{l}\text { Recurring benefit } \\
\left(\boldsymbol{\epsilon} / \text { vehicle }^{\mathbf{a}}\right.\end{array}$ & Total (€/vehicle) \\
\hline Denmark & 15,260 & 390 & 15,650 \\
\hline France & 6,500 & 0 & 6,500 \\
\hline Great Britain & 6,022 & 0 & 6,022 \\
\hline Netherlands & 2,625 & 2,740 & 5,365 \\
\hline Italy & 3,000 & 810 & 3,810 \\
\hline Portugal & 519 & 495 & 1,014 \\
\hline Germany & 0 & 150 & 150 \\
\hline EU-27 $^{\mathbf{b}}$ & 3,105 & 128 & 3,233 \\
\hline
\end{tabular}

${ }^{\mathrm{a}}$ Obtained from ARF/M\&C ${ }^{5}$.

${ }^{\mathrm{b}}$ Average weighted value according to electric car diffusion in each member state for the year 2020 . Market diffusion values obtained from European Commission ${ }^{4}$. 
Supporting information S3: Model description, background data and results for the AIDS model

In a budget share $(M B S)$ form, the AIDS model for the $i$ th consumption group and a time period $t$ can be expressed as

$$
M B S_{t}^{i}=\alpha^{i}+\sum_{S=1, \ldots, n} \gamma_{s}^{i} \ln p_{t}^{S}+\beta^{i} \ln \left(\frac{x_{t}^{S}}{P_{t}}\right) \quad(S 3-1)
$$

where $n$ equals the total number of consumption groups, $x$ denotes total expenditures, $P$ is defined here as the Stone's price index, $p$ represents the price of a given group and $\alpha, \beta$ and $\gamma$ are the unknown parameters. The Stone's price index is defined as

$$
\ln P_{t}=\sum_{s=1, . ., n} M B S_{t}^{S} \ln p_{t}^{S} \quad(S 3-2)
$$

To comply with consumer demand theory, three restrictions are imposed: adding-up, homogeneity and symmetry:

$$
\begin{gathered}
\text { Adding up: } \sum_{S} w_{S}=1 ; \sum_{S} \alpha_{s}=1 ; \sum_{S} \beta_{s}=0 ; \sum_{S} \gamma_{s}^{i}=1 \quad(S 3-4) \\
\text { Homogeneity: } \sum_{i} \gamma_{s}^{i}=1 \\
\text { Symmetry: } \gamma_{s}^{i}=\gamma_{i}^{S}
\end{gathered}
$$

The adding up restriction imposes that the sum of the expenditures for each group equals to total expenditure. The homogeneity restriction imposes that demand levels remain unchanged if both prices and total expenditure change proportionally. The symmetry restriction imposes that the compensated cross-price elasticities between two consumption groups are equal. 
Table S3.1. Final consumption total expenditure and shares for the $1995-2012$ period and for the EU-27 by COICOP 2 digit classification. Source: Eurostat ${ }^{6}$

\begin{tabular}{|c|c|c|c|c|c|c|c|c|c|c|c|c|c|}
\hline \multirow[t]{2}{*}{ Year } & \multirow{2}{*}{$\begin{array}{c}\text { Total } \\
\text { expenditure } \\
(€)\end{array}$} & \multicolumn{12}{|c|}{ Shares } \\
\hline & & $\begin{array}{l}\text { CP01 Food } \\
\text { and non- } \\
\text { alcoholic } \\
\text { beverages }\end{array}$ & $\begin{array}{c}\mathrm{CP02} \\
\text { Alcoholic } \\
\text { beverages, } \\
\text { tobacco } \\
\text { and } \\
\text { narcotics }\end{array}$ & $\begin{array}{c}\text { CP03 } \\
\text { Clothing } \\
\text { and } \\
\text { footwear }\end{array}$ & $\begin{array}{c}\text { CP04 } \\
\text { Housing, } \\
\text { water, } \\
\text { electricity, } \\
\text { gas and } \\
\text { other fuels }\end{array}$ & $\begin{array}{c}\text { CP05 } \\
\text { Furnishings, } \\
\text { household } \\
\text { equipment and } \\
\text { routine } \\
\text { maintenance of } \\
\text { the house } \\
\end{array}$ & $\begin{array}{l}\text { CP06 } \\
\text { Health }\end{array}$ & $\begin{array}{c}\text { CP07 } \\
\text { Transport }\end{array}$ & $\begin{array}{c}\text { CP08 } \\
\text { Communications }\end{array}$ & $\begin{array}{c}\text { CP09 } \\
\text { Recreation } \\
\text { and culture }\end{array}$ & $\begin{array}{c}\text { CP10 } \\
\text { Education }\end{array}$ & $\begin{array}{c}\text { CP11 } \\
\text { Restaurants } \\
\text { and hotels }\end{array}$ & $\begin{array}{c}\text { CP12 } \\
\text { Miscellaneous } \\
\text { goods and } \\
\text { services }\end{array}$ \\
\hline 1995 & $4,018,972$ & 0.144 & 0.038 & 0.066 & 0.207 & 0.069 & 0.031 & 0.130 & 0.019 & 0.090 & 0.009 & 0.083 & 0.114 \\
\hline 1996 & $4,254,879$ & 0.142 & 0.038 & 0.065 & 0.209 & 0.068 & 0.031 & 0.133 & 0.020 & 0.091 & 0.009 & 0.083 & 0.111 \\
\hline 1997 & $4,493,308$ & 0.139 & 0.038 & 0.064 & 0.208 & 0.068 & 0.032 & 0.135 & 0.021 & 0.092 & 0.009 & 0.083 & 0.112 \\
\hline 1998 & $4,703,861$ & 0.136 & 0.037 & 0.064 & 0.207 & 0.067 & 0.032 & 0.135 & 0.022 & 0.094 & 0.009 & 0.084 & 0.111 \\
\hline 1999 & $4,950,402$ & 0.132 & 0.038 & 0.063 & 0.206 & 0.067 & 0.032 & 0.138 & 0.023 & 0.096 & 0.010 & 0.085 & 0.111 \\
\hline 2000 & $5,324,171$ & 0.130 & 0.037 & 0.062 & 0.205 & 0.067 & 0.031 & 0.137 & 0.025 & 0.096 & 0.010 & 0.087 & 0.114 \\
\hline 2001 & $5,538,782$ & 0.131 & 0.037 & 0.060 & 0.207 & 0.066 & 0.032 & 0.135 & 0.027 & 0.096 & 0.010 & 0.087 & 0.114 \\
\hline 2002 & $5,711,320$ & 0.131 & 0.038 & 0.060 & 0.209 & 0.064 & 0.033 & 0.134 & 0.027 & 0.096 & 0.010 & 0.087 & 0.111 \\
\hline 2003 & $5,795,987$ & 0.130 & 0.037 & 0.059 & 0.212 & 0.064 & 0.034 & 0.133 & 0.028 & 0.095 & 0.010 & 0.087 & 0.112 \\
\hline 2004 & $6,066,073$ & 0.128 & 0.036 & 0.057 & 0.213 & 0.063 & 0.035 & 0.134 & 0.028 & 0.095 & 0.010 & 0.088 & 0.113 \\
\hline 2005 & $6,343,599$ & 0.126 & 0.036 & 0.056 & 0.217 & 0.061 & 0.034 & 0.136 & 0.028 & 0.093 & 0.010 & 0.087 & 0.115 \\
\hline 2006 & $6,653,406$ & 0.125 & 0.035 & 0.056 & 0.219 & 0.061 & 0.033 & 0.137 & 0.028 & 0.093 & 0.010 & 0.087 & 0.117 \\
\hline 2007 & $6,969,449$ & 0.125 & 0.034 & 0.055 & 0.219 & 0.060 & 0.034 & 0.136 & 0.027 & 0.093 & 0.011 & 0.088 & 0.119 \\
\hline 2008 & $7,033,958$ & 0.128 & 0.034 & 0.054 & 0.226 & 0.059 & 0.035 & 0.134 & 0.027 & 0.091 & 0.011 & 0.086 & 0.114 \\
\hline 2009 & $6,731,426$ & 0.130 & 0.035 & 0.053 & 0.236 & 0.058 & 0.037 & 0.129 & 0.027 & 0.090 & 0.011 & 0.085 & 0.109 \\
\hline 2010 & $7,020,303$ & 0.129 & 0.035 & 0.053 & 0.238 & 0.058 & 0.037 & 0.128 & 0.027 & 0.089 & 0.011 & 0.085 & 0.109 \\
\hline 2011 & $7,232,962$ & 0.129 & 0.036 & 0.053 & 0.238 & 0.057 & 0.037 & 0.131 & 0.027 & 0.088 & 0.011 & 0.085 & 0.109 \\
\hline 2012 & $7,411,889$ & 0.130 & 0.036 & 0.052 & 0.241 & 0.056 & 0.037 & 0.130 & 0.026 & 0.087 & 0.011 & 0.085 & 0.108 \\
\hline
\end{tabular}


Table S3.2. Price indices for the $1995-2012$ period and for the EU-27 by COICOP 2 digit classification . Source: Eurostat ${ }^{6}$

\begin{tabular}{|c|c|c|c|c|c|c|c|c|c|c|c|c|}
\hline Year & $\begin{array}{c}\text { CP01 } \\
\text { Food and } \\
\text { non- } \\
\text { alcoholic } \\
\text { beverages }\end{array}$ & $\begin{array}{c}\text { CP02 } \\
\text { Alcoholic } \\
\text { beverages, } \\
\text { tobacco } \\
\text { and } \\
\text { narcotics }\end{array}$ & $\begin{array}{c}\text { CP03 } \\
\text { Clothing } \\
\text { and } \\
\text { footwear }\end{array}$ & $\begin{array}{c}\text { CP04 } \\
\text { Housing, } \\
\text { water, } \\
\text { electricity, } \\
\text { gas and } \\
\text { other } \\
\text { fuels } \\
\end{array}$ & $\begin{array}{c}\text { CP05 } \\
\text { Furnishings, } \\
\text { household } \\
\text { equipment } \\
\text { and routine } \\
\text { maintenance } \\
\text { of the house }\end{array}$ & $\begin{array}{l}\text { CP06 } \\
\text { Health }\end{array}$ & $\begin{array}{c}\text { CP07 } \\
\text { Transport }\end{array}$ & $\begin{array}{c}\text { CP08 } \\
\text { Communications }\end{array}$ & $\begin{array}{c}\text { CP09 } \\
\text { Recreation } \\
\text { and culture }\end{array}$ & $\begin{array}{c}\text { CP10 } \\
\text { Education }\end{array}$ & $\begin{array}{c}\text { CP11 } \\
\text { Restaurants } \\
\text { and hotels }\end{array}$ & $\begin{array}{c}\text { CP12 } \\
\text { Miscellaneous } \\
\text { goods and } \\
\text { services }\end{array}$ \\
\hline 1995 & 84.1 & 73.3 & 88.4 & 77.5 & 86.5 & 83.1 & 80.6 & 104.6 & 90.1 & 72 & 79.7 & 84.5 \\
\hline 1996 & 92.7 & 82.8 & 94.2 & 83.7 & 92.1 & 88.5 & 86.2 & 110.5 & 95.5 & 78.8 & 85.3 & 88.1 \\
\hline 1997 & 95.5 & 88.1 & 96.7 & 88 & 94.7 & 91.8 & 89.8 & 109.9 & 98.3 & 86.2 & 88.8 & 91.8 \\
\hline 1998 & 97 & 91.8 & 97.5 & 91 & 96 & 94.5 & 90.7 & 108.6 & 98.6 & 89.9 & 91.4 & 92.9 \\
\hline 1999 & 97.5 & 95.4 & 98.7 & 94.7 & 97.7 & 96.4 & 93.5 & 104.2 & 98.5 & 94.3 & 95.1 & 94.1 \\
\hline 2000 & 100 & 100 & 100 & 100 & 100 & 100 & 100 & 100 & 100 & 100 & 100 & 100 \\
\hline 2001 & 105.2 & 103.7 & 100.1 & 103.6 & 101.7 & 101.8 & 101.3 & 97 & 100.4 & 104.6 & 103.6 & 102 \\
\hline 2002 & 108.2 & 108.4 & 100.3 & 107.5 & 103.4 & 105.1 & 103 & 97.6 & 101.2 & 109 & 107.8 & 101.5 \\
\hline 2003 & 108.2 & 111.1 & 98.2 & 108.7 & 102.6 & 106 & 103.3 & 95.7 & 98.2 & 111.4 & 108.6 & 102.7 \\
\hline 2004 & 109.3 & 116.1 & 98 & 112.8 & 103.5 & 109.5 & 107.1 & 94.2 & 97.8 & 116.6 & 112.2 & 105.9 \\
\hline 2005 & 111.1 & 121 & 97 & 118.6 & 104.3 & 112.5 & 112.1 & 94 & 96.6 & 119.2 & 115.4 & 109.2 \\
\hline 2006 & 114.8 & 125.9 & 97.1 & 124.8 & 105.7 & 115.1 & 116.4 & 92.2 & 96.4 & 125.6 & 119.6 & 113.8 \\
\hline 2007 & 119.8 & 132.3 & 97.5 & 130.5 & 107.9 & 117.6 & 119.9 & 90.5 & 96 & 134.5 & 124 & 118.2 \\
\hline 2008 & 126.6 & 136.8 & 94.8 & 134.1 & 108.3 & 119.1 & 122.9 & 87.3 & 92.5 & 137.1 & 125.3 & 116.1 \\
\hline 2009 & 125.1 & 140.3 & 91.2 & 134.5 & 108.2 & 118.9 & 116.9 & 84.5 & 89.6 & 137.5 & 125.1 & 107.5 \\
\hline 2010 & 126.5 & 147.2 & 91.8 & 138.9 & 109.7 & 120.6 & 122.9 & 84.6 & 90.2 & 141.5 & 127.9 & 110.6 \\
\hline 2011 & 130.9 & 155.7 & 93.3 & 144 & 111.6 & 122.7 & 130.5 & 84.2 & 90.3 & 143.9 & 130.9 & 113.7 \\
\hline 2012 & 136.3 & 166.5 & 95.6 & 149.6 & 114.3 & 125.4 & 137.4 & 82.5 & 90.9 & 149.3 & 135.7 & 115.3 \\
\hline
\end{tabular}


Table S3.3. Coefficients of the AIDS model.

\begin{tabular}{|l|c|c|c|c|}
\hline Coefficient & Estimate & Std. Error & t value & Pr(>|t|) \\
\hline alpha 1 & 0.892 & 0.194 & 4.597 & 0.000 \\
\hline alpha 2 & 0.181 & 0.143 & 1.269 & 0.207 \\
\hline alpha 3 & -0.044 & 0.220 & -0.201 & 0.841 \\
\hline alpha 4 & 1.624 & 0.300 & 5.422 & 0.000 \\
\hline alpha 5 & -0.112 & 0.227 & -0.494 & 0.622 \\
\hline alpha 6 & -0.155 & 0.184 & -0.845 & 0.400 \\
\hline alpha 7 & -0.349 & 0.286 & -1.221 & 0.225 \\
\hline alpha 8 & -0.734 & 0.196 & -3.746 & 0.000 \\
\hline alpha 9 & -0.637 & 0.176 & -3.615 & 0.000 \\
\hline alpha 10 & 0.201 & 0.190 & 1.063 & 0.290 \\
\hline alpha 11 & -0.179 & 0.194 & -0.925 & 0.357 \\
\hline alpha 12 & 0.312 & 0.350 & 0.890 & 0.375 \\
\hline beta 1 & -0.070 & 0.018 & -3.925 & 0.000 \\
\hline beta 2 & -0.013 & 0.013 & -1.009 & 0.315 \\
\hline beta 3 & 0.010 & 0.020 & 0.479 & 0.633 \\
\hline beta 4 & -0.130 & 0.028 & -4.735 & 0.000 \\
\hline beta 5 & 0.016 & 0.021 & 0.785 & 0.434 \\
\hline beta 6 & 0.017 & 0.017 & 1.017 & 0.312 \\
\hline beta 7 & 0.045 & 0.026 & 1.700 & 0.092 \\
\hline beta 8 & 0.070 & 0.018 & 3.878 & 0.000 \\
\hline beta 9 & 0.067 & 0.016 & 4.153 & 0.000 \\
\hline beta 10 & -0.018 & 0.017 & -1.009 & 0.315 \\
\hline beta 11 & 0.024 & 0.018 & 1.370 & 0.174 \\
\hline beta 12 & -0.018 & 0.032 & -0.564 & 0.574 \\
\hline gamma 1 1 & 0.075 & 0.011 & 7.034 & 0.000 \\
\hline gamma 1 2 & -0.014 & 0.008 & -1.823 & 0.071 \\
\hline gamma 1 3 & -0.019 & 0.010 & -1.857 & 0.066 \\
\hline gamma 1 4 & -0.024 & 0.017 & -1.416 & 0.160 \\
\hline gamma 1 5 & 0.006 & 0.011 & 0.549 & 0.584 \\
\hline gamma 1 6 & 0.010 & 0.008 & 1.142 & 0.256 \\
\hline gamma 1 7 & -0.016 & 0.015 & -1.087 & 0.280 \\
\hline gamma 1 8 & -0.004 & 0.009 & -0.431 & 0.667 \\
\hline gamma 1 9 & -0.014 & 0.008 & -1.844 & 0.068 \\
\hline gamma 1 10 & -0.020 & 0.010 & -2.118 & 0.036 \\
\hline gamma 1 11 & 0.018 & 0.010 & 1.849 & 0.067 \\
\hline gamma 1 12 & 0.001 & 0.014 & 0.085 & 0.932 \\
\hline gamma 2 1 & -0.014 & 0.008 & -1.823 & 0.071 \\
\hline gamma 2 2 & 0.002 & 0.013 & 0.181 & 0.856 \\
\hline gamma 2 3 & -0.005 & 0.012 & -0.433 & 0.666 \\
\hline gamma 2 4 & -0.024 & 0.019 & -1.241 & 0.217 \\
\hline gamma 2 5 & 0.013 & 0.015 & 0.851 & 0.397 \\
\hline gamma 2 6 & 0.005 & 0.011 & 0.443 & 0.659 \\
\hline & & & & \\
\hline
\end{tabular}




\begin{tabular}{|c|c|c|c|c|}
\hline gamma 27 & 0.011 & 0.017 & 0.633 & 0.528 \\
\hline gamma 28 & -0.005 & 0.008 & -0.593 & 0.554 \\
\hline gamma 29 & 0.012 & 0.010 & 1.197 & 0.234 \\
\hline gamma 210 & 0.003 & 0.013 & 0.229 & 0.819 \\
\hline gamma 211 & 0.028 & 0.011 & 2.485 & 0.014 \\
\hline gamma 212 & -0.026 & 0.015 & -1.677 & 0.096 \\
\hline gamma 31 & -0.019 & 0.010 & -1.857 & 0.066 \\
\hline gamma 32 & -0.005 & 0.012 & -0.433 & 0.666 \\
\hline gamma 33 & -0.073 & 0.022 & -3.318 & 0.001 \\
\hline gamma 34 & -0.097 & 0.027 & -3.647 & 0.000 \\
\hline gamma 35 & 0.047 & 0.020 & 2.273 & 0.025 \\
\hline gamma 36 & -0.020 & 0.015 & -1.317 & 0.191 \\
\hline gamma 37 & 0.062 & 0.022 & 2.898 & 0.005 \\
\hline gamma 38 & 0.032 & 0.011 & 2.788 & 0.006 \\
\hline gamma 39 & 0.019 & 0.013 & 1.395 & 0.166 \\
\hline gamma 310 & 0.008 & 0.016 & 0.521 & 0.604 \\
\hline gamma 311 & 0.065 & 0.016 & 4.072 & 0.000 \\
\hline gamma 312 & -0.018 & 0.018 & -0.950 & 0.344 \\
\hline gamma 41 & -0.024 & 0.017 & -1.416 & 0.160 \\
\hline gamma 42 & -0.024 & 0.019 & -1.241 & 0.217 \\
\hline gamma 43 & -0.097 & 0.027 & -3.647 & 0.000 \\
\hline gamma 44 & 0.090 & 0.060 & 1.516 & 0.132 \\
\hline gamma 45 & 0.046 & 0.030 & 1.533 & 0.128 \\
\hline gamma 46 & -0.020 & 0.021 & -0.957 & 0.341 \\
\hline gamma 47 & 0.024 & 0.032 & 0.732 & 0.466 \\
\hline gamma 48 & 0.029 & 0.017 & 1.699 & 0.092 \\
\hline gamma 49 & -0.043 & 0.018 & -2.417 & 0.017 \\
\hline gamma 410 & 0.030 & 0.024 & 1.229 & 0.222 \\
\hline gamma 411 & 0.042 & 0.026 & 1.604 & 0.112 \\
\hline gamma 412 & -0.052 & 0.027 & -1.926 & 0.057 \\
\hline gamma 51 & 0.006 & 0.011 & 0.549 & 0.584 \\
\hline gamma 52 & 0.013 & 0.015 & 0.851 & 0.397 \\
\hline gamma 53 & 0.047 & 0.020 & 2.273 & 0.025 \\
\hline gamma 54 & 0.046 & 0.030 & 1.533 & 0.128 \\
\hline gamma 55 & 0.008 & 0.033 & 0.238 & 0.812 \\
\hline gamma 56 & 0.002 & 0.020 & 0.081 & 0.936 \\
\hline gamma 57 & -0.045 & 0.022 & -2.057 & 0.042 \\
\hline gamma 58 & -0.013 & 0.012 & -1.049 & 0.296 \\
\hline gamma 59 & 0.003 & 0.015 & 0.204 & 0.839 \\
\hline gamma 510 & -0.024 & 0.017 & -1.441 & 0.152 \\
\hline gamma 511 & -0.061 & 0.019 & -3.214 & 0.002 \\
\hline gamma 512 & 0.018 & 0.020 & 0.895 & 0.373 \\
\hline gamma 61 & 0.010 & 0.008 & 1.142 & 0.256 \\
\hline gamma 62 & 0.005 & 0.011 & 0.443 & 0.659 \\
\hline gamma 63 & -0.020 & 0.015 & -1.317 & 0.191 \\
\hline
\end{tabular}




\begin{tabular}{|c|c|c|c|c|}
\hline gamma 64 & -0.020 & 0.021 & -0.957 & 0.341 \\
\hline gamma 65 & 0.002 & 0.020 & 0.081 & 0.936 \\
\hline gamma 66 & 0.004 & 0.021 & 0.205 & 0.838 \\
\hline gamma 67 & 0.011 & 0.016 & 0.673 & 0.503 \\
\hline gamma 68 & 0.017 & 0.010 & 1.665 & 0.099 \\
\hline gamma 69 & -0.002 & 0.011 & -0.133 & 0.895 \\
\hline gamma 610 & 0.018 & 0.013 & 1.368 & 0.174 \\
\hline gamma 611 & 0.004 & 0.016 & 0.220 & 0.826 \\
\hline gamma 612 & -0.028 & 0.015 & -1.928 & 0.056 \\
\hline gamma 71 & -0.016 & 0.015 & -1.087 & 0.280 \\
\hline gamma 72 & 0.011 & 0.017 & 0.633 & 0.528 \\
\hline gamma 73 & 0.062 & 0.022 & 2.898 & 0.005 \\
\hline gamma 74 & 0.024 & 0.032 & 0.732 & 0.466 \\
\hline gamma 75 & -0.045 & 0.022 & -2.057 & 0.042 \\
\hline gamma 76 & 0.011 & 0.016 & 0.673 & 0.503 \\
\hline gamma 77 & 0.018 & 0.035 & 0.534 & 0.595 \\
\hline gamma 78 & -0.005 & 0.016 & -0.315 & 0.753 \\
\hline gamma 79 & -0.002 & 0.015 & -0.134 & 0.894 \\
\hline gamma 710 & -0.005 & 0.022 & -0.241 & 0.810 \\
\hline gamma 711 & -0.067 & 0.018 & -3.691 & 0.000 \\
\hline gamma 712 & 0.013 & 0.028 & 0.478 & 0.634 \\
\hline gamma 81 & -0.004 & 0.009 & -0.431 & 0.667 \\
\hline gamma 82 & -0.005 & 0.008 & -0.593 & 0.554 \\
\hline gamma 83 & 0.032 & 0.011 & 2.788 & 0.006 \\
\hline gamma 84 & 0.029 & 0.017 & 1.699 & 0.092 \\
\hline gamma 85 & -0.013 & 0.012 & -1.049 & 0.296 \\
\hline gamma 86 & 0.017 & 0.010 & 1.665 & 0.099 \\
\hline gamma 87 & -0.005 & 0.016 & -0.315 & 0.753 \\
\hline gamma 88 & -0.004 & 0.013 & -0.308 & 0.759 \\
\hline gamma 89 & 0.009 & 0.008 & 1.064 & 0.290 \\
\hline gamma 810 & -0.001 & 0.011 & -0.071 & 0.943 \\
\hline gamma 811 & -0.027 & 0.011 & -2.469 & 0.015 \\
\hline gamma 812 & -0.028 & 0.016 & -1.718 & 0.089 \\
\hline gamma 91 & -0.014 & 0.008 & -1.844 & 0.068 \\
\hline gamma 92 & 0.012 & 0.010 & 1.197 & 0.234 \\
\hline gamma 93 & 0.019 & 0.013 & 1.395 & 0.166 \\
\hline gamma 94 & -0.043 & 0.018 & -2.417 & 0.017 \\
\hline gamma 95 & 0.003 & 0.015 & 0.204 & 0.839 \\
\hline gamma 96 & -0.002 & 0.011 & -0.133 & 0.895 \\
\hline gamma 97 & -0.002 & 0.015 & -0.134 & 0.894 \\
\hline gamma 98 & 0.009 & 0.008 & 1.064 & 0.290 \\
\hline gamma 99 & 0.013 & 0.013 & 0.994 & 0.322 \\
\hline gamma 910 & 0.013 & 0.011 & 1.183 & 0.239 \\
\hline gamma 911 & 0.014 & 0.012 & 1.188 & 0.237 \\
\hline gamma 912 & -0.020 & 0.014 & -1.402 & 0.164 \\
\hline
\end{tabular}




\begin{tabular}{|l|c|c|c|c|}
\hline gamma 10 1 & -0.020 & 0.010 & -2.118 & 0.036 \\
\hline gamma 10 2 & 0.003 & 0.013 & 0.229 & 0.819 \\
\hline gamma 10 3 & 0.008 & 0.016 & 0.521 & 0.604 \\
\hline gamma 10 4 & 0.030 & 0.024 & 1.229 & 0.222 \\
\hline gamma 10 5 & -0.024 & 0.017 & -1.441 & 0.152 \\
\hline gamma 10 6 & 0.018 & 0.013 & 1.368 & 0.174 \\
\hline gamma 10 7 & -0.005 & 0.022 & -0.241 & 0.810 \\
\hline gamma 10 8 & -0.001 & 0.011 & -0.071 & 0.943 \\
\hline gamma 10 9 & 0.013 & 0.011 & 1.183 & 0.239 \\
\hline gamma 10 10 & 0.023 & 0.018 & 1.264 & 0.209 \\
\hline gamma 10 11 & -0.035 & 0.014 & -2.445 & 0.016 \\
\hline gamma 10 12 & -0.009 & 0.018 & -0.484 & 0.629 \\
\hline gamma 11 1 & 0.018 & 0.010 & 1.849 & 0.067 \\
\hline gamma 11 2 & 0.028 & 0.011 & 2.485 & 0.014 \\
\hline gamma 11 3 & 0.065 & 0.016 & 4.072 & 0.000 \\
\hline gamma 11 4 & 0.042 & 0.026 & 1.604 & 0.112 \\
\hline gamma 11 5 & -0.061 & 0.019 & -3.214 & 0.002 \\
\hline gamma 11 6 & 0.004 & 0.016 & 0.220 & 0.826 \\
\hline gamma 11 7 & -0.067 & 0.018 & -3.691 & 0.000 \\
\hline gamma 11 8 & -0.027 & 0.011 & -2.469 & 0.015 \\
\hline gamma 11 9 & 0.014 & 0.012 & 1.188 & 0.237 \\
\hline gamma 11 10 & -0.035 & 0.014 & -2.445 & 0.016 \\
\hline gamma 11 11 & -0.008 & 0.022 & -0.365 & 0.715 \\
\hline gamma 11 12 & 0.027 & 0.017 & 1.628 & 0.106 \\
\hline gamma 12 1 & 0.001 & 0.014 & 0.085 & 0.932 \\
\hline gamma 12 2 & -0.026 & 0.015 & -1.677 & 0.096 \\
\hline gamma 12 3 & -0.018 & 0.018 & -0.950 & 0.344 \\
\hline gamma 12 4 & -0.052 & 0.027 & -1.926 & 0.057 \\
\hline gamma 12 5 & 0.018 & 0.020 & 0.895 & 0.373 \\
\hline gamma 12 6 & -0.028 & 0.015 & -1.928 & 0.056 \\
\hline gamma 12 7 & 0.013 & 0.028 & 0.478 & 0.634 \\
\hline gamma 12 8 & -0.028 & 0.016 & -1.718 & 0.089 \\
\hline gamma 12 9 & -0.020 & 0.014 & -1.402 & 0.164 \\
\hline gamma 12 10 & -0.009 & 0.018 & -0.484 & 0.629 \\
\hline gamma 12 11 & 0.027 & 0.017 & 1.628 & 0.106 \\
\hline gamma 12 12 & 0.121 & 0.035 & 3.423 & 0.001 \\
\hline
\end{tabular}


Table S3.4. Yearly marginal budget shares of the AIDS model.

\begin{tabular}{|c|c|c|c|c|c|c|c|c|c|c|c|c|}
\hline Year & $\begin{array}{c}\text { CP01 } \\
\text { Food and } \\
\text { non- } \\
\text { alcoholic } \\
\text { beverages }\end{array}$ & $\begin{array}{c}\text { CP02 } \\
\text { Alcoholic } \\
\text { beverages, } \\
\text { tobacco } \\
\text { and } \\
\text { narcotics }\end{array}$ & $\begin{array}{c}\text { CP03 } \\
\text { Clothing } \\
\text { and } \\
\text { footwear }\end{array}$ & $\begin{array}{c}\text { CP04 } \\
\text { Housing, } \\
\text { water, } \\
\text { electricity, } \\
\text { gas and } \\
\text { other } \\
\text { fuels }\end{array}$ & $\begin{array}{c}\text { CP05 } \\
\text { Furnishings, } \\
\text { household } \\
\text { equipment } \\
\text { and routine } \\
\text { maintenance } \\
\text { of the house }\end{array}$ & $\begin{array}{c}\text { CP06 } \\
\text { Health }\end{array}$ & $\begin{array}{c}\text { CP07 } \\
\text { Transport }\end{array}$ & $\begin{array}{c}\mathrm{CP08} \\
\text { Communications }\end{array}$ & $\begin{array}{c}\text { CP09 } \\
\text { Recreation } \\
\text { and } \\
\text { culture }\end{array}$ & $\begin{array}{c}\text { CP10 } \\
\text { Education }\end{array}$ & $\begin{array}{c}\text { CP11 } \\
\text { Restaurants } \\
\text { and hotels }\end{array}$ & $\begin{array}{c}\text { CP12 } \\
\text { Miscellaneous } \\
\text { goods and } \\
\text { services }\end{array}$ \\
\hline 1995 & 0.140 & 0.037 & 0.068 & 0.205 & 0.068 & 0.030 & 0.134 & 0.020 & 0.092 & 0.008 & 0.082 & 0.116 \\
\hline 1996 & 0.142 & 0.038 & 0.066 & 0.208 & 0.068 & 0.031 & 0.133 & 0.019 & 0.091 & 0.008 & 0.083 & 0.112 \\
\hline 1997 & 0.139 & 0.038 & 0.064 & 0.208 & 0.068 & 0.032 & 0.134 & 0.020 & 0.092 & 0.010 & 0.083 & 0.112 \\
\hline 1998 & 0.137 & 0.037 & 0.063 & 0.207 & 0.068 & 0.032 & 0.134 & 0.022 & 0.094 & 0.010 & 0.085 & 0.110 \\
\hline 1999 & 0.133 & 0.038 & 0.062 & 0.207 & 0.067 & 0.032 & 0.135 & 0.024 & 0.095 & 0.010 & 0.086 & 0.110 \\
\hline 2000 & 0.130 & 0.037 & 0.061 & 0.206 & 0.066 & 0.032 & 0.137 & 0.026 & 0.095 & 0.010 & 0.086 & 0.114 \\
\hline 2001 & 0.131 & 0.037 & 0.059 & 0.206 & 0.066 & 0.032 & 0.136 & 0.026 & 0.095 & 0.010 & 0.088 & 0.115 \\
\hline 2002 & 0.131 & 0.038 & 0.059 & 0.210 & 0.064 & 0.033 & 0.134 & 0.027 & 0.095 & 0.010 & 0.087 & 0.111 \\
\hline 2003 & 0.130 & 0.037 & 0.059 & 0.212 & 0.064 & 0.034 & 0.134 & 0.027 & 0.095 & 0.010 & 0.087 & 0.112 \\
\hline 2004 & 0.128 & 0.037 & 0.059 & 0.212 & 0.063 & 0.034 & 0.135 & 0.027 & 0.095 & 0.010 & 0.087 & 0.113 \\
\hline 2005 & 0.126 & 0.036 & 0.058 & 0.216 & 0.062 & 0.034 & 0.136 & 0.028 & 0.094 & 0.010 & 0.087 & 0.114 \\
\hline 2006 & 0.125 & 0.035 & 0.056 & 0.218 & 0.061 & 0.034 & 0.136 & 0.028 & 0.093 & 0.010 & 0.088 & 0.116 \\
\hline 2007 & 0.124 & 0.034 & 0.054 & 0.220 & 0.060 & 0.034 & 0.135 & 0.028 & 0.093 & 0.011 & 0.088 & 0.118 \\
\hline 2008 & 0.128 & 0.034 & 0.053 & 0.226 & 0.059 & 0.035 & 0.133 & 0.028 & 0.090 & 0.010 & 0.086 & 0.116 \\
\hline 2009 & 0.131 & 0.036 & 0.052 & 0.237 & 0.058 & 0.037 & 0.129 & 0.027 & 0.089 & 0.011 & 0.086 & 0.108 \\
\hline 2010 & 0.128 & 0.036 & 0.053 & 0.237 & 0.057 & 0.037 & 0.130 & 0.027 & 0.090 & 0.011 & 0.085 & 0.109 \\
\hline 2011 & 0.128 & 0.035 & 0.053 & 0.238 & 0.057 & 0.037 & 0.131 & 0.027 & 0.088 & 0.011 & 0.085 & 0.110 \\
\hline 2012 & 0.129 & 0.036 & 0.052 & 0.241 & 0.056 & 0.037 & 0.131 & 0.026 & 0.087 & 0.011 & 0.085 & 0.109 \\
\hline
\end{tabular}


Table S3.5. Yearly natural logarithm of the Stone's price index of the AIDS model.

0

1

2

3

4

5

6

7

8

9

10

11

12

13

\begin{tabular}{|l|l|}
\hline Year & InP \\
\hline $\mathbf{1 9 9 5}$ & 4.414 \\
\hline $\mathbf{1 9 9 6}$ & 4.485 \\
\hline $\mathbf{1 9 9 7}$ & 4.524 \\
\hline $\mathbf{1 9 9 8}$ & 4.543 \\
\hline $\mathbf{1 9 9 9}$ & 4.564 \\
\hline $\mathbf{2 0 0 0}$ & 4.605 \\
\hline $\mathbf{2 0 0 1}$ & 4.629 \\
\hline $\mathbf{2 0 0 2}$ & 4.651 \\
\hline $\mathbf{2 0 0 3}$ & 4.652 \\
\hline $\mathbf{2 0 0 4}$ & 4.675 \\
\hline $\mathbf{2 0 0 5}$ & 4.702 \\
\hline $\mathbf{2 0 0 6}$ & 4.733 \\
\hline $\mathbf{2 0 0 7}$ & 4.764 \\
\hline $\mathbf{2 0 0 8}$ & 4.777 \\
\hline $\mathbf{2 0 0 9}$ & 4.758 \\
\hline $\mathbf{2 0 1 0}$ & 4.784 \\
\hline $\mathbf{2 0 1 1}$ & 4.815 \\
\hline $\mathbf{2 0 1 2}$ & 4.848 \\
\hline
\end{tabular}


Supporting information S4: Results for the attributional life cycle assessment

Tables S4.1, S4.2 and S4.3 show the carbon dioxide emission inventory for the internal combustion engine, full battery electric and hydrogen fuel cell cars, respectively.

Table S4.1. Emission inventory and contribution by economic process for carbon dioxide emissions and for the internal combustion engine car (functional unit: $150,000 \mathrm{vkm}$, contribution $>=0.1 \%$ ).

\begin{tabular}{|c|c|c|}
\hline Process & $\begin{array}{l}\text { Value } \\
\text { (kg) }\end{array}$ & $\begin{array}{l}\text { Contribution } \\
(\%)\end{array}$ \\
\hline [P2741] operation, passenger car, petrol, EURO5[CH] & $1.41 \mathrm{E}+04$ & $40.52 \%$ \\
\hline [P2737] operation, passenger car, diesel, EURO5[CH] & $1.11 \mathrm{E}+04$ & $31.90 \%$ \\
\hline [P1504] refinery gas, burned in furnace[RER] & $1.10 \mathrm{E}+03$ & $3.16 \%$ \\
\hline [P1400] natural gas, sweet, burned in production flare[GLO] & 767 & $2.20 \%$ \\
\hline [P1490] heavy fuel oil, burned in refinery furnace[RER] & 510 & $1.47 \%$ \\
\hline [P997] lignite, burned in power plant[DE] & 343 & $0.99 \%$ \\
\hline [P1100] pig iron, at plant[GLO] & 313 & $0.90 \%$ \\
\hline [P851] hard coal, burned in power plant[DE] & 301 & $0.87 \%$ \\
\hline [P1329] natural gas, burned in industrial furnace $>100 \mathrm{~kW}[\mathrm{RER}]$ & 300 & $0.86 \%$ \\
\hline$[\mathrm{P} 1503]$ refinery gas, burned in furnace $[\mathrm{CH}]$ & 290 & $0.83 \%$ \\
\hline $\begin{array}{l}\text { [P1497] light fuel oil, burned in industrial furnace } 1 \mathrm{MW} \text {, non- } \\
\text { modulating[RER] }\end{array}$ & 271 & $0.78 \%$ \\
\hline [P1440] diesel, burned in diesel-electric generating set[GLO] & 228 & $0.66 \%$ \\
\hline [P823] hard coal, burned in industrial furnace 1-10MW[RER] & 205 & $0.59 \%$ \\
\hline [P1376] sweet gas, burned in gas turbine, production[NO] & 204 & $0.59 \%$ \\
\hline [P1820] operation, transoceanic tanker[OCE] & 190 & $0.55 \%$ \\
\hline [P1398] natural gas, sour, burned in production flare[GLO] & 187 & $0.54 \%$ \\
\hline [P1785] operation, lorry 20-28t, fleet average[CH] & 174 & $0.50 \%$ \\
\hline $\begin{array}{l}\text { [P1968] disposal, plastics, mixture, } 15.3 \% \text { water, to municipal } \\
\text { incineration[CH] }\end{array}$ & 153 & $0.44 \%$ \\
\hline [P854] hard coal, burned in power plant[PL] & 150 & $0.43 \%$ \\
\hline [P846] hard coal, burned in power plant[ES] & 146 & $0.42 \%$ \\
\hline $\begin{array}{l}\text { [P1485] heavy fuel oil, burned in industrial furnace } 1 \mathrm{MW} \text {, non- } \\
\text { modulating[RER] }\end{array}$ & 139 & $0.40 \%$ \\
\hline$[\mathrm{P} 480]$ clinker, at plant $[\mathrm{CH}]$ & 133 & $0.38 \%$ \\
\hline [P1006] lignite, burned in power plant[PL] & 128 & $0.37 \%$ \\
\hline [P2436] natural gas, burned in power plant[IT] & 126 & $0.36 \%$ \\
\hline [P1493] light fuel oil, burned in boiler 100kW, non-modulating[CH] & 108 & $0.31 \%$ \\
\hline [P1003] lignite, burned in power plant[CZ] & 107 & $0.31 \%$ \\
\hline [P553] diesel, burned in building machine[GLO] & 104 & $0.30 \%$ \\
\hline $\begin{array}{l}\text { [P1959] disposal, municipal solid waste, } 22.9 \% \text { water, to municipal } \\
\text { incineration[CH] }\end{array}$ & 102 & $0.29 \%$ \\
\hline $\begin{array}{l}{[\mathrm{P} 1325] \text { natural gas, burned in boiler fan burner low-NOx non- }} \\
\text { modulating }<100 \mathrm{~kW}[\mathrm{RER}]\end{array}$ & 100 & $0.29 \%$ \\
\hline [P1916] disposal, emulsion paint remains, $0 \%$ water, to hazardous waste & 97.3 & $0.28 \%$ \\
\hline
\end{tabular}




\begin{tabular}{|c|c|c|}
\hline incineration $[\mathrm{CH}]$ & & \\
\hline [P1668] butadiene, at plant[RER] & 96.7 & $0.28 \%$ \\
\hline $\begin{array}{l}\text { [P1977] disposal, rubber, unspecified, } 0 \% \text { water, to municipal } \\
\text { incineration }[\mathrm{CH}]\end{array}$ & 93.8 & $0.27 \%$ \\
\hline [P1489] heavy fuel oil, burned in refinery furnace[CH] & 93.5 & $0.27 \%$ \\
\hline [P1788] operation, lorry >16t, fleet average[RER] & 92.2 & $0.26 \%$ \\
\hline [P2373] heavy fuel oil, burned in power plant[IT] & 90.5 & $0.26 \%$ \\
\hline [P848] hard coal, burned in power plant[IT] & 84.8 & $0.24 \%$ \\
\hline [P1001] lignite, burned in power plant[GR] & 81.6 & $0.23 \%$ \\
\hline [P1114] sinter, iron, at plant[GLO] & 79.1 & $0.23 \%$ \\
\hline [P2446] blast furnace gas, burned in power plant[RER] & 71 & $0.20 \%$ \\
\hline [P1328] natural gas, burned in boiler modulating $>100 \mathrm{~kW}[\mathrm{RER}]$ & 70.6 & $0.20 \%$ \\
\hline [P999] lignite, burned in power plant[CS] & 62.1 & $0.18 \%$ \\
\hline [P1817] operation, barge tanker[RER] & 61.8 & $0.18 \%$ \\
\hline [P2438] natural gas, burned in power plant[NL] & 58.2 & $0.17 \%$ \\
\hline [P847] hard coal, burned in power plant[FR] & 58.1 & $0.17 \%$ \\
\hline [P2439] natural gas, burned in power plant[UCTE] & 55.5 & $0.16 \%$ \\
\hline $\begin{array}{l}\text { [P2166] electricity, at cogen ORC 1400kWth, wood, allocation } \\
\text { exergy[CH] }\end{array}$ & 55.1 & $0.16 \%$ \\
\hline [P2433] natural gas, burned in power plant[DE] & 54.8 & $0.16 \%$ \\
\hline $\begin{array}{l}\text { [P1330] natural gas, burned in industrial furnace low-NOx } \\
>100 \mathrm{~kW}[\mathrm{RER}]\end{array}$ & 54.2 & $0.16 \%$ \\
\hline [P2406] refinery gas, burned in flare[GLO] & 54.1 & $0.16 \%$ \\
\hline [P469] quicklime, in pieces, loose, at plant $[\mathrm{CH}]$ & 52.2 & $0.15 \%$ \\
\hline [P849] hard coal, burned in power plant[NL] & 51.8 & $0.15 \%$ \\
\hline [P1369] natural gas, burned in gas turbine, for compressor station[RU] & 50.1 & $0.14 \%$ \\
\hline [P260] carbon black, at plant[GLO] & 46.8 & $0.13 \%$ \\
\hline [P1819] operation, transoceanic freight ship[OCE] & 45.9 & $0.13 \%$ \\
\hline [P2434] natural gas, burned in power plant[ES] & 41.3 & $0.12 \%$ \\
\hline [P2369] heavy fuel oil, burned in power plant[ES] & 34.2 & $0.10 \%$ \\
\hline [P2441] natural gas, burned in power plant[CENTREL] & 34.2 & $0.10 \%$ \\
\hline Total & $3.48 E+04$ & \\
\hline Emissions per vkm $\left(b_{a}\right)(\mathrm{kg} / \mathrm{vkm})$ & 2.32E-01 & \\
\hline
\end{tabular}

Table S4.2. Emission inventory and contribution by economic process for carbon dioxide emissions and for the full battery electric car (functional unit: 150,000 vkm, contribution $>=0.1 \%$ ).

\begin{tabular}{|l|l|l|}
\hline Process & Value (kg) & $\begin{array}{l}\text { Contribution } \\
\mathbf{( \% )}\end{array}$ \\
\hline$[\mathrm{P} 997]$ lignite, burned in power plant[DE] & $2.11 \mathrm{E}+03$ & $11.34 \%$ \\
\hline$[\mathrm{P} 851]$ hard coal, burned in power plant[DE] & $1.63 \mathrm{E}+03$ & $8.76 \%$ \\
\hline$[\mathrm{P} 854]$ hard coal, burned in power plant[PL] & 986 & $5.30 \%$ \\
\hline$[\mathrm{P} 1006]$ lignite, burned in power plant[PL] & 840 & $4.51 \%$ \\
\hline$[\mathrm{P} 2436]$ natural gas, burned in power plant[IT] & 828 & $4.45 \%$ \\
\hline$[\mathrm{P} 846]$ hard coal, burned in power plant[ES] & 823 & $4.42 \%$ \\
\hline$[\mathrm{P} 1003]$ lignite, burned in power plant[CZ] & 699 & $3.76 \%$ \\
\hline
\end{tabular}




\begin{tabular}{|c|c|c|}
\hline [P1001] lignite, burned in power plant[GR] & 502 & $2.70 \%$ \\
\hline [P848] hard coal, burned in power plant[IT] & 496 & $2.67 \%$ \\
\hline [P2373] heavy fuel oil, burned in power plant[IT] & 472 & $2.54 \%$ \\
\hline [P2446] blast furnace gas, burned in power plant[RER] & 465 & $2.50 \%$ \\
\hline [P999] lignite, burned in power plant[CS] & 384 & $2.06 \%$ \\
\hline [P2438] natural gas, burned in power plant[NL] & 380 & $2.04 \%$ \\
\hline $\begin{array}{l}\text { [P2166] electricity, at cogen ORC 1400kWth, wood, allocation } \\
\text { exergy[CH] }\end{array}$ & 340 & $1.83 \%$ \\
\hline [P2433] natural gas, burned in power plant[DE] & 328 & $1.76 \%$ \\
\hline [P1329] natural gas, burned in industrial furnace $>100 \mathrm{~kW}[\mathrm{RER}]$ & 327 & $1.76 \%$ \\
\hline [P847] hard coal, burned in power plant[FR] & 314 & $1.69 \%$ \\
\hline [P849] hard coal, burned in power plant[NL] & 283 & $1.52 \%$ \\
\hline $\begin{array}{l}\text { [P1497] light fuel oil, burned in industrial furnace 1MW, non- } \\
\text { modulating[RER] }\end{array}$ & 283 & $1.52 \%$ \\
\hline [P2434] natural gas, burned in power plant[ES] & 271 & $1.46 \%$ \\
\hline [P823] hard coal, burned in industrial furnace 1-10MW[RER] & 236 & $1.27 \%$ \\
\hline [P2369] heavy fuel oil, burned in power plant[ES] & 224 & $1.20 \%$ \\
\hline [P2441] natural gas, burned in power plant[CENTREL] & 224 & $1.20 \%$ \\
\hline [P2439] natural gas, burned in power plant[UCTE] & 220 & $1.18 \%$ \\
\hline [P1100] pig iron, at plant[GLO] & 208 & $1.12 \%$ \\
\hline [P850] hard coal, burned in power plant[PT] & 172 & $0.92 \%$ \\
\hline [P1025] aluminium, primary, liquid, at plant[RER] & 159 & $0.85 \%$ \\
\hline [P1004] lignite, burned in power plant[HU] & 156 & $0.84 \%$ \\
\hline $\begin{array}{l}\text { [P1968] disposal, plastics, mixture, } 15.3 \% \text { water, to municipal } \\
\text { incineration[CH] }\end{array}$ & 152 & $0.82 \%$ \\
\hline [P1369] natural gas, burned in gas turbine, for compressor station[RU] & 143 & $0.77 \%$ \\
\hline [P998] lignite, burned in power plant[ES] & 135 & $0.73 \%$ \\
\hline [P480] clinker, at plant $[\mathrm{CH}]$ & 134 & $0.72 \%$ \\
\hline [P1819] operation, transoceanic freight ship[OCE] & 131 & $0.70 \%$ \\
\hline $\begin{array}{l}{[\mathrm{P} 1496] \text { light fuel oil, burned in industrial furnace } 1 \mathrm{MW} \text {, non- }} \\
\text { modulating }[\mathrm{CH}]\end{array}$ & 130 & $0.70 \%$ \\
\hline [P2432] natural gas, burned in power plant[BE] & 121 & $0.65 \%$ \\
\hline [P845] hard coal, burned in power plant[BE] & 118 & $0.63 \%$ \\
\hline [P2376] heavy fuel oil, burned in power plant[DE] & 109 & $0.59 \%$ \\
\hline [P1493] light fuel oil, burned in boiler 100kW, non-modulating[CH] & 104 & $0.56 \%$ \\
\hline $\begin{array}{l}\text { [P1959] disposal, municipal solid waste, } 22.9 \% \text { water, to municipal } \\
\text { incineration[CH] }\end{array}$ & 98.1 & $0.53 \%$ \\
\hline $\begin{array}{l}\text { [P1916] disposal, emulsion paint remains, } 0 \% \text { water, to hazardous } \\
\text { waste incineration }[\mathrm{CH}]\end{array}$ & 97.3 & $0.52 \%$ \\
\hline [P1002] lignite, burned in power plant[BA] & 96.3 & $0.52 \%$ \\
\hline [P2435] natural gas, burned in power plant[FR] & 96 & $0.52 \%$ \\
\hline $\begin{array}{l}{[\text { P1325] natural gas, burned in boiler fan burner low-NOx non- }} \\
\text { modulating }<100 \mathrm{~kW}[\mathrm{RER}]\end{array}$ & 95.7 & $0.51 \%$ \\
\hline $\begin{array}{l}\text { [P1977] disposal, rubber, unspecified, } 0 \% \text { water, to municipal } \\
\text { incineration }[\mathrm{CH}]\end{array}$ & 93.1 & $0.50 \%$ \\
\hline $\begin{array}{l}{[\mathrm{P} 1485] \text { heavy fuel oil, burned in industrial furnace } 1 \mathrm{MW} \text {, non- }} \\
\text { modulating[RER] }\end{array}$ & 88.9 & $0.48 \%$ \\
\hline [P852] hard coal, burned in power plant[CZ] & 75.6 & $0.41 \%$ \\
\hline
\end{tabular}




\begin{tabular}{|c|c|c|}
\hline [P553] diesel, burned in building machine[GLO] & 75.4 & $0.41 \%$ \\
\hline [P2431] natural gas, burned in power plant[AT] & 72.1 & $0.39 \%$ \\
\hline [P844] hard coal, burned in power plant[AT] & 71.9 & $0.39 \%$ \\
\hline [P1005] lignite, burned in power plant[MK] & 70.8 & $0.38 \%$ \\
\hline [P1504] refinery gas, burned in furnace[RER] & 70.1 & $0.38 \%$ \\
\hline [P1328] natural gas, burned in boiler modulating $>100 \mathrm{~kW}[\mathrm{RER}]$ & 69.7 & $0.37 \%$ \\
\hline [P2372] heavy fuel oil, burned in power plant[GR] & 68.1 & $0.37 \%$ \\
\hline $\begin{array}{l}\text { [P2669] electricity, at cogen with biogas engine, allocation } \\
\text { exergy[CH] }\end{array}$ & 65.9 & $0.35 \%$ \\
\hline [P1007] lignite, burned in power plant[SI] & 60.5 & $0.33 \%$ \\
\hline [P1114] sinter, iron, at plant[GLO] & 53.7 & $0.29 \%$ \\
\hline [P1008] lignite, burned in power plant[SK] & 52.6 & $0.28 \%$ \\
\hline [P2375] heavy fuel oil, burned in power plant[PT] & 50.6 & $0.27 \%$ \\
\hline [P469] quicklime, in pieces, loose, at plant [CH] & 48.9 & $0.26 \%$ \\
\hline [P1788] operation, lorry >16t, fleet average[RER] & 47.2 & $0.25 \%$ \\
\hline [P2445] coke oven gas, burned in power plant[RER] & 46.3 & $0.25 \%$ \\
\hline [P1400] natural gas, sweet, burned in production flare[GLO] & 44.8 & $0.24 \%$ \\
\hline [P1358] natural gas, burned in gas motor, for storage[DZ] & 44.2 & $0.24 \%$ \\
\hline [P2371] heavy fuel oil, burned in power plant[FR] & 42.9 & $0.23 \%$ \\
\hline [P1376] sweet gas, burned in gas turbine, production[NO] & 42 & $0.23 \%$ \\
\hline [P260] carbon black, at plant[GLO] & 39.5 & $0.21 \%$ \\
\hline [P1440] diesel, burned in diesel-electric generating set[GLO] & 37.3 & $0.20 \%$ \\
\hline [P855] hard coal, burned in power plant[SK] & 33.1 & $0.18 \%$ \\
\hline [P1490] heavy fuel oil, burned in refinery furnace[RER] & 32.4 & $0.17 \%$ \\
\hline [P2382] heavy fuel oil, burned in power plant[CZ] & 32.3 & $0.17 \%$ \\
\hline $\begin{array}{l}\text { [P1330] natural gas, burned in industrial furnace low-NOx } \\
>100 \mathrm{~kW}[\mathrm{RER}]\end{array}$ & 32.3 & $0.17 \%$ \\
\hline [P1010] SOx retained, in lignite flue gas desulphurisation[GLO] & 30.8 & $0.17 \%$ \\
\hline [P3844] natural gas, burned in power plant[US] & 30.7 & $0.16 \%$ \\
\hline [P1698] polyethylene, HDPE, granulate, at plant[RER] & 30.4 & $0.16 \%$ \\
\hline [P853] hard coal, burned in power plant[HR] & 29.2 & $0.16 \%$ \\
\hline [P2384] heavy fuel oil, burned in power plant[SK] & 28.9 & $0.16 \%$ \\
\hline [P1407] sweetening, natural gas[DE] & 27.8 & $0.15 \%$ \\
\hline $\begin{array}{l}\text { [P1917] disposal, hazardous waste, } 25 \% \text { water, to hazardous waste } \\
\text { incineration[CH] }\end{array}$ & 26.1 & $0.14 \%$ \\
\hline $\begin{array}{l}\text { [P1372] natural gas, burned in gas turbine, for compressor } \\
\text { station[UCTE] }\end{array}$ & 25.7 & $0.14 \%$ \\
\hline [P452] xylene, at plant[RER] & 24.7 & $0.13 \%$ \\
\hline $\begin{array}{l}\text { [P1921] disposal, solvents mixture, } 16.5 \% \text { water, to hazardous waste } \\
\text { incineration[CH] }\end{array}$ & 23 & $0.12 \%$ \\
\hline [P1398] natural gas, sour, burned in production flare[GLO] & 21.9 & $0.12 \%$ \\
\hline [P1367] natural gas, burned in gas turbine, for compressor station[DZ] & 21.7 & $0.12 \%$ \\
\hline [P2638] biogas, from biowaste, at storage[CH] & 21.3 & $0.11 \%$ \\
\hline [P2374] heavy fuel oil, burned in power plant[NL] & 19.3 & $0.10 \%$ \\
\hline [P1820] operation, transoceanic tanker[OCE] & 19 & $0.10 \%$ \\
\hline Total & $1.86 \mathrm{E}+04$ & \\
\hline
\end{tabular}


Emissions per vkm $\left(b_{p}\right)(k g / v k m)$

1.24E-01

24

Table S4.3. Emission inventory and contribution by economic process for carbon dioxide emissions and for the hydrogen fuel cell car (functional unit: 150,000 vkm, contribution $>=0.1 \%$ ).

\begin{tabular}{|c|c|c|}
\hline Process & $\begin{array}{l}\text { Value } \\
\text { (kg) }\end{array}$ & $\begin{array}{l}\text { Contribution } \\
(\%)\end{array}$ \\
\hline [P284] hydrogen, cracking, APME, at plant[RER] & $1.54 \mathrm{E}+03$ & $24.81 \%$ \\
\hline [P1329] natural gas, burned in industrial furnace $>100 \mathrm{~kW}[\mathrm{RER}]$ & 324 & $5.22 \%$ \\
\hline [P997] lignite, burned in power plant[DE] & 264 & $4.25 \%$ \\
\hline $\begin{array}{l}\text { [P1497] light fuel oil, burned in industrial furnace } 1 \mathrm{MW} \text {, non- } \\
\text { modulating[RER] }\end{array}$ & 251 & $4.04 \%$ \\
\hline [P851] hard coal, burned in power plant[DE] & 238 & $3.83 \%$ \\
\hline [P823] hard coal, burned in industrial furnace 1-10MW[RER] & 229 & $3.69 \%$ \\
\hline [P1100] pig iron, at plant[GLO] & 213 & $3.43 \%$ \\
\hline $\begin{array}{l}\text { [P1968] disposal, plastics, mixture, } 15.3 \% \text { water, to municipal } \\
\text { incineration[CH] }\end{array}$ & 152 & $2.45 \%$ \\
\hline [P846] hard coal, burned in power plant[ES] & 115 & $1.85 \%$ \\
\hline [P854] hard coal, burned in power plant[PL] & 115 & $1.85 \%$ \\
\hline$[\mathrm{P} 480]$ clinker, at plant $[\mathrm{CH}]$ & 111 & $1.79 \%$ \\
\hline [P1493] light fuel oil, burned in boiler 100kW, non-modulating[CH] & 102 & $1.64 \%$ \\
\hline [P1006] lignite, burned in power plant[PL] & 97.8 & $1.58 \%$ \\
\hline $\begin{array}{l}\text { [P1916] disposal, emulsion paint remains, } 0 \% \text { water, to hazardous waste } \\
\text { incineration[CH] }\end{array}$ & 97.3 & $1.57 \%$ \\
\hline [P2436] natural gas, burned in power plant[IT] & 96 & $1.55 \%$ \\
\hline $\begin{array}{l}{[\mathrm{P} 1325] \text { natural gas, burned in boiler fan burner low-NOx non- }} \\
\text { modulating }<100 \mathrm{~kW}[\mathrm{RER}]\end{array}$ & 95.7 & $1.54 \%$ \\
\hline $\begin{array}{l}\text { [P1977] disposal, rubber, unspecified, } 0 \% \text { water, to municipal } \\
\text { incineration[CH] }\end{array}$ & 93.2 & $1.50 \%$ \\
\hline [P1003] lignite, burned in power plant[CZ] & 81.4 & $1.31 \%$ \\
\hline $\begin{array}{l}\text { [P1959] disposal, municipal solid waste, } 22.9 \% \text { water, to municipal } \\
\text { incineration[CH] }\end{array}$ & 79.3 & $1.28 \%$ \\
\hline [P2373] heavy fuel oil, burned in power plant[IT] & 71 & $1.14 \%$ \\
\hline [P848] hard coal, burned in power plant[IT] & 66 & $1.06 \%$ \\
\hline [P1001] lignite, burned in power plant[GR] & 62.9 & $1.01 \%$ \\
\hline [P2446] blast furnace gas, burned in power plant[RER] & 54.4 & $0.88 \%$ \\
\hline [P1114] sinter, iron, at plant[GLO] & 53.7 & $0.86 \%$ \\
\hline [P1328] natural gas, burned in boiler modulating $>100 \mathrm{~kW}[\mathrm{RER}]$ & 50.1 & $0.81 \%$ \\
\hline [P999] lignite, burned in power plant[CS] & 47.8 & $0.77 \%$ \\
\hline [P2438] natural gas, burned in power plant[NL] & 46.3 & $0.75 \%$ \\
\hline [P847] hard coal, burned in power plant[FR] & 45.8 & $0.74 \%$ \\
\hline [P2439] natural gas, burned in power plant[UCTE] & 45.7 & $0.74 \%$ \\
\hline [P553] diesel, burned in building machine[GLO] & 45.4 & $0.73 \%$ \\
\hline $\begin{array}{l}\text { [P1485] heavy fuel oil, burned in industrial furnace 1MW, non- } \\
\text { modulating[RER] }\end{array}$ & 44.6 & $0.72 \%$ \\
\hline [P1369] natural gas, burned in gas turbine, for compressor station[RU] & 43.6 & $0.70 \%$ \\
\hline $\begin{array}{l}\text { [P2166] electricity, at cogen ORC 1400kWth, wood, allocation } \\
\text { exergy[CH] }\end{array}$ & 42.6 & $0.69 \%$ \\
\hline
\end{tabular}




\begin{tabular}{|c|c|c|}
\hline [P849] hard coal, burned in power plant[NL] & 42.1 & $0.68 \%$ \\
\hline [P2433] natural gas, burned in power plant[DE] & 40.8 & $0.66 \%$ \\
\hline $\begin{array}{l}\text { [P1330] natural gas, burned in industrial furnace low-NOx } \\
>100 \mathrm{~kW}[\mathrm{RER}]\end{array}$ & 40.6 & $0.65 \%$ \\
\hline [P260] carbon black, at plant[GLO] & 39.7 & $0.64 \%$ \\
\hline [P469] quicklime, in pieces, loose, at plant [CH] & 38.1 & $0.61 \%$ \\
\hline [P1788] operation, lorry $>16 t$, fleet average[RER] & 35.4 & $0.57 \%$ \\
\hline [P1819] operation, transoceanic freight ship[OCE] & 34.3 & $0.55 \%$ \\
\hline [P1674] ethylene, average, at plant[RER] & 32.1 & $0.52 \%$ \\
\hline [P2434] natural gas, burned in power plant[ES] & 31.4 & $0.51 \%$ \\
\hline [P3844] natural gas, burned in power plant[US] & 30.7 & $0.49 \%$ \\
\hline [P1504] refinery gas, burned in furnace[RER] & 28.4 & $0.46 \%$ \\
\hline [P2441] natural gas, burned in power plant[CENTREL] & 26.1 & $0.42 \%$ \\
\hline [P2369] heavy fuel oil, burned in power plant[ES] & 26 & $0.42 \%$ \\
\hline [P850] hard coal, burned in power plant[PT] & 24.7 & $0.40 \%$ \\
\hline $\begin{array}{l}\text { [P1917] disposal, hazardous waste, } 25 \% \text { water, to hazardous waste } \\
\text { incineration[CH] }\end{array}$ & 23.9 & $0.38 \%$ \\
\hline [P452] xylene, at plant[RER] & 21.1 & $0.34 \%$ \\
\hline [P1698] polyethylene, HDPE, granulate, at plant[RER] & 19.4 & $0.31 \%$ \\
\hline [P1400] natural gas, sweet, burned in production flare[GLO] & 18.9 & $0.30 \%$ \\
\hline [P998] lignite, burned in power plant[ES] & 18.4 & $0.30 \%$ \\
\hline [P1004] lignite, burned in power plant[HU] & 18.2 & $0.29 \%$ \\
\hline [P845] hard coal, burned in power plant[BE] & 18.1 & $0.29 \%$ \\
\hline [P1117] steel, converter, low-alloyed, at plant[RER] & 17.5 & $0.28 \%$ \\
\hline [P1025] aluminium, primary, liquid, at plant[RER] & 15.5 & $0.25 \%$ \\
\hline [P1703] polypropylene, granulate, at plant[RER] & 14.9 & $0.24 \%$ \\
\hline $\begin{array}{l}{[\mathrm{P} 1496] \text { light fuel oil, burned in industrial furnace } 1 \mathrm{MW} \text {, non- }} \\
\text { modulating }[\mathrm{CH}]\end{array}$ & 14.7 & $0.24 \%$ \\
\hline [P2432] natural gas, burned in power plant[BE] & 14.1 & $0.23 \%$ \\
\hline [P1376] sweet gas, burned in gas turbine, production[NO] & 13.2 & $0.21 \%$ \\
\hline [P1490] heavy fuel oil, burned in refinery furnace[RER] & 13.2 & $0.21 \%$ \\
\hline [P1002] lignite, burned in power plant[BA] & 12.7 & $0.20 \%$ \\
\hline [P2376] heavy fuel oil, burned in power plant[DE] & 12.7 & $0.20 \%$ \\
\hline [P790] flat glass, uncoated, at plant[RER] & 12.5 & $0.20 \%$ \\
\hline [P1440] diesel, burned in diesel-electric generating set[GLO] & 12.2 & $0.20 \%$ \\
\hline [P2435] natural gas, burned in power plant[FR] & 11.1 & $0.18 \%$ \\
\hline $\begin{array}{l}\text { [P1986] disposal, wood untreated, } 20 \% \text { water, to municipal } \\
\text { incineration }[\mathrm{CH}]\end{array}$ & 10.4 & $0.17 \%$ \\
\hline [P1358] natural gas, burned in gas motor, for storage[DZ] & 9.98 & $0.16 \%$ \\
\hline [P844] hard coal, burned in power plant[AT] & 9.64 & $0.16 \%$ \\
\hline [P1835] operation, freight train[RER] & 9.13 & $0.15 \%$ \\
\hline [P1005] lignite, burned in power plant[MK] & 8.98 & $0.14 \%$ \\
\hline [P852] hard coal, burned in power plant[CZ] & 8.8 & $0.14 \%$ \\
\hline [P815] hard coal coke, at plant[RER] & 8.76 & $0.14 \%$ \\
\hline [P1686] acrylonitrile-butadiene-styrene copolymer, ABS, at plant[RER] & 8.45 & $0.14 \%$ \\
\hline [P2669] electricity, at cogen with biogas engine, allocation exergy[CH] & 8.43 & $0.14 \%$ \\
\hline
\end{tabular}




\begin{tabular}{|c|c|c|}
\hline [P2431] natural gas, burned in power plant[AT] & 8.36 & $0.13 \%$ \\
\hline [P3871] hard coal, burned in power plant[SERC] & 8.31 & $0.13 \%$ \\
\hline [P3303] operation, lorry 16-32t, EURO5[RER] & 8.05 & $0.13 \%$ \\
\hline [P1820] operation, transoceanic tanker[OCE] & 7.95 & $0.13 \%$ \\
\hline [P1676] methylene diphenyl diisocyanate, at plant[RER] & 7.91 & $0.13 \%$ \\
\hline [P2372] heavy fuel oil, burned in power plant[GR] & 7.9 & $0.13 \%$ \\
\hline [P1785] operation, lorry $20-28 \mathrm{t}$, fleet average[CH] & 7.76 & $0.12 \%$ \\
\hline [P1007] lignite, burned in power plant[SI] & 7.57 & $0.12 \%$ \\
\hline [P1398] natural gas, sour, burned in production flare[GLO] & 7.51 & $0.12 \%$ \\
\hline $\begin{array}{l}\text { [P1372] natural gas, burned in gas turbine, for compressor } \\
\text { station[UCTE] }\end{array}$ & 7.43 & $0.12 \%$ \\
\hline [P1407] sweetening, natural gas[DE] & 7.42 & $0.12 \%$ \\
\hline [P3870] hard coal, burned in power plant[RFC] & 7.33 & $0.12 \%$ \\
\hline [P400] ethylene oxide, at plant[RER] & 7.08 & $0.11 \%$ \\
\hline [P352] titanium dioxide at plant, sulphate process, at plant[RER] & 7.04 & $0.11 \%$ \\
\hline [P822] hard coal coke, burned in stove 5-15kW[RER] & 6.94 & $0.11 \%$ \\
\hline $\begin{array}{l}\text { [P1922] disposal, used mineral oil, } 10 \% \text { water, to hazardous waste } \\
\text { incineration }[\mathrm{CH}]\end{array}$ & 6.41 & $0.10 \%$ \\
\hline [P353] titanium dioxide, chloride process, at plant[RER] & 6.26 & $0.10 \%$ \\
\hline [P1008] lignite, burned in power plant[SK] & 6.12 & $0.10 \%$ \\
\hline Total & $6.21 \mathrm{E}+03$ & \\
\hline Emissions per vkm $\left(b_{p}\right)(k g / v k m)$ & 4.14E-02 & \\
\hline
\end{tabular}


In this supporting information, we describe the method for input-output-based hybrid life cycle assessment. The method is described in the following five steps: monetarisation (S5.1), classification (S5.2), disaggregation (S5.3), final demand (S5.4) and emissions factors, direct emissions and additional emissions (S5.5).

\section{S5.1 Monetarisation}

36

37

Departing from the original life cycle inventory (LCI) described in supporting information S1, this first step involves converting this inventory from physical units, such as $\mathrm{kg}$ or $\mathrm{kWh}$, to monetary units. Joshi' $\mathrm{s}^{7}$ pioneering model for hybrid LCA proposes the use of cost sheets to this end, that is, a statement of all the costs incurred (materials, capital, labour and other expenses) during the manufacture of a product. However, this information is normally not publicly available, and, additionally, use stage and EoL data cannot be obtained directly through cost sheets. Alternatively, we derive unit values in euros ( $€$ per physical unit) from the Prodcom database ${ }^{6}$, which provides statistics on total value and volume sold on about 3,900 different types of manufactured products in the European Union. The unit values are derived from the last year available, and are not corrected for inflation. The flows from the end-of-life (EoL) stage are excluded to control for the fact that some of the EEIOTs used do not have waste management sectors (see section S5.5). For those products not present in Prodcom (e.g. fuel or electricity) or with different physical units than those from the LCI, additional assumptions and sources of information are used. Unit values for each physical flow and assumptions are described in table S5.1. Because the unit values are presented in euros, a yearly average currency exchange rate of 0.748 euro/dollar for the year $2011^{8}$ has been applied in the case of the WIOD. This exchange rate has also been used in the following steps where information on euros is presented. 
Table S5.1. Unit values for the monetarisation of the physical life cycle inventory and supporting information.

\begin{tabular}{|c|c|c|c|}
\hline Physical flow (as in Ecoinvent 2.2) & $\begin{array}{c}\text { Unit value } \\
(€ / \text { physical } \\
\text { unit })\end{array}$ & Prodcom product concordance & Comments \\
\hline Acetylene, at regional storehouse $[\mathrm{CH}]$ & 0.80 & $\begin{array}{l}\text { Unsaturated acyclic hydrocarbons (excluding ethylene, } \\
\text { propene, butene, buta-1.3-diene and isoprene) }\end{array}$ & \\
\hline $\begin{array}{l}\text { Alkyd paint, white, } 60 \% \text { in solvent, at } \\
\text { plant[RER] }\end{array}$ & 2.90 & $\begin{array}{l}\text { Other paints, varnishes dispersed or dissolved in an } \\
\text { aqueous medium }\end{array}$ & \\
\hline Aluminium alloy, $\mathrm{AlMg} 3$, at plant[RER] & 1.90 & $\begin{array}{l}\text { Unwrought aluminium alloys in primary form (excluding } \\
\text { aluminium powders and flakes) }\end{array}$ & \\
\hline $\begin{array}{l}\text { Aluminium, production mix, cast alloy, at } \\
\text { plant[RER] }\end{array}$ & 2.19 & Aluminium alloy plates, sheets and strips $>0.2 \mathrm{~mm}$ thick & \\
\hline $\begin{array}{l}\text { Aluminium, production mix, wrought alloy, at } \\
\text { plant[RER] }\end{array}$ & 2.19 & Aluminium alloy plates, sheets and strips $>0.2 \mathrm{~mm}$ thick & \\
\hline Barite, at plant[RER] & 0.17 & Sulphates of barium or aluminium & \\
\hline Boric oxide, at plant[GLO] & 0.39 & $\begin{array}{l}\text { Hydroxide and peroxide of magnesium oxides; hydroxides } \\
\text { and peroxides of strontium or barium }\end{array}$ & \\
\hline Carbon black, at plant[GLO] & 0.87 & Carbon (carbon blacks and other forms of carbon, n.e.c.) & \\
\hline Carbon dioxide liquid, at plant[RER] & 0.09 & Carbon dioxide & \\
\hline Cast iron, at plant[RER] & 1.22 & $\begin{array}{l}\text { Malleable iron castings for land vehicles, piston engines } \\
\text { and other machinery and mechanical appliances }\end{array}$ & \\
\hline Chemicals inorganic, at plant[GLO] & 1.30 & $\begin{array}{l}\text { Other inorganic bases; other metal oxides, hydroxides and } \\
\text { peroxides, n.e.c. }\end{array}$ & \\
\hline Chemicals organic, at plant[GLO] & 4.46 & Other organic compounds, n.e.c. & \\
\hline Chromium steel $18 / 8$, at plant[RER] & 1.11 & $\begin{array}{l}\text { Tinplate, other tinned sheet and strip, including } \\
\text { electrolytically chromium coated steel (ECCS) }\end{array}$ & \\
\hline
\end{tabular}




\begin{tabular}{|c|c|c|c|}
\hline Chromium, at regional storage[RER] & 26.16 & $\begin{array}{l}\text { Beryllium, chromium, germanium, vanadium, gallium, } \\
\text { hafnium ("celtium"), indium, niobium ("columbium"), } \\
\text { rhenium and thallium, and articles of these metals, n.e.c.; } \\
\text { waste and scrap of these metals (excluding of beryllium, } \\
\text { chromium and thallium) }\end{array}$ & \\
\hline $\begin{array}{l}\text { Cold impact extrusion, aluminium, } 3 \\
\text { strokes[RER] }\end{array}$ & 1.76 & Aluminium plates, sheets and strips $>0.2 \mathrm{~mm}$ thick & \\
\hline $\begin{array}{l}\text { Compressed air, average generation, }<30 \mathrm{~kW}, \\
12 \text { bar gauge, at compressor[RER] }\end{array}$ & 0.01 & Liquid air; compressed air & Conversion: $870 \mathrm{~kg} / \mathrm{m}^{3 \mathrm{a}}$ \\
\hline $\begin{array}{l}\text { Compressed air, average generation, }>30 \mathrm{~kW}, \\
6 \text { bar gauge, at compressor[RER] }\end{array}$ & 0.01 & Liquid air; compressed air & \\
\hline $\begin{array}{l}\text { Copper product manufacturing, average metal } \\
\text { working[RER] }\end{array}$ & 5.10 & $\begin{array}{l}\text { Copper and copper alloy plates, sheets and strip of a } \\
\text { thickness }>0.15 \mathrm{~mm} \text { (excluding expanded copper metal, } \\
\text { insulated electric strip) }\end{array}$ & \\
\hline Copper, at regional storage[RER] & 5.10 & $\begin{array}{l}\text { Copper and copper alloy plates, sheets and strip of a } \\
\text { thickness }>0.15 \mathrm{~mm} \text { (excluding expanded copper metal, } \\
\text { insulated electric strip) }\end{array}$ & \\
\hline Copper, primary, at refinery[ID] & 6.37 & $\begin{array}{l}\text { Unwrought unalloyed refined copper (excluding rolled, } \\
\text { extruded or forged sintered products) }\end{array}$ & \\
\hline Copper, secondary, at refinery[RER] & 5.10 & $\begin{array}{l}\text { Copper and copper alloy plates, sheets and strip of a } \\
\text { thickness }>0.15 \mathrm{~mm} \text { (excluding expanded copper metal, } \\
\text { insulated electric strip) }\end{array}$ & \\
\hline Degreasing, metal part in alkaline bath[RER] & 0.28 & Silicates; commercial alkali metal silicates & Conversion: $0.0015 \mathrm{~kg} / \mathrm{m}^{2 b}$ \\
\hline Dichloromethane, at plant[RER] & 0.61 & Dichloromethane (methylene chloride) & \\
\hline Diesel, at regional storage[CH] & 1.39 & & \multirow[t]{2}{*}{ https://www.energy.eu/fuelprices/ } \\
\hline Diesel, low-sulphur, at regional storage $[\mathrm{CH}]$ & 1.39 & & \\
\hline Dolomite, at plant[RER] & 0.01 & $\begin{array}{l}\text { Dolomite, crude, roughly trimmed or merely cut into } \\
\text { rectangular or square blocks or slabs (excluding calcined or } \\
\text { sintered dolomite, agglomerated dolomite and broken or } \\
\text { crushed dolomite for concrete aggregates, road metalling or } \\
\text { railway or other ballast) }\end{array}$ & \\
\hline Electricity $\operatorname{mix}[\mathrm{CH}]$ & 0.09 & & \multirow{3}{*}{$\begin{array}{l}\text { http://open-data.europa.eu/en/data/dataset/ } \\
\text { 7bcfa3f2-dd2c-4ed4-aff1-e7eadd961 ef6 }\end{array}$} \\
\hline Electricity, low voltage, at grid[CH] & 0.09 & & \\
\hline $\begin{array}{l}\text { Electricity, low voltage, production UCTE, at } \\
\text { grid[UCTE] }\end{array}$ & 0.09 & & \\
\hline
\end{tabular}




\begin{tabular}{|c|c|c|c|}
\hline Electricity, medium voltage, at grid[CH] & 0.09 & & \\
\hline $\begin{array}{l}\text { Electricity, medium voltage, production } \\
\text { UCTE, at grid[UCTE] }\end{array}$ & 0.09 & & \\
\hline Electronics for control units[RER] & 0.48 & $\begin{array}{l}\text { Electronic integrated circuits (excluding multichip } \\
\text { circuits): processors and controllers, whether or not } \\
\text { combined with memories, converters, logic circuits, } \\
\text { amplifiers, clock and timing circuits, or other circuits }\end{array}$ & Conversion: $0.002 \mathrm{~kg} / \mathrm{unit}^{\mathrm{d}}$ \\
\hline Epoxy resin, liquid, at plant[RER] & 1.98 & Epoxide resins, in primary forms & \\
\hline Ethylene glycol, at plant[RER] & 0.63 & Ethylene glycol (ethanediol) & \\
\hline Extrusion, plastic pipes[RER] & 2.65 & Rigid tubes, pipes and hoses of polymers of propylene & \\
\hline Ferrite, at plant[GLO] & 3.35 & Ferrite cores of transformers and inductors & \\
\hline Flat glass, uncoated, at plant[RER] & 3.45 & $\begin{array}{l}\text { Laminated safety glass, of size and shape suitable for } \\
\text { incorporation in motor vehicles, aircraft, spacecraft, vessels } \\
\text { and other vehicles }\end{array}$ & \\
\hline Glass fibre, at plant[RER] & 1.92 & $\begin{array}{l}\text { Other articles of glass fibre, of non-textile fibres, bulk, } \\
\text { flocks, others }\end{array}$ & \\
\hline Gold, at regional storage[RER] & $22,789.63$ & $\begin{array}{l}\text { Gold, in semi-manufactured forms for non-monetary use } \\
\text { (including plated with platinum) (excluding unwrought or } \\
\text { in powder form) }\end{array}$ & \\
\hline Graphite, at plant[RER] & 7.75 & $\begin{array}{l}\text { Articles of graphite or other carbon for electrical purposes } \\
\text { (excluding carbon electrodes and brushes) }\end{array}$ & \\
\hline $\begin{array}{l}\text { Heat, light fuel oil, at industrial furnace } \\
1 \mathrm{MW}[\mathrm{CH}]\end{array}$ & 0.01 & & \multirow{3}{*}{$\begin{array}{l}\text { http://ec.europa.eu/eurostat/ } \\
\text { documents/38154/41386/ } \\
\text { SEarticle2012S1.pdf/ } \\
\text { 364a98b3-648f-4716-b95a-71484f430a9f }\end{array}$} \\
\hline $\begin{array}{l}\text { Heat, natural gas, at boiler fan burner low- } \\
\text { NOx non-modulating }<100 \mathrm{~kW}[\mathrm{RER}]\end{array}$ & 0.01 & & \\
\hline $\begin{array}{l}\text { Heat, natural gas, at industrial furnace low- } \\
\text { NOx }>100 \mathrm{~kW} \text { RER] }\end{array}$ & 0.01 & & \\
\hline Hot rolling, steel[RER] & 0.97 & $\begin{array}{l}\text { Flat-rolled products of alloy steel other than stainless, of a } \\
\text { width }>=600 \mathrm{~mm} \text {, hot-rolled or cold-rolled "cold-reduced" } \\
\text { and further worked (excluding plated or coated with zinc } \\
\text { and products of silicon-electrical steel) }\end{array}$ & \\
\hline Hydrogen, liquid, at plant[RER] & 3.29 & Hydrogen & $\begin{array}{l}\text { Conversion: } 70.849 \mathrm{~kg} / \mathrm{m}^{3 \mathrm{~d}} \\
\mathrm{http}: / / \text { cordis.europa.eu } / \mathrm{result} / \mathrm{rcn} / \\
46874 \text { en.html }\end{array}$ \\
\hline Injection moulding[RER] & 5.61 & Moulded rubber articles for tractors and motor vehicles & \\
\hline Lead, at regional storage[RER] & 2.11 & Lead plates, sheets, strip and foil; lead powders and flakes & \\
\hline
\end{tabular}




\begin{tabular}{|c|c|c|c|}
\hline & & $\begin{array}{l}\text { (excluding lead powders or flakes prepared as colours; } \\
\text { paints or the like, insulated electric strip) }\end{array}$ & \\
\hline Lime, hydrated, packed, at plant $[\mathrm{CH}]$ & 0.08 & Quicklime & \\
\hline Lubricating oil, at plant[RER] & 1.86 & $\begin{array}{l}\text { Hydraulic brake fluids and other prepared liquids for } \\
\text { hydraulic transmission; not containing or containing }<70 \% \\
\text { by weight of petroleum oils or oils obtained from } \\
\text { bituminous mineral }\end{array}$ & \\
\hline $\begin{array}{l}\text { Magnesium-alloy, AZ91, diecasting, at } \\
\text { plant[RER] }\end{array}$ & 4.97 & $\begin{array}{l}\text { Magnesium and articles thereof (excluding waste and } \\
\text { scrap), n.e.c. }\end{array}$ & \\
\hline $\begin{array}{l}\text { Metal product manufacturing, average metal } \\
\text { working[RER] }\end{array}$ & 1.22 & $\begin{array}{l}\text { Malleable iron castings for land vehicles, piston engines } \\
\text { and other machinery and mechanical appliances }\end{array}$ & \\
\hline Methanol, at regional storage $[\mathrm{CH}]$ & 0.27 & Methanol (methyl alcohol) & \\
\hline Milling, aluminium, average[RER] & 5.48 & Articles of aluminium, n.e.c. & \\
\hline Milling, cast iron, average[RER] & 124.68 & $\begin{array}{l}\text { Malleable iron castings for land vehicles, piston engines } \\
\text { and other machinery and mechanical appliances }\end{array}$ & \\
\hline Milling, chromium steel, average[RER] & 124.68 & $\begin{array}{l}\text { Malleable iron castings for land vehicles, piston engines } \\
\text { and other machinery and mechanical appliances }\end{array}$ & \\
\hline Milling, steel, average[RER] & 124.68 & $\begin{array}{l}\text { Malleable iron castings for land vehicles, piston engines } \\
\text { and other machinery and mechanical appliances }\end{array}$ & \\
\hline Naphtha, at refinery[RER] & 0.67 & $\begin{array}{l}\text { Naphthalene and other aromatic hydrocarbon mixtures } \\
\text { (excluding benzole, toluole, xylole) }\end{array}$ & \\
\hline $\begin{array}{l}\text { Natural gas, burned in industrial furnace low- } \\
\text { NOx }>100 \mathrm{~kW}[\mathrm{RER}]\end{array}$ & 0.01 & & $\begin{array}{l}\text { http://ec.europa.eu/eurostat/ } \\
\text { documents/38154/41386/ } \\
\text { SEarticle2012S1.pdf/ } \\
\text { 364a98b3-648f-4716-b95a-71484f430a9f }\end{array}$ \\
\hline Neodymium oxide, at plant[CN] & 1.00 & $\begin{array}{l}\text { Alkali or alkaline-earth metals; rare-earth metals, scandium } \\
\text { and yttrium; mercury }\end{array}$ & \\
\hline Nickel, 99.5\%, at plant[GLO] & 19.55 & $\begin{array}{l}\text { Nickel and nickel alloy plate, sheet, strip and foil } \\
\text { (excluding expanded metal) }\end{array}$ & \\
\hline Nitrogen, liquid, at plant[RER] & 0.07 & Nitrogen & Conversion: $806.11 \mathrm{~kg} / \mathrm{m}^{3 \mathrm{~d}}$ \\
\hline Nylon 6, at plant[RER] & 4.30 & $\begin{array}{l}\text { Cordage, ropes or cables of polyethylene, polypropylene, } \\
\text { nylon or other polyamides or of polyesters measuring } \\
>50000 \text { decitex, of other synthetic fibres (excluding } \\
\text { binder or baler twine) }\end{array}$ & \\
\hline Nylon 66, at plant[RER] & 4.30 & $\begin{array}{l}\text { Cordage, ropes or cables of polyethylene, polypropylene, } \\
\text { nylon or other polyamides or of polyesters measuring } \\
>50000 \text { decitex, of other synthetic fibres (excluding }\end{array}$ & \\
\hline
\end{tabular}




\begin{tabular}{|c|c|c|c|}
\hline & & binder or baler twine) & \\
\hline Oxygen, liquid, at plant[RER] & 0.08 & Oxygen & Conversion: $1,141.2 \mathrm{~kg} / \mathrm{m}^{3 \mathrm{~d}}$ \\
\hline Palladium, at regional storage[RER] & $2,786.87$ & $\begin{array}{l}\text { Platinum, palladium, rhodium, iridium, osmium and } \\
\text { ruthenium, in semi-manufactured forms (excluding } \\
\text { unwrought or in powder form) }\end{array}$ & \\
\hline Paraffin, at plant[RER] & 0.64 & Paraformaldehyde & \\
\hline Petrol, low-sulphur, at regional storage $[\mathrm{CH}]$ & 1.48 & & \multirow[t]{2}{*}{ https://www.energy.eu/fuelprices/ } \\
\hline Petrol, unleaded, at regional storage $[\mathrm{CH}]$ & 1.48 & & \\
\hline Platinum, at regional storage[RER] & $2,786.87$ & $\begin{array}{l}\text { Platinum, palladium, rhodium, iridium, osmium and } \\
\text { ruthenium, in semi-manufactured forms (excluding } \\
\text { unwrought or in powder form) }\end{array}$ & \\
\hline Platinum, secondary, at refinery[RER] & $2,786.87$ & $\begin{array}{l}\text { Platinum, palladium, rhodium, iridium, osmium and } \\
\text { ruthenium, in semi-manufactured forms (excluding } \\
\text { unwrought or in powder form) }\end{array}$ & \\
\hline Plywood, outdoor use, at plant[RER] & 562.48 & $\begin{array}{l}\text { Plywood consisting solely of sheets of wood (excluding of } \\
\text { bamboo), each ply not exceeding } 6 \mathrm{~mm} \text { thickness, with at } \\
\text { least one outer ply of non-coniferous wood (excluding } \\
\text { trpical wood) }\end{array}$ & \\
\hline Polyester resin, unsaturated, at plant[RER] & 29.99 & Resinoids & \\
\hline $\begin{array}{l}\text { Polyethylene terephthalate, granulate, } \\
\text { amorphous, at plant[RER] }\end{array}$ & 1.34 & $\begin{array}{l}\text { Polyethylene terephthalate having a viscosity number of }>= \\
78 \mathrm{ml} / \mathrm{g}\end{array}$ & \\
\hline $\begin{array}{l}\text { Polyethylene terephthalate, granulate, bottle } \\
\text { grade, at plant[RER] }\end{array}$ & 1.34 & $\begin{array}{l}\text { Polyethylene terephthalate having a viscosity number of }>= \\
78 \mathrm{ml} / \mathrm{g}\end{array}$ & \\
\hline $\begin{array}{l}\text { Polyethylene, HDPE, granulate, at } \\
\text { plant[RER] }\end{array}$ & 1.23 & $\begin{array}{l}\text { Polyethylene having a specific gravity }<0.94 \text {, in primary } \\
\text { forms (excluding linear) }\end{array}$ & \\
\hline $\begin{array}{l}\text { Polymethyl methacrylate, beads, at } \\
\text { plant[RER] }\end{array}$ & 2.64 & Polymethyl methacrylate, in primary forms & \\
\hline Polypropylene, granulate, at plant[RER] & 1.11 & Polypropylene, in primary forms & \\
\hline $\begin{array}{l}\text { Polystyrene, high impact, HIPS, at } \\
\text { plant[RER] }\end{array}$ & 1.01 & $\begin{array}{l}\text { Polystyrene, in primary forms (excluding expansible } \\
\text { polystyrene) }\end{array}$ & \\
\hline Polyurethane, rigid foam, at plant[RER] & 2.43 & Polyurethanes, in primary forms & \\
\hline Powder coating, steel[RER] & 0.39 & $\begin{array}{l}\text { Granules and powders, of pig iron, spiegeleisen, iron or } \\
\text { steel }\end{array}$ & Conversion: $11.8 \mathrm{~m}^{2} / \mathrm{kg}^{\mathrm{e}}$ \\
\hline $\begin{array}{l}\text { Printed wiring board, surface mounted, } \\
\text { unspec., } \mathrm{Pb} \text { free, at plant[GLO] }\end{array}$ & 0.93 & Bare multilayer printed circuit boards & Conversion: $0.03917 \mathrm{~kg} / \mathrm{unit}^{\mathrm{f}}$ \\
\hline
\end{tabular}




\begin{tabular}{|c|c|c|c|}
\hline Propane/ butane, at refinery[CH] & 6.98 & $\begin{array}{l}\text { Mixtures containing halogenated derivatives of methane, } \\
\text { ethane or propane }\end{array}$ & \\
\hline Propylene glycol, liquid, at plant[RER] & 0.66 & Propylene glycol (propane-1.2-diol) & \\
\hline Reinforcing steel, at plant[RER] & 1.22 & Hot rolled wire rod, of bearing steel & \\
\hline Resistor, unspecified, at plant[GLO] & 5.60 & $\begin{array}{l}\text { Passive networks (including networks of resistors and/or } \\
\text { capacitors) (excluding resistor chip arrays, capacitor chip } \\
\text { arrays, boards containing active components, hybrids) }\end{array}$ & Conversion: $0.00094 \mathrm{~kg} /$ unit $^{\mathrm{g}}$ \\
\hline Rhodium, at regional storage[RER] & $2,786.87$ & $\begin{array}{l}\text { Platinum, palladium, rhodium, iridium, osmium and } \\
\text { ruthenium, in semi-manufactured forms (excluding } \\
\text { unwrought or in powder form) }\end{array}$ & \\
\hline Sanitary ceramics, at regional storage $[\mathrm{CH}]$ & 6.04 & $\begin{array}{l}\text { Refractory ceramic goods, n.e.c., by weight }>25 \% \text { graphite } \\
\text { or other forms of carbon }\end{array}$ & \\
\hline Secondary sulphur, at refinery[RER] & 0.06 & Sulphur, sublimed or precipitated; colloidal sulphur & \\
\hline Sheet rolling, aluminium[RER] & 1.76 & Aluminium plates, sheets and strips $>0.2 \mathrm{~mm}$ thick & \\
\hline Sheet rolling, steel[RER] & 0.97 & $\begin{array}{l}\text { Flat-rolled products of alloy steel other than stainless, of a } \\
\text { width }>=600 \mathrm{~mm} \text {, hot-rolled or cold-rolled "cold-reduced" } \\
\text { and further worked (excluding plated or coated with zinc } \\
\text { and products of silicon-electrical steel) }\end{array}$ & \\
\hline Silica sand, at plant[DE] & 1.38 & Silicon dioxide & \\
\hline Silicon, electronic grade, at plant[DE] & 9.15 & Silicon & \\
\hline Silver, at regional storage[RER] & 334.47 & $\begin{array}{l}\text { Silver, in semi-manufactured forms (including plated with } \\
\text { gold or platinum) (excluding unwrought or in powder } \\
\text { form) }\end{array}$ & \\
\hline $\begin{array}{l}\text { Silver, from combined gold-silver production, } \\
\text { at refinery[PE] }\end{array}$ & 334.47 & $\begin{array}{l}\text { Silver, in semi-manufactured forms (including plated with } \\
\text { gold or platinum) (excluding unwrought or in powder } \\
\text { form) }\end{array}$ & \\
\hline Sinter, iron, at plant[GLO] & 0.07 & Iron ores and concentrates (excluding roasted iron pyrites) & \\
\hline $\begin{array}{l}\text { Steel product manufacturing, average metal } \\
\text { working[RER] }\end{array}$ & 0.97 & $\begin{array}{l}\text { Flat-rolled products of alloy steel other than stainless, of a } \\
\text { width }>=600 \mathrm{~mm} \text {, hot-rolled or cold-rolled "cold-reduced" } \\
\text { and further worked (excluding plated or coated with zinc } \\
\text { and products of silicon-electrical steel) }\end{array}$ & \\
\hline Steel, converter, low-alloyed, at plant[RER] & 0.97 & $\begin{array}{l}\text { Flat-rolled products of alloy steel other than stainless, of a } \\
\text { width }>=600 \mathrm{~mm} \text {, hot-rolled or cold-rolled "cold-reduced" } \\
\text { and further worked (excluding plated or coated with zinc } \\
\text { and products of silicon-electrical steel) }\end{array}$ & \\
\hline Steel, electric, un- and low-alloyed, at & 0.62 & Flat-rolled products of alloy steel other than stainless, of a & \\
\hline
\end{tabular}




\begin{tabular}{|c|c|c|c|}
\hline plant[RER] & & $\begin{array}{l}\text { width of }<600 \mathrm{~mm} \text {, hot-rolled or cold-rolled "cold- } \\
\text { reduced" and electrolytically plated or coated with zinc } \\
\text { (excluding products of high-speed steel or silicon-electrical } \\
\text { steel) }\end{array}$ & \\
\hline Steel, low-alloyed, at plant[RER] & 0.69 & $\begin{array}{l}\text { Flat -rolled products of alloy steel other than stainless, of a } \\
\text { width of }<600 \mathrm{~mm} \text {, hot-rolled or cold-rolled "cold- } \\
\text { reduced" and plated or coated with zinc (excluding } \\
\text { electrolytically plated or coated, and products of high- } \\
\text { speed steel or silicon-electrical steel) }\end{array}$ & \\
\hline Sulphuric acid, liquid, at plant[RER] & 0.06 & Sulphuric acid & \\
\hline Synthetic rubber, at plant[RER] & 3.65 & Inner tubes, of rubber & \\
\hline Tap water, at user[RER] & 2.76 & & $\begin{array}{l}\text { http://www.eea.europa.eu/publications/assess } \\
\text { ment-of-full-cost-recovery/at_download/file }\end{array}$ \\
\hline Tempering, flat glass[RER] & 3.45 & $\begin{array}{l}\text { Laminated safety glass, of size and shape suitable for } \\
\text { incorporation in motor vehicles, aircraft, spacecraft, vessels } \\
\text { and other vehicles }\end{array}$ & \\
\hline Tetrafluoroethylene, at plant[RER] & 1.43 & $\begin{array}{l}\text { Polymers of ethylene, in primary forms (excluding } \\
\text { polyethylene, ethylene-vinyl acetate copolymers) }\end{array}$ & \\
\hline Thermoforming, with calendering[RER] & 4.57 & Other articles made from sheet & \\
\hline Tin, at regional storage[RER] & 15.99 & $\begin{array}{l}\text { Unwrought non-alloy tin (excluding tin powders and } \\
\text { flakes) }\end{array}$ & \\
\hline Transport, freight, rail[CH] & 0.12 & & \multirow{6}{*}{$\begin{array}{l}\text { http://ec.europa.eu/ten/transport/ } \\
\text { studies/doc/compete/compete_report_en.pdf }\end{array}$} \\
\hline Transport, lorry $>16 \mathrm{t}$, fleet average[RER] & 0.15 & & \\
\hline Transport, lorry 16-32t, EURO5[RER] & 0.15 & & \\
\hline Transport, lorry 3.5-20t, fleet average[CH] & 0.15 & & \\
\hline Transport, lorry 3.5-7.5t, EURO5[RER] & 0.15 & & \\
\hline Transport, transoceanic freight ship[OCE] & 0.15 & & \\
\hline Tube insulation, elastomere, at plant[DE] & 11.78 & $\begin{array}{l}\text { Insulating fittings of plastic, for electrical machines, } \\
\text { appliances or equipment (excluding electrical insulators) }\end{array}$ & \\
\hline Water, completely softened, at plant[RER] & 2.76 & & \\
\hline Wire drawing, copper[RER] & 1.04 & Wire rod for tyre cord & \\
\hline Wire drawing, steel[RER] & 1.04 & Wire rod for tyre cord & \\
\hline Zinc coating, coils[RER] & 3.57 & Articles of zinc, n.e.c. & \\
\hline Zinc coating, pieces[RER] & 3.57 & Articles of zinc, n.e.c. & Conversion: $0.04 \mathrm{~kg} / \mathrm{m}^{2 \mathrm{~h}}$ \\
\hline
\end{tabular}


${ }^{a}$ http://en.wikipedia.org/wiki/Liquid_air

${ }^{b}$ http://www.quakerchem.com/wp-content/uploads/2013/08/cs_quakerclean_BIO_1050_LH_cleaning.pdf

${ }^{\mathrm{c}} \mathrm{http}: / /$ www.nature.com/news/2002/021102/full/news021028-12.html

${ }^{\mathrm{d}} \mathrm{http}: / /$ encyclopedia.airliquide.com/encyclopedia.asp?LanguageID $=11$

e http://www.tiger-coatings.com/index.php?id=388\&L=2\&C=0\&tx_pressdownload_pi2\%5BshowUid $\% 5 \mathrm{D}=305$

${ }^{\mathrm{f}} \mathrm{http}: / / \mathrm{www} \cdot$ leiton.de/leiton-tools-weight-calculation.html

${ }^{\mathrm{g}} \mathrm{http} / / /$ www.taitroncomponents.com/catalog/Datasheet/RS5WF6030.pdf

${ }^{\mathrm{h}} \mathrm{http}: / / \mathrm{www}$. steelmillsoftheworld.com/activities/datacenter/G Note1.pdf 


\section{$63 \quad$ S5.2 Classification}

64 Once the physical LCI is converted to monetary units, the following step involves classifying the

65 physical flows according to the classification of each of the EEIOTs used. Table S5.2 describes the 66 concordances used to carry out this step. 
Table S5.2. Concordances between physical flows and Exiobase v2, WIOD and E3IOT classifications.

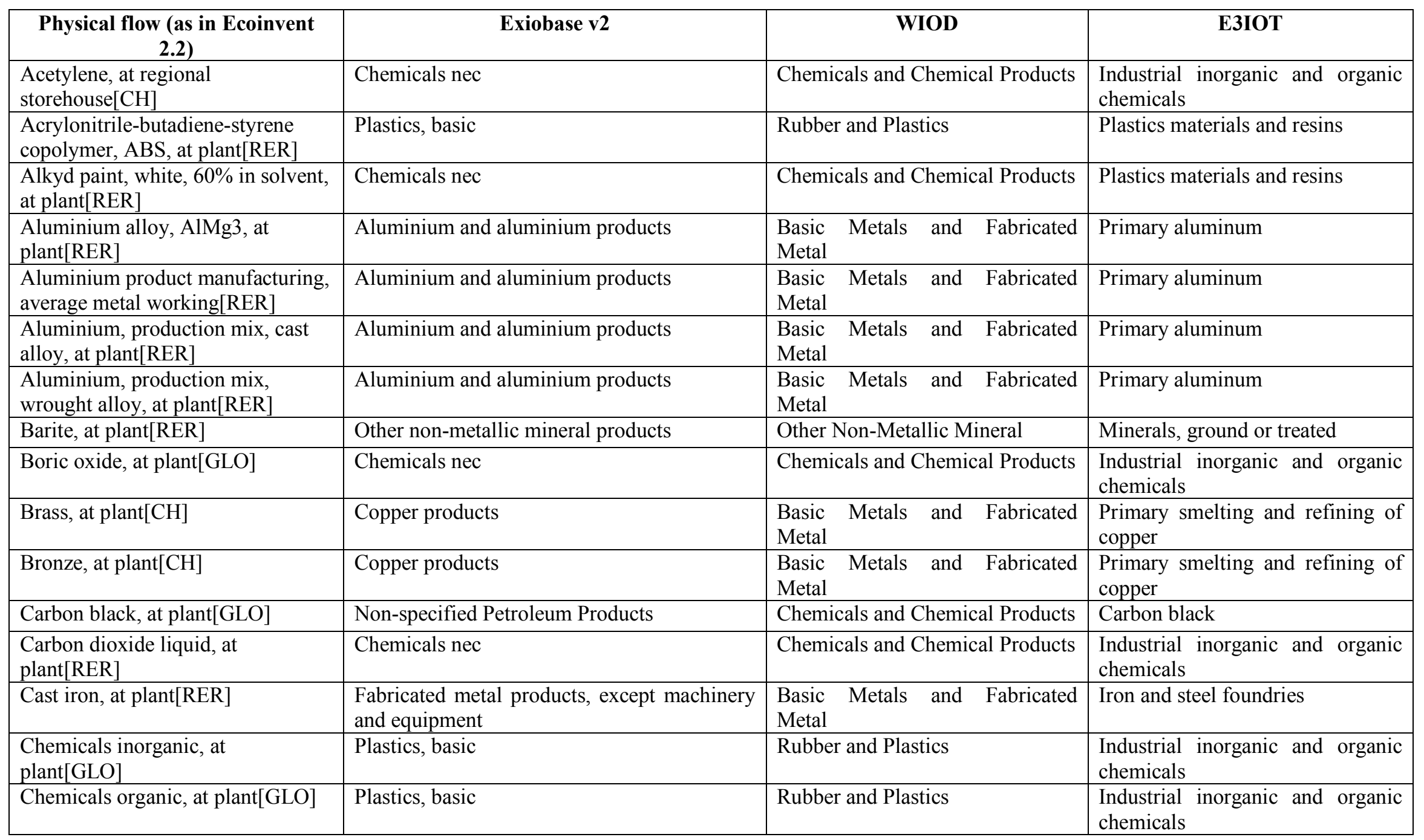




\begin{tabular}{|c|c|c|c|}
\hline $\begin{array}{l}\text { Chromium steel } 18 / 8 \text {, at } \\
\text { plant[RER] }\end{array}$ & $\begin{array}{l}\text { Basic iron and steel and of ferro-alloys and } \\
\text { first products thereof }\end{array}$ & $\begin{array}{lll}\text { Basic Metals and Fabricated } \\
\text { Metal }\end{array}$ & Blast furnaces and steel mills \\
\hline $\begin{array}{l}\text { Chromium, at regional } \\
\text { storage[RER] }\end{array}$ & Other non-metallic mineral products & Other Non-Metallic Mineral & Minerals, ground or treated \\
\hline $\begin{array}{l}\text { Cold impact extrusion, aluminium, } \\
3 \text { strokes[RER] }\end{array}$ & Aluminium and aluminium products & $\begin{array}{lll}\text { Basic Metals and Fabricated } \\
\text { Metal }\end{array}$ & Aluminum rolling and drawing \\
\hline $\begin{array}{l}\text { Compressed air, average } \\
\text { generation, }<30 \mathrm{~kW}, 12 \text { bar gauge, } \\
\text { at compressor[RER] }\end{array}$ & Machinery and equipment n.e.c. & Machinery, Nec & $\begin{array}{l}\text { General industrial machinery and } \\
\text { equipment, n.e.c. }\end{array}$ \\
\hline $\begin{array}{l}\text { Compressed air, average } \\
\text { generation, }>30 \mathrm{~kW}, 6 \text { bar gauge, at } \\
\text { compressor[RER] }\end{array}$ & Machinery and equipment n.e.c. & Machinery, Nec & $\begin{array}{l}\text { General industrial machinery and } \\
\text { equipment, n.e.c. }\end{array}$ \\
\hline $\begin{array}{l}\text { Copper product manufacturing, } \\
\text { average metal working[RER] }\end{array}$ & Copper products & $\begin{array}{lll}\text { Basic Metals and Fabricated } \\
\text { Metal }\end{array}$ & $\begin{array}{l}\text { Rolling, drawing, and extruding of } \\
\text { copper }\end{array}$ \\
\hline Copper, at regional storage[RER] & Copper products & $\begin{array}{llll}\text { Basic } & \text { Metals and Fabricated } \\
\text { Metal } & & & \\
\end{array}$ & $\begin{array}{l}\text { Primary smelting and refining of } \\
\text { copper }\end{array}$ \\
\hline Copper, primary, at refinery[ID] & Copper products & $\begin{array}{lll}\text { Basic } & \text { Metals and Fabricated } \\
\text { Metal } & & \\
\end{array}$ & $\begin{array}{l}\text { Primary smelting and refining of } \\
\text { copper }\end{array}$ \\
\hline $\begin{array}{l}\text { Copper, secondary, at } \\
\text { refinery[RER] }\end{array}$ & $\begin{array}{l}\text { Secondary copper for treatment, } \mathrm{Re}- \\
\text { processing of secondary copper into new } \\
\text { copper }\end{array}$ & Manufacturing, Nec; Recycling & $\begin{array}{l}\text { Primary smelting and refining of } \\
\text { copper }\end{array}$ \\
\hline $\begin{array}{l}\text { Degreasing, metal part in alkaline } \\
\text { bath[RER] }\end{array}$ & Chemicals nec & Chemicals and Chemical Products & $\begin{array}{l}\text { Industrial inorganic and organic } \\
\text { chemicals }\end{array}$ \\
\hline Dichloromethane, at plant[RER] & Chemicals nec & Chemicals and Chemical Products & $\begin{array}{l}\text { Industrial inorganic and organic } \\
\text { chemicals }\end{array}$ \\
\hline Diesel, at regional storage $[\mathrm{CH}]$ & Gas/Diesel Oil & $\begin{array}{l}\text { Sale, Maintenance and Repair of } \\
\text { Motor Vehicles and Motorcycles; } \\
\text { Retail Sale of Fuel }\end{array}$ & Petroleum refining \\
\hline $\begin{array}{l}\text { Diesel, low-sulphur, at regional } \\
\text { storage }[\mathrm{CH}]\end{array}$ & Gas/Diesel Oil & $\begin{array}{l}\text { Sale, Maintenance and Repair of } \\
\text { Motor Vehicles and Motorcycles; } \\
\text { Retail Sale of Fuel }\end{array}$ & Petroleum refining \\
\hline Dolomite, at plant[RER] & Other non-metallic mineral products & Other Non-Metallic Mineral & Minerals, ground or treated \\
\hline Electricity $\operatorname{mix}[\mathrm{CH}]$ & Electricity & Electricity, Gas and Water Supply & Electric services (utilities) \\
\hline Electricity, low voltage, at & Electricity & Electricity, Gas and Water Supply & Electricity \\
\hline
\end{tabular}




\begin{tabular}{|c|c|c|c|}
\hline $\operatorname{grid}[\mathrm{CH}]$ & & & \\
\hline $\begin{array}{l}\text { Electricity, low voltage, production } \\
\text { UCTE, at grid[UCTE] }\end{array}$ & Electricity & Electricity, Gas and Water Supply & Electricity \\
\hline $\begin{array}{l}\text { Electricity, medium voltage, at } \\
\text { grid }[\mathrm{CH}]\end{array}$ & Electricity & Electricity, Gas and Water Supply & Electric services (utilities) \\
\hline $\begin{array}{l}\text { Electricity, medium voltage, } \\
\text { production UCTE, at grid[UCTE] }\end{array}$ & Electricity & Electricity, Gas and Water Supply & Electricity \\
\hline Electronics for control units[RER] & Electrical machinery and apparatus n.e.c. & Electrical and Optical Equipment & Other electronic components \\
\hline Epoxy resin, liquid, at plant[RER] & Chemicals nec & Chemicals and Chemical Products & $\begin{array}{l}\text { Industrial inorganic and organic } \\
\text { chemicals }\end{array}$ \\
\hline Ethylene glycol, at plant[RER] & Chemicals nec & Chemicals and Chemical Products & $\begin{array}{l}\text { Industrial inorganic and organic } \\
\text { chemicals }\end{array}$ \\
\hline Extrusion, plastic pipes[RER] & Rubber and plastic products & Rubber and Plastics & $\begin{array}{l}\text { Miscellaneous plastics products, } \\
\text { n.e.c. }\end{array}$ \\
\hline Ferrite, at plant[GLO] & $\begin{array}{l}\text { Fabricated metal products, except machinery } \\
\text { and equipment }\end{array}$ & $\begin{array}{lll}\text { Basic Metals and Fabricated } \\
\text { Metal }\end{array}$ & $\begin{array}{l}\text { Iron and ferroalloy ores, and } \\
\text { miscellaneous metal ores, n.e.c. }\end{array}$ \\
\hline Flat glass, uncoated, at plant[RER] & Glass and glass products & Other Non-Metallic Mineral & $\begin{array}{l}\text { Glass and glass products, except } \\
\text { containers }\end{array}$ \\
\hline Glass fibre, at plant[RER] & Glass and glass products & Other Non-Metallic Mineral & $\begin{array}{l}\text { Glass and glass products, except } \\
\text { containers }\end{array}$ \\
\hline Gold, at regional storage[RER] & Precious metals & $\begin{array}{llll}\text { Basic } & \text { Metals and Fabricated } \\
\text { Metal } & & & \\
\end{array}$ & Jewelry, precious metal \\
\hline Graphite, at plant[RER] & Other non-metallic mineral products & Other Non-Metallic Mineral & $\begin{array}{l}\text { Nonferrous metal ores, except } \\
\text { copper }\end{array}$ \\
\hline $\begin{array}{l}\text { Heat, light fuel oil, at industrial } \\
\text { furnace } 1 \mathrm{MW}[\mathrm{CH}]\end{array}$ & Steam and hot water supply services & Electricity, Gas and Water Supply & Natural gas distribution \\
\hline $\begin{array}{l}\text { Heat, natural gas, at boiler fan } \\
\text { burner low-NOx non-modulating } \\
<100 \mathrm{~kW} \text { [RER] }\end{array}$ & Steam and hot water supply services & Electricity, Gas and Water Supply & Natural gas distribution \\
\hline $\begin{array}{l}\text { Heat, natural gas, at industrial } \\
\text { furnace low-NOx }>100 \mathrm{~kW}[\mathrm{RER}]\end{array}$ & Steam and hot water supply services & Electricity, Gas and Water Supply & Natural gas distribution \\
\hline Hot rolling, steel[RER] & $\begin{array}{l}\text { Basic iron and steel and of ferro-alloys and } \\
\text { first products thereof }\end{array}$ & $\begin{array}{lll}\text { Basic Metals and Fabricated } \\
\text { Metal }\end{array}$ & Blast furnaces and steel mills \\
\hline Hydrogen, liquid, at plant[RER] & Chemicals nec & Chemicals and Chemical Products & Industrial inorganic and organic \\
\hline
\end{tabular}




\begin{tabular}{|c|c|c|c|}
\hline & & & chemicals \\
\hline Injection moulding[RER] & Rubber and plastic products & Rubber and Plastics & $\begin{array}{l}\text { Miscellaneous plastics products, } \\
\text { n.e.c. }\end{array}$ \\
\hline Lead, at regional storage[RER] & Lead, zinc and tin and products thereof & $\begin{array}{llll}\text { Basic } & \text { Metals and Fabricated } \\
\text { Metal } & & & \\
\end{array}$ & Primary nonferrous metals, n.e.c. \\
\hline $\begin{array}{l}\text { Lime, hydrated, packed, at } \\
\text { plant }[\mathrm{CH}]\end{array}$ & Cement, lime and plaster & Other Non-Metallic Mineral & Cement, lime and plaster \\
\hline Lubricating oil, at plant[RER] & Lubricants & Chemicals and Chemical Products & Lubricating oils and greases \\
\hline $\begin{array}{l}\text { Magnesium-alloy, AZ91, } \\
\text { diecasting, at plant[RER] }\end{array}$ & Other non-metallic mineral products & Other Non-Metallic Mineral & $\begin{array}{l}\text { Iron and ferroalloy ores, and } \\
\text { miscellaneous metal ores, n.e.c. }\end{array}$ \\
\hline $\begin{array}{l}\text { Metal product manufacturing, } \\
\text { average metal working[RER] }\end{array}$ & $\begin{array}{l}\text { Fabricated metal products, except machinery } \\
\text { and equipment }\end{array}$ & $\begin{array}{llll}\text { Basic } & \text { Metals and Fabricated } \\
\text { Metal } & & & \\
\end{array}$ & Primary metal products, n.e.c. \\
\hline Methanol, at regional storage $[\mathrm{CH}]$ & Chemicals nec & Chemicals and Chemical Products & $\begin{array}{l}\text { Industrial inorganic and organic } \\
\text { chemicals }\end{array}$ \\
\hline Milling, aluminium, average[RER] & Aluminium and aluminium products & $\begin{array}{lll}\text { Basic Metals and Fabricated } \\
\text { Metal }\end{array}$ & Aluminum rolling and drawing \\
\hline Milling, cast iron, average[RER] & $\begin{array}{l}\text { Fabricated metal products, except machinery } \\
\text { and equipment }\end{array}$ & $\begin{array}{llll}\text { Basic } & \text { Metals and Fabricated } \\
\text { Metal } & & & \\
\end{array}$ & Iron and steel forgings \\
\hline $\begin{array}{l}\text { Milling, chromium steel, } \\
\text { average[RER] }\end{array}$ & $\begin{array}{l}\text { Fabricated metal products, except machinery } \\
\text { and equipment }\end{array}$ & $\begin{array}{llll}\text { Basic } & \text { Metals and } & \text { Fabricated } \\
\text { Metal } & & & \\
\end{array}$ & Iron and steel forgings \\
\hline Milling, steel, average[RER] & $\begin{array}{l}\text { Fabricated metal products, except machinery } \\
\text { and equipment }\end{array}$ & $\begin{array}{llll}\text { Basic } & \text { Metals and Fabricated } \\
\text { Metal } & & & \\
\end{array}$ & Iron and steel forgings \\
\hline Naphtha, at refinery[RER] & Naphtha & Chemicals and Chemical Products & $\begin{array}{l}\text { Products of petroleum and coal, } \\
\text { n.e.c. }\end{array}$ \\
\hline $\begin{array}{l}\text { Natural gas, burned in industrial } \\
\text { furnace low-NOx }>100 \mathrm{~kW}[\mathrm{RER}]\end{array}$ & $\begin{array}{l}\text { Distribution services of gaseous fuels through } \\
\text { mains }\end{array}$ & Electricity, Gas and Water Supply & Natural gas distribution \\
\hline Neodymium oxide, at plant[CN] & Precious metals & $\begin{array}{l}\text { Basic Metals and Fabricated } \\
\text { Metal }\end{array}$ & Jewelry, precious metal \\
\hline Nickel, 99.5\%, at plant[GLO] & Other non-ferrous metal products & $\begin{array}{lll}\text { Basic } & \text { Metals and Fabricated } \\
\text { Metal } & & \\
\end{array}$ & Primary nonferrous metals, n.e.c. \\
\hline Nitrogen, liquid, at plant[RER] & Chemicals nec & Chemicals and Chemical Products & $\begin{array}{l}\text { Industrial inorganic and organic } \\
\text { chemicals }\end{array}$ \\
\hline Nylon 6, at plant[RER] & Rubber and plastic products & Rubber and Plastics & $\begin{array}{l}\text { Miscellaneous plastics products, } \\
\text { n.e.c. }\end{array}$ \\
\hline
\end{tabular}




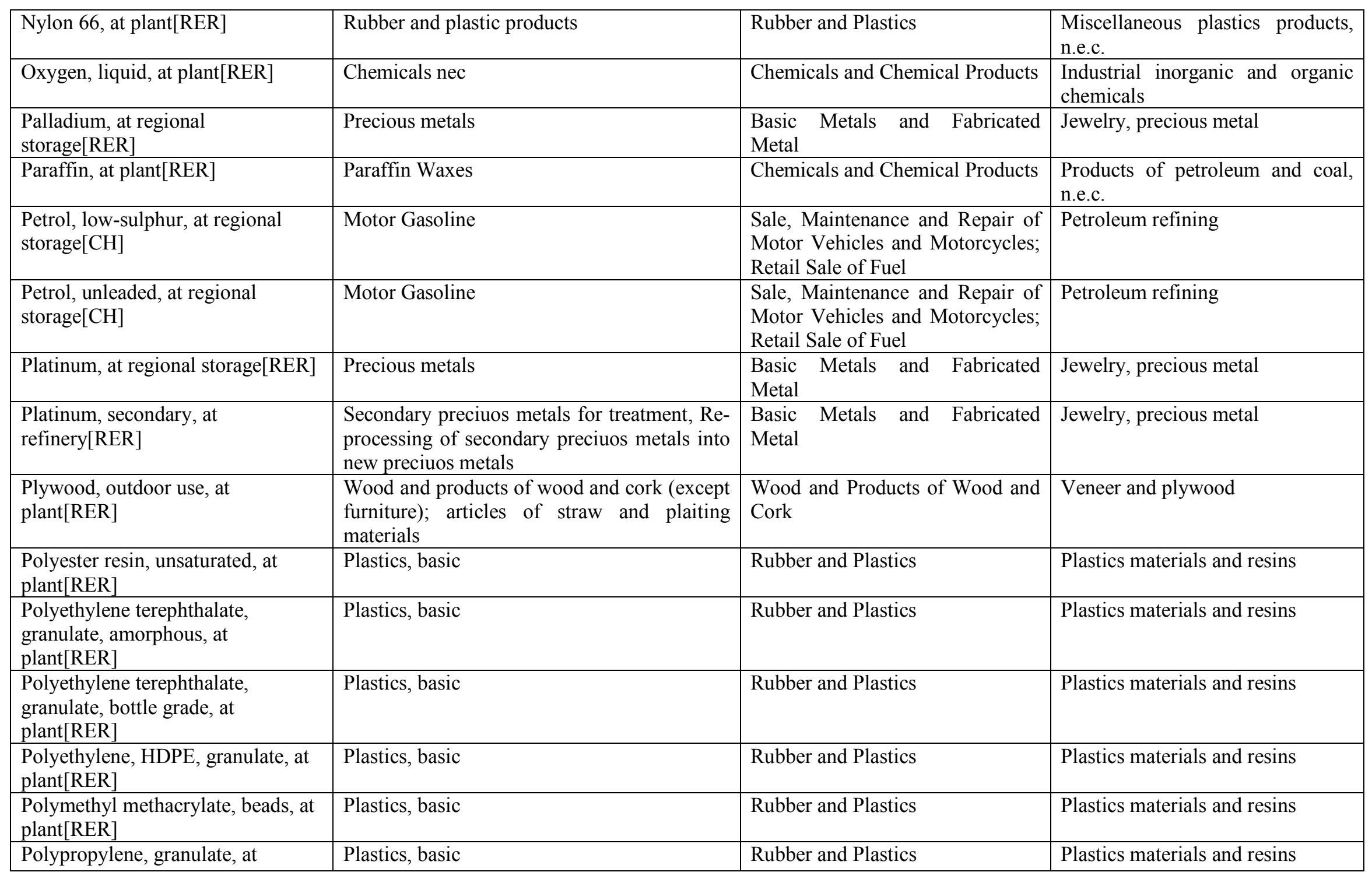




\begin{tabular}{|c|c|c|c|}
\hline plant[RER] & & & \\
\hline $\begin{array}{l}\text { Polystyrene, high impact, HIPS, at } \\
\text { plant[RER] }\end{array}$ & Plastics, basic & Rubber and Plastics & Plastics materials and resins \\
\hline $\begin{array}{l}\text { Polyurethane, rigid foam, at } \\
\text { plant[RER] }\end{array}$ & Plastics, basic & Rubber and Plastics & Plastics materials and resins \\
\hline Powder coating, steel[RER] & $\begin{array}{l}\text { Basic iron and steel and of ferro-alloys and } \\
\text { first products thereof }\end{array}$ & $\begin{array}{lll}\text { Basic } & \text { Metals and Fabricated } \\
\text { Metal } & & \\
\end{array}$ & Blast furnaces and steel mills \\
\hline $\begin{array}{l}\text { Printed wiring board, surface } \\
\text { mounted, unspec., Pb free, at } \\
\text { plant[GLO] }\end{array}$ & Electrical machinery and apparatus n.e.c. & Electrical and Optical Equipment & $\begin{array}{l}\text { Electrical machinery, equipment, } \\
\text { and supplies, n.e.c. }\end{array}$ \\
\hline Propane/ butane, at refinery $[\mathrm{CH}]$ & Non-specified Petroleum Products & Chemicals and Chemical Products & Petroleum refining \\
\hline $\begin{array}{l}\text { Propylene glycol, liquid, at } \\
\text { plant[RER] }\end{array}$ & Chemicals nec & Chemicals and Chemical Products & $\begin{array}{l}\text { Industrial inorganic and organic } \\
\text { chemicals }\end{array}$ \\
\hline Reinforcing steel, at plant[RER] & $\begin{array}{l}\text { Fabricated metal products, except machinery } \\
\text { and equipment }\end{array}$ & $\begin{array}{lll}\text { Basic Metals and Fabricated } \\
\text { Metal }\end{array}$ & Blast furnaces and steel mills \\
\hline $\begin{array}{l}\text { Resistor, unspecified, at } \\
\text { plant[GLO] }\end{array}$ & Electrical machinery and apparatus n.e.c. & Electrical and Optical Equipment & $\begin{array}{l}\text { Electrical machinery, equipment, } \\
\text { and supplies, n.e.c. }\end{array}$ \\
\hline Rhodium, at regional storage[RER] & Other non-metallic mineral products & Other Non-Metallic Mineral & $\begin{array}{l}\text { Industrial inorganic and organic } \\
\text { chemicals }\end{array}$ \\
\hline $\begin{array}{l}\text { Sanitary ceramics, at regional } \\
\text { storage }[\mathrm{CH}]\end{array}$ & Ceramic goods & Other Non-Metallic Mineral & $\begin{array}{l}\text { Clay, ceramic, and refractory } \\
\text { minerals }\end{array}$ \\
\hline $\begin{array}{l}\text { Secondary sulphur, at } \\
\text { refinery[RER] }\end{array}$ & Chemicals nec & Chemicals and Chemical Products & $\begin{array}{l}\text { Clay, ceramic, and refractory } \\
\text { minerals }\end{array}$ \\
\hline Sheet rolling, aluminium[RER] & Aluminium and aluminium products & $\begin{array}{llll}\text { Basic } & \text { Metals and Fabricated } \\
\text { Metal } & & \\
\end{array}$ & Aluminum rolling and drawing \\
\hline Sheet rolling, steel[RER] & $\begin{array}{l}\text { Basic iron and steel and of ferro-alloys and } \\
\text { first products thereof }\end{array}$ & $\begin{array}{llll}\text { Basic } & \text { Metals and Fabricated } \\
\text { Metal } & & & \\
\end{array}$ & Iron and steel forgings \\
\hline Silica sand, at plant[DE] & Other non-metallic mineral products & Other Non-Metallic Mineral & Minerals, ground or treated \\
\hline $\begin{array}{l}\text { Silicon, electronic grade, at } \\
\text { plant[DE] }\end{array}$ & Other non-metallic mineral products & Other Non-Metallic Mineral & Minerals, ground or treated \\
\hline Silver, at regional storage[RER] & Precious metals & $\begin{array}{lll}\text { Basic Metals and Fabricated } \\
\text { Metal }\end{array}$ & Jewelry, precious metal \\
\hline $\begin{array}{l}\text { Silver, from combined gold-silver } \\
\text { production, at refinery }[\mathrm{PE}]\end{array}$ & Precious metals & $\begin{array}{llll}\text { Basic } & \text { Metals and Fabricated } \\
\text { Metal } & & \\
\end{array}$ & Jewelry, precious metal \\
\hline
\end{tabular}




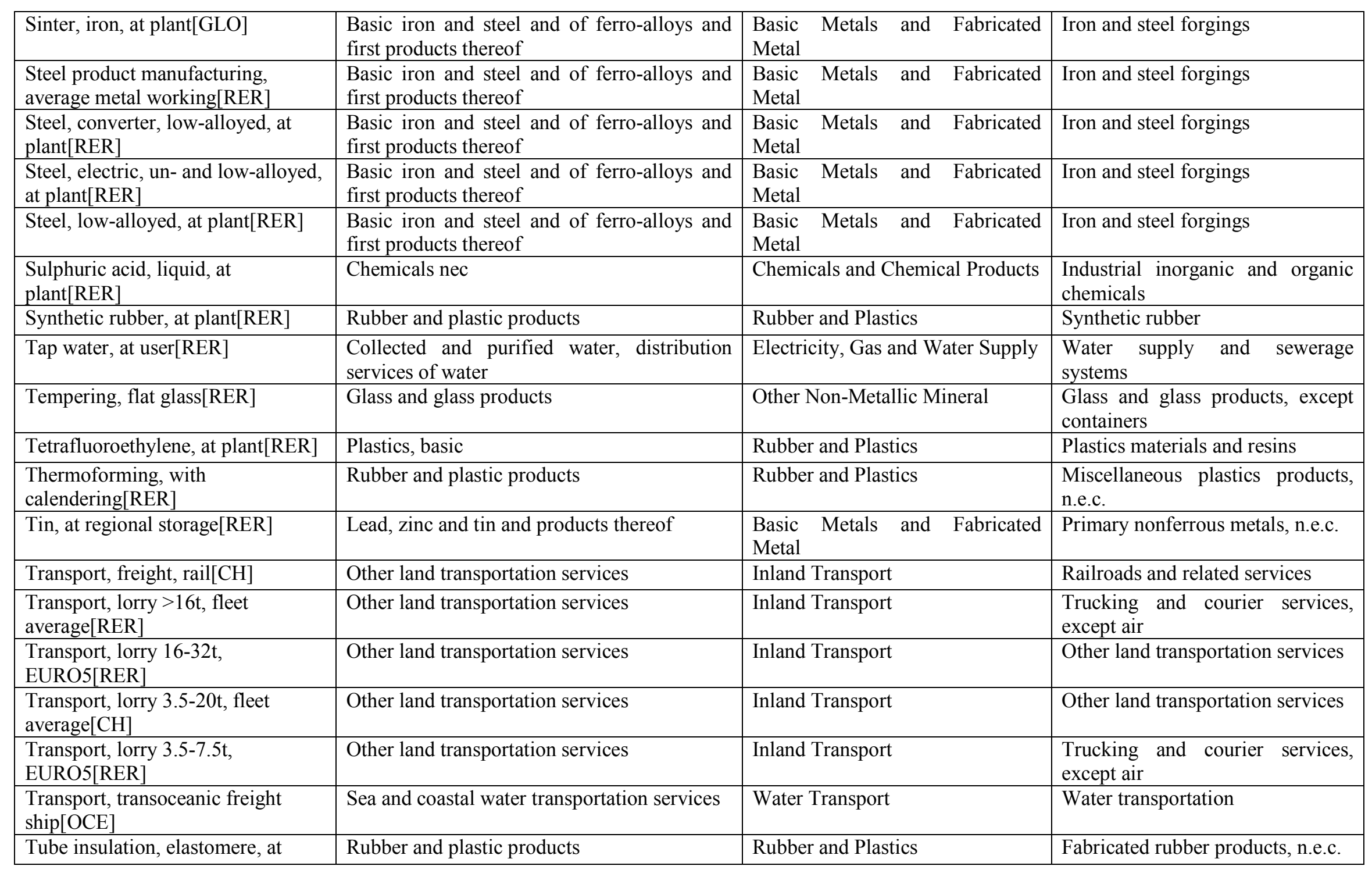




\begin{tabular}{|c|c|c|c|}
\hline plant[DE] & & & \\
\hline $\begin{array}{l}\text { Water, completely softened, at } \\
\text { plant[RER] }\end{array}$ & $\begin{array}{l}\text { Collected and purified water, distribution } \\
\text { services of water }\end{array}$ & Electricity, Gas and Water Supply & $\begin{array}{l}\text { Water supply and sewerage } \\
\text { systems }\end{array}$ \\
\hline Wire drawing, copper[RER] & Copper products & $\begin{array}{llll}\text { Basic } & \text { Metals and Fabricated } \\
\text { Metal } & & & \\
\end{array}$ & $\begin{array}{l}\text { Nonferrous wiredrawing and } \\
\text { insulating }\end{array}$ \\
\hline Wire drawing, steel[RER] & $\begin{array}{l}\text { Fabricated metal products, except machinery } \\
\text { and equipment }\end{array}$ & $\begin{array}{lll}\text { Basic } & \text { Metals and Fabricated } \\
\text { Metal } & & \\
\end{array}$ & $\begin{array}{l}\text { Nonferrous wiredrawing and } \\
\text { insulating }\end{array}$ \\
\hline Zinc coating, coils[RER] & Lead, zinc and tin and products thereof & $\begin{array}{l}\text { Basic Metals and Fabricated } \\
\text { Metal }\end{array}$ & Primary nonferrous metals, n.e.c. \\
\hline Zinc coating, pieces[RER] & Lead, zinc and tin and products thereof & $\begin{array}{l}\text { Basic Metals and Fabricated } \\
\text { Metal }\end{array}$ & Primary nonferrous metals, n.e.c. \\
\hline Zinc oxide, at plant[RER] & Lead, zinc and tin and products thereof & $\begin{array}{l}\text { Basic Metals and Fabricated } \\
\text { Metal }\end{array}$ & Primary nonferrous metals, n.e.c. \\
\hline $\begin{array}{l}\text { Zinc, primary, at regional } \\
\text { storage[RER] }\end{array}$ & Lead, zinc and tin and products thereof & $\begin{array}{lll}\text { Basic Metals and Fabricated } \\
\text { Metal }\end{array}$ & Primary nonferrous metals, n.e.c. \\
\hline
\end{tabular}




\section{S5.3 Disaggregation}

Following we describe the approach developed for disaggregating the inter-industry flow matrix $(\mathrm{Z})$ of a given EEIOT database. The objective is to disaggregate a sector so that specific technologies are represented in an EEIOT. In this case, the technologies are full-battery electric (FBE), hydrogen fuel cell (HFC) and internal combustion engine (ICE) passenger car technologies.

First, we identify the sector to be disaggregated (aggregated sector). In this case, "Manufacture of motor vehicles, trailers and semi-trailers" for Exiobase, "Transport Equipment" for WIOD and "Motor vehicles and passenger car bodies" for the E3IOT databases. Second, we construct the production input vector $\left(\vec{p}_{i}\right.$, columns in $\mathrm{Z}$ ) for each propulsion technology (electric [subscript $p$ ] and alternative [subscript $a$ ]) by multiplying the classified monetary LCIs $\left(\overrightarrow{L C I}_{m}\right)$ by the total amount of units $(s)$ produced of each technology in the EU27 (see table S5.3), as in equation S5.1.

$$
\vec{p}_{i, p \vee a}=\overrightarrow{L C I}_{m, p \vee a} S_{p \vee a}
$$

Table S5.3. Units produced for full battery electric, hydrogen fuel cell and internal combustion engine cars in the EU27.

\begin{tabular}{|l|r|l|}
\hline \multicolumn{1}{|c|}{ Technology } & $\begin{array}{c}\text { Units } \\
\text { produced }\end{array}$ & \multicolumn{1}{c|}{ Comments } \\
\hline Full battery electric & 87,840 & $\begin{array}{l}\text { Projected sales for the year 2015 (last year available). Based } \\
\text { on sales statistics gathered by EV Sales blog (http://ev- } \\
\text { sales.blogspot.nl/2015/05/europe-march-2015.html) }\end{array}$ \\
\hline Hydrogen fuel cell & 4,074 & $\begin{array}{l}\text { Estimated stock for the year 2015 (last year available) by } \\
\text { the European Commission }\end{array}$ \\
\hline $\begin{array}{l}\text { Internal combustion } \\
\text { engine }\end{array}$ & $17,383,144$ & $\begin{array}{l}\text { Production statistics for the year 2014 (last year available) } \\
\text { obtained from the OICA }{ }^{9}\end{array}$ \\
\hline
\end{tabular}

Next, and in the context of multi-regional (MR) EEIO databases (Exiobase and WIOD), we estimate how the total flows are distributed among the various producing countries by estimating production input weights $\left(\vec{w}_{i}\right)$. The production input weights are estimated proportionally to the existing aggregated sector, that is, production inputs will be required to the various countries in the same proportion as the aggregated sector. The weighted production input vector $\left(\vec{p}_{i, w}\right)$ by country (superscript $c$ ) is thus calculated as in equation S5.2.

$$
\vec{p}_{i, w, p \vee a}^{c}=\vec{p}_{i, p \vee a} \vec{w}_{i}^{c}
$$

In some cases, however, the required production inputs for a given country exceed the existing supply to the aggregated sector. In other words, more production inputs are required than what that country is currently supplying to the aggregated sector. To solve this issue, we apply a linear optimization algorithm. The purpose of this algorithm is to allocate those production inputs that exceed the actual supply to unconstrained supplying countries. By unconstrained supplying countries we mean those countries that, after satisfying the required inputs for each propulsion technology, have the largest stock available. The optimization problem is presented in equation S5.3, and the algorithm used to solve it is presented in equation S5.4.

$$
\text { Minimize: } \sum_{i=1}^{m}\left(R[i, j]-\sum_{k=1}^{m} A[k, i, j]\right)
$$

Subject to: $R[i, j] \geq 0 ; S[i, j] \geq 0$; 
112

$$
\sum_{k=1}^{m} A(k, i, j) \leq R[i, j] \text { and } \sum_{k=1}^{m} A(k, i, j) \leq S[i, j]
$$

Where $R$ is a $m x n$ matrix of $m$ countries and $n$ production inputs with the requirement of production input $j$ of country $i, S$ is a mxn matrix with the supply of production inputs and $A$ is a $m x n$ matrix containing the allocation of initially unallocated production inputs to country $k$.

$$
\begin{aligned}
& \forall i \in\left\{\vec{r}_{j}^{i}\right\} \\
& n r_{i}=r_{i}-s_{i} ; n r_{i}<0 \rightarrow n r_{i}=0 \\
& n s_{i}=s_{i}-r_{i} ; n s_{i}<0 \rightarrow n s_{i}=0 \\
& \sum_{i \in S} f(i) ; S=\left\{n r_{i}>0 \oplus n s_{i}>0\right\} \\
& r r_{i}=\min \left(\sum_{S} n r_{i}, \sum_{S} n s_{j}\right) ; j=\left\{n s_{i}==\max \left(n s_{i}\right)\right\} \\
& \Delta r_{i}=\frac{r r_{i} n r_{i}}{\sum r_{S} n r_{i}} \\
& \Delta s_{i}=\frac{r r_{i}}{\left|n s_{j}\right|} \\
& n r_{i}=n r_{i}-\Delta r_{i} \\
& n s_{i}=n s_{i}-\Delta s_{i} \\
& c r_{i}=\left(r_{i}-n r_{i}\right)+\Delta s_{i}
\end{aligned}
$$

Where $r$ is a $1 x(n x m)$ vector with the requirement of production inputs, $s$ is the supply capacity of production inputs and $\mathrm{cr}$ is the corrected requirement of production inputs once considering supply capacities.

Once the production recipes of all technologies are calculated, the production input vector of the rest of the aggregated sector $(r)$ is calculated following equation S5.4.

$$
\vec{p}_{i, r}^{c}=\vec{p}_{i, a g}^{c}-\vec{p}_{i, F B E}^{c}-\vec{p}_{i, H F C}^{c}-\vec{p}_{i, I C E}^{c}
$$

Where the subscript ag represents the original aggregated sector.

The next step involves disaggregating the production outputs $\left(\vec{p}_{o}\right.$, rows in Z). Due to lack of data, we assume that the production outputs of the new economic sectors (rest of aggregated sector, FBE, HFC and ICE cars) are allocated proportionally to the original aggregate sector. In other words, the allocation of production outputs to the different sectors and countries equals that of the original 
aggregated sector. We calculate the production outputs for each propulsion technology by multiplying the production output weights $\left(w_{o}^{c}\right)$ by the total output of each sector $(O)$, as in equation S5.5.

$$
\vec{p}_{o, w, p \vee a}^{c}=O_{p \vee a} \vec{w}_{o}^{c}
$$

$$
\text { With } O_{p \vee a}=z_{p \vee a} s_{p \vee a}
$$

Where $z$ is the purchasing price of each propulsion technology (see table S5.4).

Table S5.4. Purchasing price for full battery electric, hydrogen fuel cell and internal combustion engine cars in the EU27.

\begin{tabular}{|l|c|l|}
\hline \multicolumn{1}{|c|}{ Technology } & $\begin{array}{c}\text { Purchasin } \\
\text { g price (€) }\end{array}$ & \multicolumn{1}{|c|}{ Comments } \\
\hline Full battery electric & 28,900 & $\begin{array}{l}\text { For segment C/D and the year 2020 according to } \\
\text { McKensey and Company }\end{array}$ \\
\hline Hydrogen fuel cell & 30,900 & $\begin{array}{l}\text { For segment C/D and the year 2020 according to } \\
\text { McKensey and Company }\end{array}$ \\
\hline Internal combustion engine & 21,700 & $\begin{array}{l}\text { For segment C/D and the year 2020 according to } \\
\text { McKensey and Company } \\
\text { cars in the EU27 according to European Commission }\end{array}$ \\
\hline
\end{tabular}

Once the production output vectors of the propulsion technologies have been calculated, we recalculate the production output vector of the rest of the aggregated sector using equation S5.6.

$$
\vec{p}_{o, r}^{c}=\vec{p}_{o, a g}^{c}-\vec{p}_{o, F B E}^{c}-\vec{p}_{o, H F C}^{c}-\vec{p}_{o, I C E}^{c}
$$

Once both the production input and output vectors of the propulsion technologies and the rest of the aggregated sector have been calculated, these are used to replace the original aggregated sector in a given EEIOT.

\section{S5.4 Final demand}

The final demand for each propulsion technology will be made up of one vehicle and the corresponding fuel used during its life cycle (see table S5.5). In the context of MR-EEIOTs, final demand of both vehicles and fuels will take into consideration where are vehicles produced and used. Thus, we will calculate final demand weights for vehicles $\left(\vec{w}_{y, v}\right)$ and fuels $\left(\vec{w}_{y, f}\right)$ according to vehicle production and vehicle sales statistics, respectively (see table S5.6). Due to lack of data on HFC cars production, the weights for FBE cars have been used. Lastly, the weighted final demand $\left(\vec{y}_{w}\right)$ for vehicles $(v)$ and fuels $(f)$ is calculated by multiplying the final demand $(\mathrm{y})$ by the final demand weights as in equations S5.7 and S5.8.

$$
\vec{y}_{v, w, p \vee a}^{c}=y_{p \vee a} \vec{w}_{y, v}^{c}
$$

$$
\vec{y}_{f, w, p \vee a}^{c}=y_{p \vee a} \vec{w}_{y, f}^{c}
$$


Table S5.5. Vehicle and fuel final demand for full battery electric, hydrogen fuel cell and internal combustion engine cars in the EU27.

\begin{tabular}{|l|c|c|l|}
\hline Technology & $\begin{array}{c}\text { Vehicle } \\
(\boldsymbol{\epsilon})\end{array}$ & $\begin{array}{c}\text { Fuel } \\
(\boldsymbol{\epsilon})\end{array}$ & \multicolumn{1}{c|}{ Comments } \\
\hline $\begin{array}{l}\text { Full battery } \\
\text { electric }\end{array}$ & 28,900 & 3,672 & $\begin{array}{l}\text { Fuel sectors: "Distribution and trade of electricity" (Exiobase), } \\
\text { "Electricity, Gas and Water Supply" (WIOD) and "Electric } \\
\text { services (utilities)" (E3IOT). }\end{array}$ \\
\hline $\begin{array}{l}\text { Hydrogen } \\
\text { fuel cell }\end{array}$ & 30,900 & 4,315 & $\begin{array}{l}\text { Fuel sectors: "Chemicals nec" (Exiobase), "Chemicals and } \\
\text { Chemical Products" (WIOD) and "Industrial inorganic and } \\
\text { organic chemicals" (E3IOT). }\end{array}$ \\
\hline $\begin{array}{l}\text { Internal } \\
\text { combustion } \\
\text { engine }\end{array}$ & 21,700 & 10,697 & $\begin{array}{l}\text { Fuel sectors: "Retail sale of automotive fuel" (Exiobase), } \\
\text { "Sale, Maintenance and Repair of Motor Vehicles and } \\
\text { Motorcycles; Retail Sale of Fuel" (WIOD) and "Petroleum } \\
\text { refining" (E3IOT). }\end{array}$ \\
\hline
\end{tabular}

Table S5.6. Final demand weights for full-battery electric (FBE), hydrogen fuel cell (HFC) and internal combustion engine (ICE) passenger cars and fuels in the EU27. Sources: EV Sales blog (http://ev-sales.blogspot.nl/2015/05/europe-march-2015.html), OICA ${ }^{9}$ and ACEA ${ }^{10}$.

\begin{tabular}{|l|l|l|l|l|l|l|}
\hline Country & $\begin{array}{l}\text { FBE } \\
\text { car }\end{array}$ & $\begin{array}{l}\text { HFC } \\
\text { car }\end{array}$ & $\begin{array}{l}\text { ICE } \\
\text { car }\end{array}$ & $\begin{array}{l}\text { FBE } \\
\text { fuel }\end{array}$ & $\begin{array}{l}\text { HFC } \\
\text { fuel }\end{array}$ & $\begin{array}{l}\text { ICE } \\
\text { fuel }\end{array}$ \\
\hline Austria & 0.000 & 0.000 & 0.009 & 0.000 & 0.000 & 0.024 \\
\hline Belgium & 0.000 & 0.000 & 0.032 & 0.000 & 0.000 & 0.039 \\
\hline Finland & 0.000 & 0.000 & 0.003 & 0.000 & 0.000 & 0.008 \\
\hline France & 0.207 & 0.207 & 0.099 & 0.288 & 0.288 & 0.143 \\
\hline Germany & 0.262 & 0.262 & 0.370 & 0.152 & 0.152 & 0.242 \\
\hline Italy & 0.011 & 0.011 & 0.026 & 0.022 & 0.022 & 0.108 \\
\hline Portugal & 0.000 & 0.000 & 0.008 & 0.004 & 0.004 & 0.011 \\
\hline Spain & 0.060 & 0.060 & 0.125 & 0.021 & 0.021 & 0.068 \\
\hline Sweden & 0.000 & 0.000 & 0.010 & 0.031 & 0.031 & 0.024 \\
\hline Great Britain & 0.242 & 0.242 & 0.101 & 0.073 & 0.073 & 0.197 \\
\hline Netherlands & 0.180 & 0.180 & 0.000 & 0.394 & 0.394 & 0.031 \\
\hline Slovenia & 0.038 & 0.038 & 0.006 & 0.000 & 0.000 & 0.004 \\
\hline Czech Republic & 0.000 & 0.000 & 0.074 & 0.000 & 0.000 & 0.015 \\
\hline Hungary & 0.000 & 0.000 & 0.015 & 0.000 & 0.000 & 0.005 \\
\hline Poland & 0.000 & 0.000 & 0.031 & 0.000 & 0.000 & 0.026 \\
\hline Romania & 0.000 & 0.000 & 0.027 & 0.000 & 0.000 & 0.006 \\
\hline Slovakia & 0.000 & 0.000 & 0.064 & 0.000 & 0.000 & 0.006 \\
\hline Ireland & 0.000 & 0.000 & 0.000 & 0.001 & 0.001 & 0.008 \\
\hline Denmark & 0.000 & 0.000 & 0.000 & 0.011 & 0.011 & 0.015 \\
\hline Bulgaria & 0.000 & 0.000 & 0.000 & 0.000 & 0.000 & 0.002 \\
\hline Croatia & 0.000 & 0.000 & 0.000 & 0.000 & 0.000 & 0.003 \\
\hline Estonia & 0.000 & 0.000 & 0.000 & 0.000 & 0.000 & 0.002 \\
\hline Greece & 0.000 & 0.000 & 0.000 & 0.000 & 0.000 & 0.006 \\
\hline Latvia & 0.000 & 0.000 & 0.000 & 0.000 & 0.000 & 0.001 \\
\hline Lithuania & 0.000 & 0.000 & 0.000 & 0.000 & 0.000 & 0.001 \\
\hline Luxemburg & 0.000 & 0.000 & 0.000 & 0.000 & 0.000 & 0.004 \\
\hline Total & 1.000 & 1.000 & 1.000 & 1.000 & 1.000 & 1.000 \\
\hline
\end{tabular}


179

180 The emission factor matrix (F) is also disaggregated to describe the direct emissions from the 181 production of the newly added propulsion technologies. In this case, and following the initial LCI 182 described by Hawkins, Singh, Majeau-Bettez and Stromman ${ }^{1}$, we set the direct emissions to zero. 183 Furthermore, direct emissions from the final demand, that is, from the combustion of diesel and 184 gasoline, are calculated using attributional LCA, following the approach described in section 3.4.1. 185 Lastly, to the total emissions from production and use, we add life cycle emissions from the end-of-life 186 stage, also using the attributional LCA approach. This last step is justified by the generally poor 187 resolution of waste management sectors in the selected EEIOTs. 
The E3IOT database is an update of the CEDA EU25 model developed by CML and partners for the study of environmental impacts of products ${ }^{11,12}$. The E3IOT database is a detailed input output database for the EU25, with a resolution of 480 by 480 industries. It was constructed using the CEDA 3.0 model for the US ${ }^{13}$, by forcing European production structures on it from OECD input-output tables ( 35 by 35 industries). In addition, use and EoL stages are included by means of LCA databases and environmental accounts are completed using several different sources including LCA databases and national accounts. A detailed description of this database is available in Huppes and colleagues ${ }^{14}$. Relevant applications of the E3IOT table include the works of Tukker and colleagues ${ }^{15,16}$ and Wolf and colleagues ${ }^{17}$.

The world input-output database (WIOD) is a database containing multi-regional IOTs along with satellite accounts with environmental and socio-economic indicators for 41 national economies (40 countries plus rest of the world), from the period 1995 to 2011. It has a resolution of 35 industry sectors. It was developed in the context of the WIOD project, a project funded by the European Commission as part of the 7th Framework Programme. The database has been constructed using officially published IOTs in combination with national accounts data and international trade statistics, and combined this information with information on environmental extensions from various sources ${ }^{18}$. Additional information can be found in Timmer and colleagues ${ }^{19}$. Relevant works using the WIOD database include those from Koesler and Pothen ${ }^{20}$ and Boitier ${ }^{21}$.

The Exiobase database was developed within the EU-funded EXIOPOL and CREEA projects. The EXIOBASE version 2.2.1 used in this paper contains a multi-regional global IO database with highly detailed environmental satellite accounts (40 emitted substances, 15 types of land use, green and blue water use by water shed and 284 types of resources) as well as socio-economic data for 43 countries, 5 rest of continent regions aggregating all other countries, 163 industries and 200 products, with 2007 as the base year. In this study, the industry-by-industry classification has been used due to the fact that the factor input data needed to calculate the total output and the inter-industry flow matrix was only available at the industry level. The basic data is obtained through supply and use tables (SUT) and IOTs from Eurostat and other statistical offices, as well as official national accounts data and trade 217 statistics data. Further details can be found in Tukker and colleagues ${ }^{16}$ and Wood and colleagues ${ }^{22}$.

218 Some applications of the Exiobase database can be found in Tukker and colleagues ${ }^{23}$ and Schoer and 219 colleagues $^{24}$. 
Supporting information S7: Results of the two-way ANOVA test

221

222 Table S7.1. Result of the two-way ANOVA test. Significance codes: ' $* * *$ ' 0.001 , '**’ 0.01 , '*’ 0.05 , $223 \because 0.1, " 1$.

\begin{tabular}{|l|l|l|l|}
\hline \multicolumn{4}{|l|}{ Full battery electric car } \\
\hline & Degrees of freedom (df) & F-test value & p-value \\
\hline Method & 1 & 3.752 & 0.192 \\
\hline Database & 2 & 0.244 & 0.804 \\
\hline Hydrogen fuel cell car & 51.92 & $0.0187^{*}$ \\
\hline Method & 1 & 20.21 & $0.0471^{*}$ \\
\hline Database & 2 &
\end{tabular}

224 
1. Hawkins, T. R.; Singh, B.; Majeau-Bettez, G.; Stromman, A. H., Comparative Environmental Life Cycle Assessment of Conventional and Electric Vehicles. Journal of Industrial Ecology 2012, 17, (1).

$229 \quad 2 . \quad$ Bartolozzi, I.; Rizzi, F.; Frey, M., Comparison between hydrogen and electric vehicles by life Energy, Water and Environment Systems, 2013; Vol. 101, pp 103-111.

3. McKensey and Company A portfolio of power-trains for Europe: a fact-based analysis; 2010, 2010.

4. European Commission EU Energy, Transport and GHG Emissions. Trends to 2050.; Luxembourg, 2014.

5. ARF/M\&C Evolution. Electric vehicles in Europe: gearing up for a new phase?; The Netherlands, 2014.

6. Eurostat Statistics on the production of manufactured goods, 2015.

[http://epp.eurostat.ec.europa.eu/portal/page/portal/prodcom/data/database]

7. Joshi, S., Product Environmental Life-Cycle Assessment Using Input-Output Techniques. Journal of Industrial Ecology 1999, 3, (2-3), 95-120.

8. IRS Yearly Average Currency Exchange Rates. Translating foreign currency into U.S. dollars, 2015. [http://www.irs.gov/Individuals/International-Taxpayers/Yearly-Average-Currency-ExchangeRates]

9. OICA Production statitics, 2015. [http://www.oica.net/category/production-statistics/] 10. ACEA Consolidated Registrations - By Country, 2015. [http://www.acea.be/statistics/tag/category/by-country-registrations] 11. Tukker, A.; Huppes, G.; Guinée, J.; de Koning, A.; van Oers, L.; Suh, S.; Geerken, T.; Van Holderbeke, M.; Jensen, B.; Nielsen, P. Environmental Impact of Products (EIPRO). Analysis of the life cycle environmental impacts related to the final consumption of the EU-25; 2006.

12. Huppes, G.; de Koning, A.; Suh, S.; Heijungs, R.; van Oers, L.; Nielsen, P.; Guinée, J. B., Environmental Impacts of Consumption in the European Union:High-Resolution Input-Output Tables with Detailed Environmental Extensions. Journal of Industrial Ecology 2006, 10, (3), 129-146.

13. Suh, S., Developing a sectoral environmental database for input-output analysis: the comprehensive environmental data archive of the US. Economic Systems Research 2005, 17, (4), 449469.

14. Huppes, G.; de Koning, A.; Guinée, J.; Heijungs, R.; van Oers, L.; CML, R. K. Environmental Impacts of Diet Changes in the EU Annex: Description of E3IOT; 2009.

15. Tukker, A.; Goldbohm, R. A.; de Koning, A.; Verheijden, M.; Kleijn, R.; Wolf, O.; PérezDomínguez, I.; Rueda-Cantuche, J. M., Environmental impacts of changes to healthier diets in Europe. Ecological Economics 2011, 70, (10), 1776-1788.

16. Tukker, A.; de Koning, A.; Wood, R.; Hawkins, T.; Lutter, S.; Acosta, J.; Rueda Cantuche, J. M.; Bouwmeester, M.; Oosterhaven, J.; Drosdowski, T.; Kuenen, J., EXIOPOL - Development and illustrative analyses of a detailed global MR EE SUT/IOT. Economic Systems Research 2013, 25, (1), 50-70.

17. Wolf, O.; Pérez-Domínguez, I.; Rueda-Cantuche, J. M.; Tukker, A.; Kleijn, R.; de Koning, A.; Bausch-Goldbohm, S.; Verheijden, M., Do healthy diets in Europe matter to the environment? A quantitative analysis. Journal of Policy Modeling 2011, 33, (1), 8-28.

18. Genty, A.; Arto, I.; Neuwahl, F. Final database of environmental satellite accounts: technical report on their compilation; 2012.

19. Timmer, M. P.; Dietzenbacher, E.; Los, B.; Stehrer, R.; de Vries, G. J., An Illustrated User Guide to the World Input-Output Database: the Case of Global Automotive Production. Review of International Economics 2015, 23, (3), 575-605.

20. Koesler, S.; Pothen, F. The Basic WIOD CGE Model: A computable general equilibrium model based on the World Input-Output Database; ZEW-Dokumentation: 2013. 
21. Boitier, B. CO2 emissions production-based accounting vs consumption: Insights from the WIOD databases, WIOD Conference Paper, 2012.

278 22. Wood, R.; Stadler, K.; Bulavskaya, T.; Lutter, S.; Giljum, S.; de Koning, A.; Kuenen, J.; Schütz, 279 H.; Acosta-Fernández, J.; Usubiaga, A.; Simas, M.; Ivanova, O.; Weinzettel, J.; Schmidt, J.; Merciai, S.;

280 Tukker, A., Global Sustainability Accounting-Developing EXIOBASE for Multi-Regional Footprint 281 Analysis. Sustainability 2014, 7, (1), 138-163.

282 23. Tukker, A.; Bulavskaya, T.; Giljum, S.; de Koning, A.; Lutter, S.; Simas, M.; Stadler, K.; Wood, 283 R., The Global Resource Footprint of Nations-Carbon, Water, Land and Materials Embodied in Trade and Final Consumption Calculated with Exiobase 2.1. In The Netherlands Organisation for Applied Scientific Research, 2014.

24. Schoer, K.; Wood, R.; Arto, I.; Weinzettel, J., Estimating Raw Material Equivalents on a MacroLevel: Comparison of Multi-Regional Input-Output Analysis and Hybrid LCl-IO. Environmental Science \& Technology 2013, 47, (24), 14282-14289. 